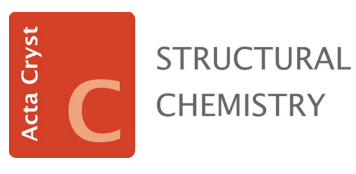

ISSN 2053-2296

Received 11 June 2020

Accepted 23 June 2020

Edited by A. L. Spek, Utrecht University, The Netherlands

Keywords: celecoxib; active pharmaceutical ingredient; API; solvate; crystal structure; isostructurality; disorder; PIXEL; anti-inflammatory.

CCDC references: 2011633; 2011634; 2011635; 2011636; 2011637; 2011638

Supporting information: this article has supporting information at journals.iucr.org/C

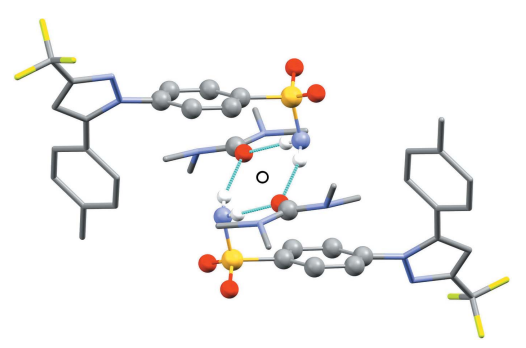

\section{Intermolecular interactions and disorder in six isostructural celecoxib solvates}

\author{
Andrew D. Bond ${ }^{\mathrm{a}, \mathrm{b} *}$ and Changquan C. $\operatorname{Sun}^{\mathrm{c}}$

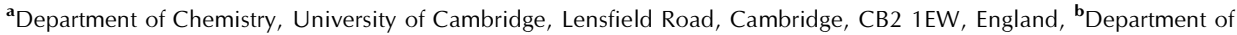 \\ Pharmacy, University of Copenhagen, Universitetsparken 2, Copenhagen, DK-2100, Denmark, and ${ }^{\mathrm{C}}$ Department of \\ Pharmaceutics, University of Minnesota, Minneapolis, MN 5545, USA. *Correspondence e-mail: adb29@cam.ac.uk
}

Six isostructural crystalline solvates of the active pharmaceutical ingredient celecoxib \{4-[5-(4-methylphenyl)-3-(trifluoromethyl)pyrazol-1-yl]benzenesulfonamide; $\mathrm{C}_{17} \mathrm{H}_{14} \mathrm{~F}_{3} \mathrm{~N}_{3} \mathrm{O}_{2} \mathrm{~S}$ \} are described, containing dimethylformamide (DMF, $\mathrm{C}_{3} \mathrm{H}_{7} \mathrm{NO}, 1$ ), dimethylacetamide (DMA, $\mathrm{C}_{4} \mathrm{H}_{9} \mathrm{NO}, 2$ ), $\mathrm{N}$-methylpyrrolidin-2-one (NMP, $\mathrm{C}_{5} \mathrm{H}_{9} \mathrm{NO}, 3$ ), tetramethylurea (TMU, $\mathrm{C}_{5} \mathrm{H}_{12} \mathrm{~N}_{2} \mathrm{O}, 4$ ), 1,3-dimethyl-3,4,5,6tetrahydropyrimidin-2(1H)-one (DMPU, $\mathrm{C}_{6} \mathrm{H}_{12} \mathrm{~N}_{2} \mathrm{O}, 5$ ) or dimethyl sulfoxide (DMSO, $\mathrm{C}_{2} \mathrm{H}_{6} \mathrm{OS}, \mathbf{6}$ ). The host celecoxib structure contains one-dimensional channel voids accommodating the solvent molecules, which accept hydrogen bonds from the $\mathrm{NH}_{2}$ groups of two celecoxib molecules. The solvent binding sites have local twofold rotation symmetry, which is consistent with the point symmetry of the solvent molecule in $\mathbf{4}$ and $\mathbf{5}$, but introduces orientational disorder for the solvent molecules in $\mathbf{1}, \mathbf{2}, \mathbf{3}$ and $\mathbf{6}$. Despite the isostructurality of 1-6, the unit-cell volume and solvent-accessible void space show significant variation. In particular, $\mathbf{4}$ and $\mathbf{5}$ show an enlarged and skewed unit cell, which can be attributed to a specific interaction between an $\mathrm{N}-\mathrm{CH}_{3}$ group in the solvent molecule and the toluene group of celecoxib. Intermolecular interaction energies calculated using the PIXEL method show that the total interaction energy between the celecoxib and solvent molecules is broadly correlated with the molecular volume of the solvent, except in $\mathbf{6}$, where the increased polarity of the $\mathrm{S}=\mathrm{O}$ bond leads to greater overall stabilization compared to the similarlysized DMF molecule in $\mathbf{1}$. In the structures showing disorder, the most stable orientations of the solvent molecules make $\mathrm{C}-\mathrm{H} \cdots \mathrm{O}$ contacts to the $\mathrm{S}=\mathrm{O}$ groups of celecoxib.

\section{Introduction}

Understanding the structures and properties of crystalline solids can be of significant importance for active pharmaceutical ingredients (APIs) (Sun, 2009). Solid-form screening is an integral part of most pre-formulation activities (Morissette et al., 2004), with an aim to establish the range of solid forms that can exist for a given API. These generally include both polymorphs and multicomponent forms, which may variously be described as salts, cocrystals, solvates, etc. (Aitipamula et al., 2012).

The API of interest in this work is the anti-inflammatory drug celecoxib (see Scheme 1). To date, there is only one polymorph (Form III) of celecoxib for which a single-crystal X-ray structure has been reported (Dev et al., 1999; Wang et al. , 2019) in the Cambridge Structural Database (CSD; Groom et al., 2016), although several polymorphs are established in the literature (Dev et al., 1999; Lu et al., 2006; Wang \& Sun, 2019). Crystal structures are known for numerous multicomponent forms, including with pyrrolidin-2-one, caprolactam, valerolactam (Bolla et al., 2014), pyridin-2(1H)-one (Bolla \& Nangia, 2019), nicotinamide (Zhang et al., 2017) and bis(proline) (as a zwitterion; Li et al., 2018). Some celecoxib 
Table 1

Experimental details.

For all structures: monoclinic, $P 2_{1} / c, Z=4$. Experiments were carried out at $298 \mathrm{~K}$ with $\mathrm{Cu} K \alpha$ radiation using a Bruker D8-QUEST PHOTON-100 diffractometer. Absorption was corrected for by multi-scan methods ( $S A D A B S$; Bruker, 2016). $\mathrm{H}$ atoms were treated by a mixture of independent and constrained refinement.

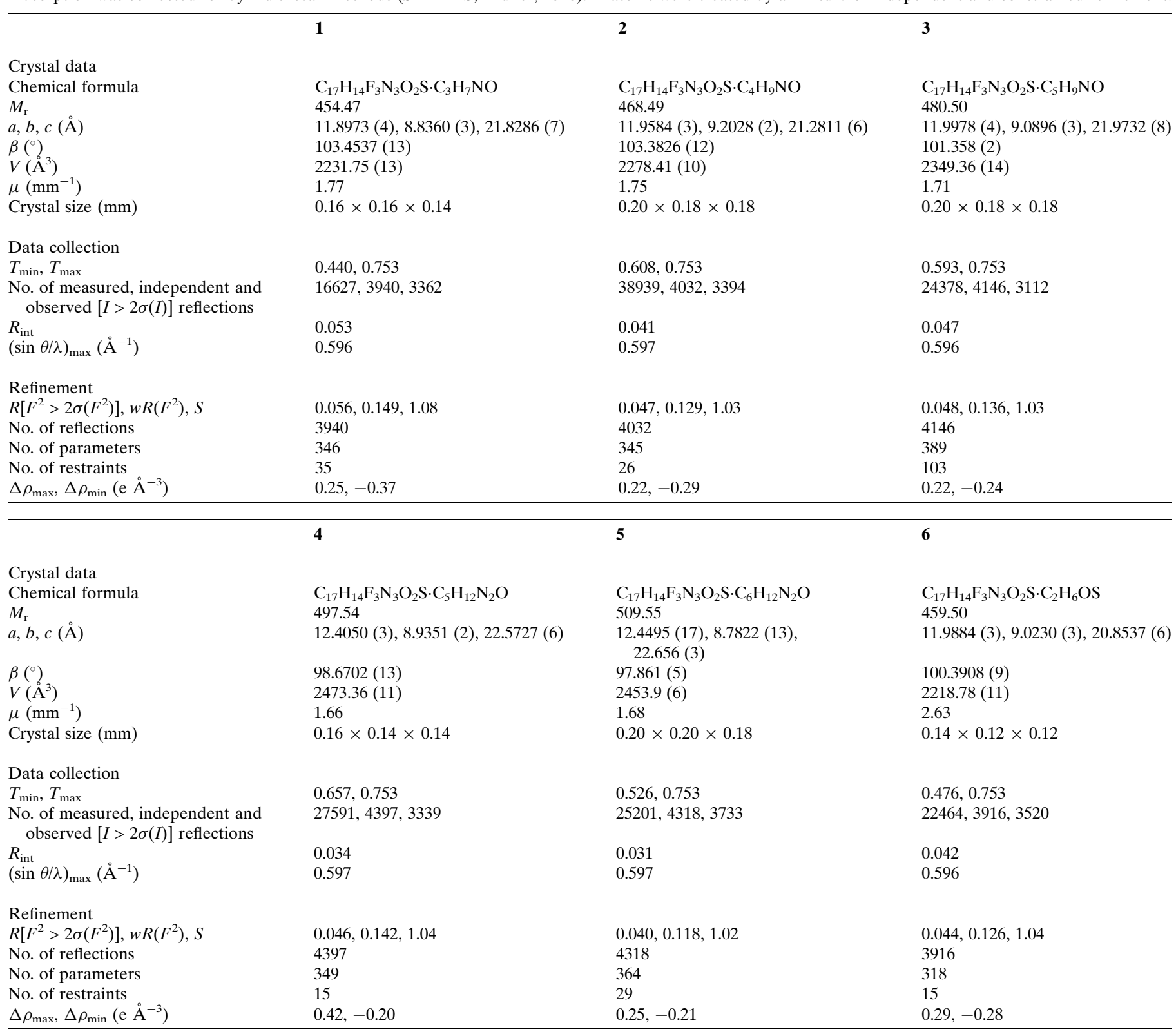

Computer programs: APEX3 (Bruker, 2018), SAINT (Bruker, 2018), SHELXT (Sheldrick, 2015a), SHELXL2018 (Sheldrick, 2015b) and Mercury (Macrae et al., 2020).

cocrystals with 4,4'-bipyridine and 1,2-bis(pyridin-4-yl)ethylene have also been reported to form isostructural solvates (i.e. isostructural three-component crystals) when combined with acetone, THF or 1,4-dioxane (Wang et al., 2014).

We were initially interested in studying solvates of celecoxib as part of a broader structure-property correlation exercise to understand and address its pharmaceutical deficiencies, e.g. high punch sticking propensity (Wang et al., 2020; Paul et al., 2017, 2020), amorphous phase stability (Wang \& Sun, 2019), poor flowability (Chen et al., 2020) and high elastic flexibility (Wang et al., 2019). In the course of this work, we identified a group of six solvates (see Scheme 1) that form an isostructural group, different from any of the multicomponent celecoxib crystal structures in the CSD. We describe the new structure type in this paper and explore aspects of the isostructurality, including variation of the unit-cell parameters, disorder of the solvent molecules, and the intermolecular interactions between the solvent and celecoxib molecules.

\section{Experimental}

\subsection{Synthesis and crystallization}

Single crystals suitable for X-ray analysis were obtained by slow cooling of a warm solution of celecoxib in either di- 
methylformamide (DMF, 1), dimethylacetamide (DMA, 2), $N$-methylpyrrolidin-2-one (NMP, 3), tetramethylurea (TMU, 4), 1,3-dimethyl-3,4,5,6-tetrahydropyrimidin-2(1H)-one (DMPU, 5) or dimethyl sulfoxide (DMSO, 6). The DMF (1) and DMA (2) solvates have been prepared previously (Chawla et al., 2003), but structural details were not provided. A powder X-ray diffraction pattern published by Chawla et al. (2003) clearly matches that simulated from the crystal structure of 2 . The match to $\mathbf{1}$ is less clear, but additional thermal analysis is broadly consistent with our observations, so it seems probable that the structures described herein are consistent with the previously studied material.<smiles>CC(=O)N(C)C(=O)N(C)C(=O)N(C)C(=O)N(C)C(=O)N(C)C</smiles>

\subsection{Refinement of $1-6$}

$\mathrm{H}$ atoms bound to $\mathrm{C}$ atoms were placed in idealized positions and refined using a riding model, with $U_{\text {iso }}(\mathrm{H})=1.2$ or $1.5 U_{\text {eq }}(\mathrm{C})$. For the methyl group $(\mathrm{C} 11)$ in celecoxib, the $\mathrm{H}$ atoms were allowed to rotate around the local threefold axis. $\mathrm{H}$ atoms of the $\mathrm{NH}_{2}$ groups were located in difference Fourier maps, then refined with isotropic displacement parameters, with the $\mathrm{N}-\mathrm{H}$ and $\mathrm{H} \cdots \mathrm{H}$ distances restrained to 0.86 (1) and 1.50 (1) $\AA$, respectively. All of the structures display rotational disorder of the $\mathrm{CF}_{3}$ group. This was modelled in each case as two sets of three $\mathrm{F}$ atoms, with site-occupancy factors constrained to sum to unity. To ensure a regular geometry, the $\mathrm{C}-\mathrm{F}$ distances were restrained to a common refined value and the F ...F distances were restrained to 1.633 times that value. All $\mathrm{F}$ atoms were refined with anisotropic ADPs. This produces highly distorted (prolate) ellipsoids in several cases, despite the inclusion of two sets of atomic sites, indicating that the rotational disorder is extensive. Given this rotational

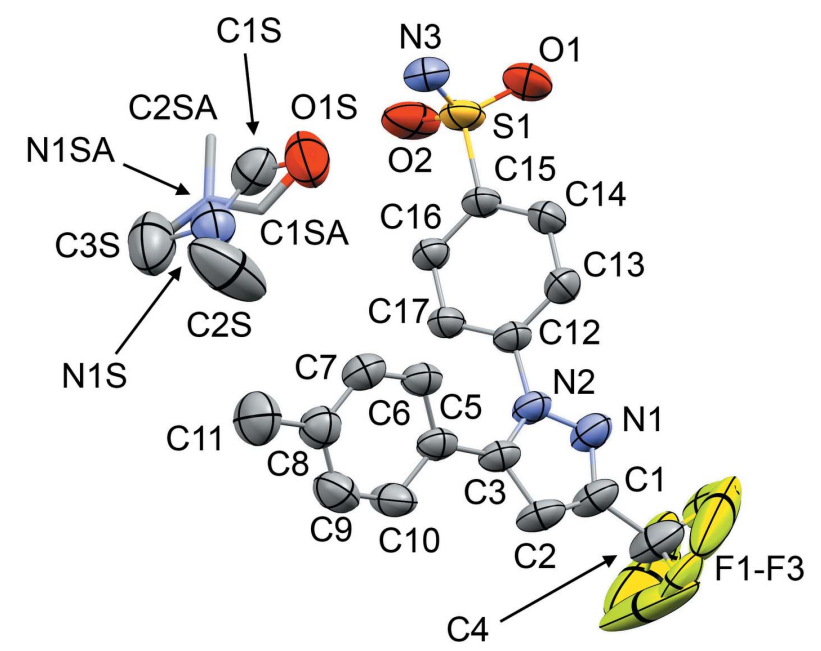

Figure 1

The molecular structure of $\mathbf{1}$, with displacement ellipsoids at the $50 \%$ probability level. $\mathrm{H}$ atoms have been omitted. The second disorder component of the DMF molecule [site-occupancy factor $=0.221(7)$ ] is shown in outline only. Atoms $\mathrm{O} 1 S$ and $\mathrm{C} 3 S$ are common to both DMF components.

disorder, the distorted ellipsoids were considered to be an acceptable compromise to model the electron density in this region. For the disordered solvent molecules in 1, 2, 3 and $\mathbf{6}$, two sets of atoms were refined, with site-occupancy factors summing to unity, and with appropriate geometrical restraints. Anisotropic ADPs were applied to all non- $\mathrm{H}$ atoms and $\mathrm{H}$ atoms were placed in idealized positions and refined as riding. The structure and refinement details are presented in Table 1.

\subsection{Computational details}

The crystal structures were energy-minimized with dispersion-corrected density functional theory (DFT-D) using the CASTEP module (Clark et al., 2005) in Materials Studio (Accelrys, 2011). The PBE functional (Perdew et al., 1996) was applied with a plane-wave cut-off energy of $520 \mathrm{eV}$, in combination with the Grimme semi-empirical dispersion correction (Grimme, 2006). The unit-cell parameters were constrained to the experimental values and the space group $P 2_{1} / c$ was imposed. Only one component of the rotationallydisordered $\mathrm{CF}_{3}$ group was included (arbitrarily). For the structures with disordered solvent molecules, separate models were minimized for each disorder component. In each case, minimization produced only small geometrical deviations from the starting structure, as expected for high-quality singlecrystal structures (van de Streek \& Neumann, 2010). The purpose of the optimization step is to place the structures on a common basis for comparison, particularly for the disordered structures, where the results of the crystallographic refinement are generally less precise. The DFT-D-optimized structures were then used as input for the PIXEL module of the CSP package (Gavezzotti, 2002, 2003, 2011). The calculated pairwise interaction energies are estimated to have an accuracy within a range of $c a \pm 3 \mathrm{~kJ} \mathrm{~mol}^{-1}$. 


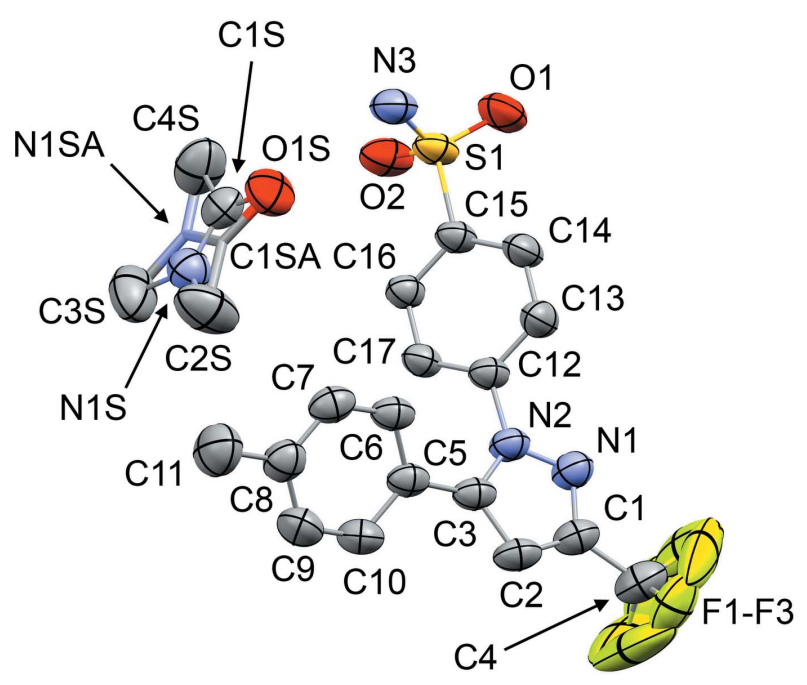

Figure 2

The molecular structure of $\mathbf{2}$, with displacement ellipsoids at the $50 \%$ probability level. $\mathrm{H}$ atoms have been omitted. The second disorder component of the DMA molecule [site-occupancy factor $=0.464(8)$ ] is shown in outline only. Atoms $\mathrm{O} 1 S, \mathrm{C} 2 S, \mathrm{C} 3 S$ and $\mathrm{C} 4 S$ are common to both DMA components.

\section{Results and discussion}

\subsection{Description of the crystal structures}

The molecular structures of 1-6 are shown in Figs. 1-6. In the crystal structures, the celecoxib molecules are arranged into pairs around inversion centres with two solvent molecules accepting $\mathrm{N}-\mathrm{H} \cdots \mathrm{O}$ hydrogen bonds (Fig. 7). The local symmetry of this unit is effectively $2 / m\left(C_{2 h}\right)$, where the $\mathrm{C}=\mathrm{O}$ (or $\mathrm{S}=\mathrm{O}$ ) group of each solvent molecule is approximately aligned along the local twofold rotation axis. For the TMU and DMPU molecules in $\mathbf{4}$ and $\mathbf{5}$, which themselves show twofold rotational point symmetry, this produces an ordered crystal-

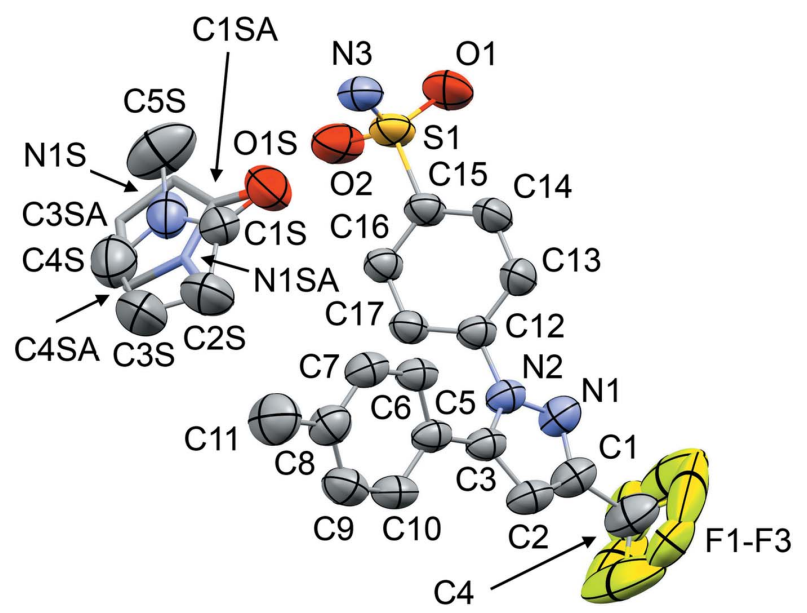

Figure 3

The molecular structure of $\mathbf{3}$, with displacement ellipsoids at the $50 \%$ probability level. $\mathrm{H}$ atoms have been omitted. The second disorder component of the NMP molecule [site-occupancy factor $=0.321(8)$ ] is shown in outline only. Atoms C2SA and C5SA are not labelled, C2SA is directly below $\mathrm{C} 5 S$ and $\mathrm{C} 5 S A$ is hidden behind $\mathrm{C} 2 S$.

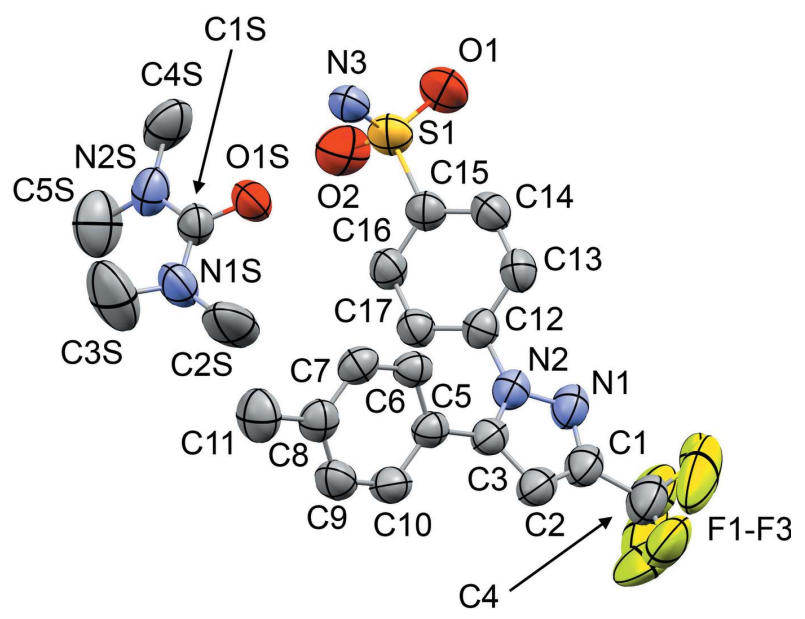

Figure 4

The molecular structure of $\mathbf{4}$, with displacement ellipsoids at the $50 \%$ probability level. $\mathrm{H}$ atoms have been omitted. Disorder is not evident for the TMU molecule.

lographic result. The DMPU molecule displays minor conformational disorder of its six-membered ring (atoms C3S/ C3SA in Fig. 5), but the parts of the molecule involved in binding to celecoxib are ordered and consistent for TMU and DMPU. For DMF (1), DMA (2), NMP (3) and DMSO (6), which do not possess twofold rotational symmetry, the crystal structure is disordered, with the molecules adopting two alternative orientations related by the local twofold axis. However, the full crystallographic environment of each solvent molecule is not twofold symmetric. Hence, the two orientations of the disordered solvent molecules have different total interaction energies (\$3.3). With the solvent molecules removed from the structures, the void space between the celecoxib molecules defines one-dimensional (1D) channels along the $b$ axis (Fig. 8). The solvent-accessible volume spans a considerable range for 1-6 (Table 2), constituting approximately $21-28 \%$ of the unit-cell volume.

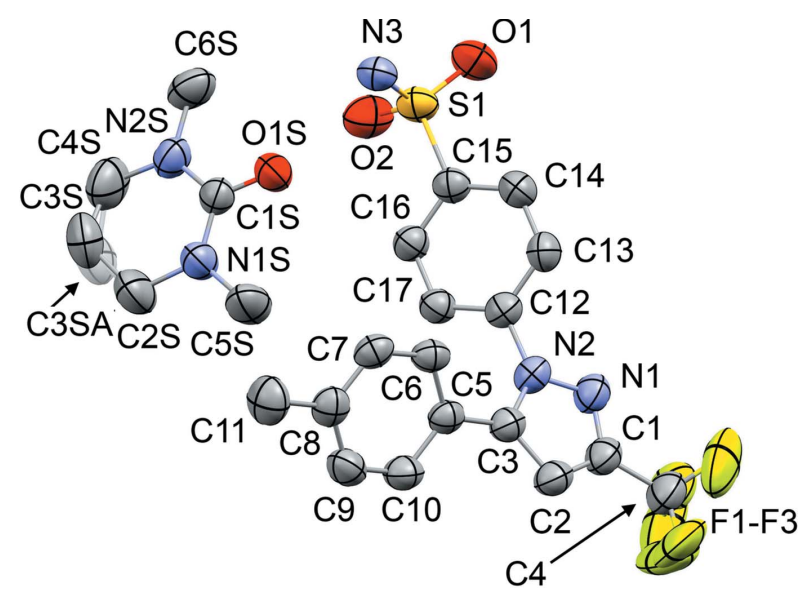

Figure 5

The molecular structure of $\mathbf{5}$, with displacement ellipsoids at the $50 \%$ probability level. $\mathrm{H}$ atoms have been omitted. The alternative positions C3S and C3SA [site-occupancy factors $=0.584$ (16):0.416 (16)] are shown for the DMPU molecule. 


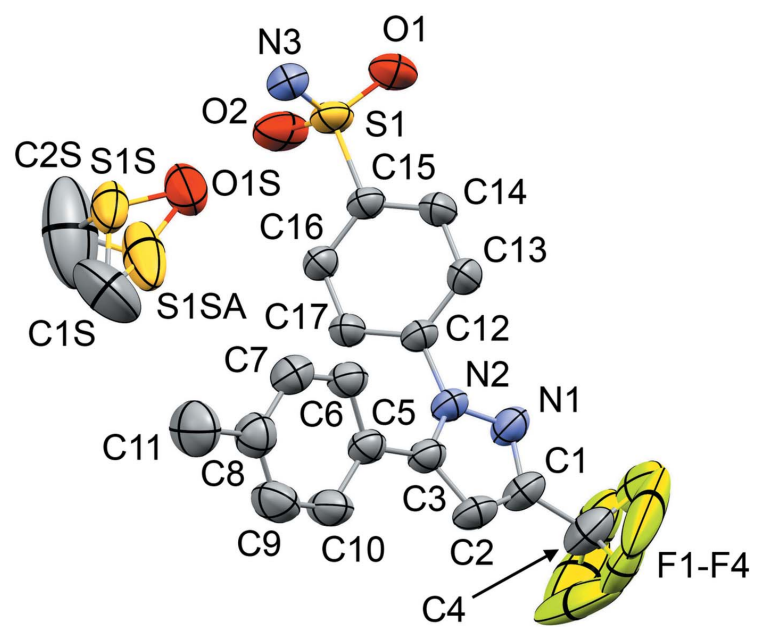

Figure 6

The molecular structure of $\mathbf{6}$, with displacement ellipsoids at the $50 \%$ probability level. $\mathrm{H}$ atoms have been omitted. Atoms $\mathrm{C} 1 S$ and $\mathrm{C} 2 S$ are common to both disorder components for the DMSO molecule. The relatively large displacement ellipsoid of atom $\mathrm{C} 2 S$ was retained in preference to multiple atom sites for simplicity of the model.

\subsection{Variation of the unit-cell parameters}

Despite the isostructural nature of the solvates, the unit-cell parameters differ quite significantly, with a difference of $c a$ $250 \AA^{3}$ between the smallest (6) and largest (4) volumes. Plotting the $b$ or $c$ axis of 1-6 by ascending length (see supporting information) shows an approximately linear change in each case, but plotting the $a$ axis in a similar manner shows a clear discontinuity, with the $a$ axis in $\mathbf{4}$ and $\mathbf{5}$ being approximately $0.5 \AA$ longer than in $\mathbf{1}, \mathbf{2}, \mathbf{3}$ and $\mathbf{6}$. A similar pattern is seen for the $\beta$ angle, indicating a relative skewing of the $a c$ plane in $\mathbf{4}$ and $\mathbf{5}$. Comparing representative structures in projection along the $b$ axis (Fig. 9) indicates a reason for this observation. Common to structures $\mathbf{4}$ and $\mathbf{5}$, but not present in

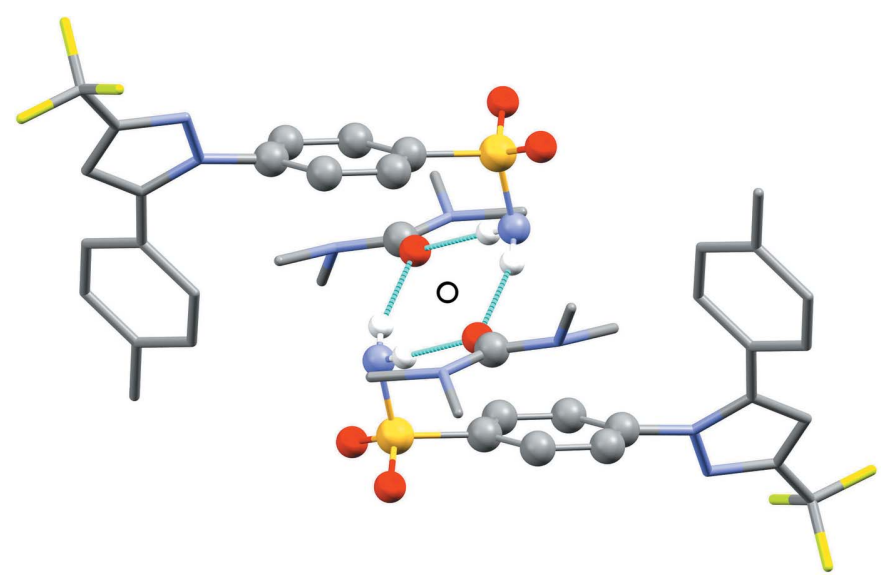

Figure 7

Hydrogen-bonded motif with two solvent molecules (TMU is shown) accepting $\mathrm{N}-\mathrm{H} \cdots \mathrm{O}$ hydrogen bonds from two celecoxib molecules across a crystallographic inversion centre (indicated by the open circle). $\mathrm{H}$ atoms not involved in hydrogen bonding have been omitted. The atoms shown in ball-and-stick style conform to local $2 / m\left(C_{2 \mathrm{~h}}\right)$ point symmetry.
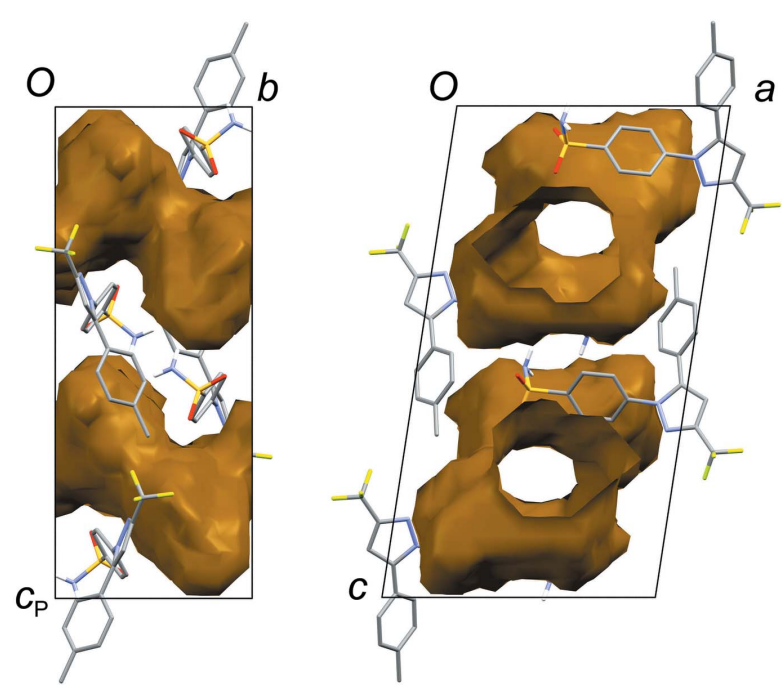

Figure 8

Views of the $1 \mathrm{D}$ voids running along the $b$ axis in the celecoxib framework structure, after removing the solvent molecules. Structure $\mathbf{6}$ is shown. The voids are generated using Mercury (contact surface, probe radius $1.2 \AA$; Macrae et al., 2020).

$\mathbf{1}, \mathbf{2}, \mathbf{3}$ or $\mathbf{6}$, is an $\mathrm{N}-\mathrm{CH}_{3}$ group in the solvent molecule that points approximately along the $a$ axis and is directed towards a neighbouring toluene ring of celecoxib. The interaction pushes the toluene ring away from the position seen in the structures that do not have this $\mathrm{N}-\mathrm{CH}_{3}$ group. The celecoxib molecules are 'anchored' by their hydrogen-bonding $\mathrm{NH}_{2}$ groups, which retain essentially identical positions in all structures, so the effect of pushing away the toluene ring is a relative rotation of the celecoxib molecules (Fig. 9). This serves to elongate both the $a$ and the $c$ axes, and to skew the unit cell. The other $\mathrm{CH}_{3}$ groups in TMU (4) or DMPU (5) adopt positions that are seen in one or more of the other structures, and they do not make any clearly comparable intermolecular contacts to celecoxib.

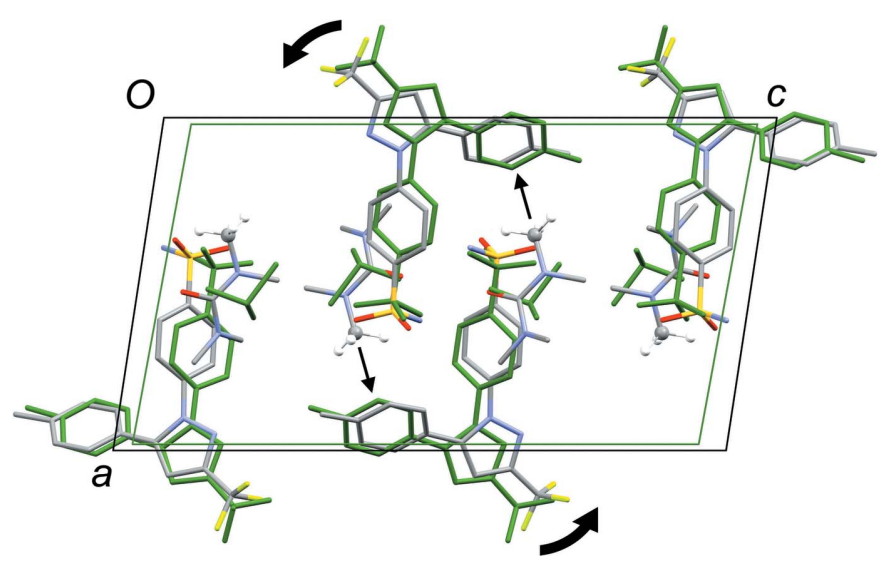

Figure 9

Projection of the structures of $\mathbf{4}$ (standard colour) and $\mathbf{6}$ (green) along the $b$ axis. The highlighted $\mathrm{N}-\mathrm{CH}_{3} \cdots \pi$ interaction in $4[\mathrm{C} 2 S \cdots$ centroid(C5C10) $=3.825 \AA]$ causes the celecoxib molecules to rotate outwards relative to each other, as indicated by the thick arrows, causing expansion and skewing of the unit cell compared to 6. 
Table 2

Intermolecular interaction energies between the celecoxib and solvent molecules $\left[E(\text { tot })_{\text {cel-solv }}\right]$ and between the solvent molecules $\left[E(\text { tot })_{\text {solv-solv }}\right]$, calculated using the PIXEL method, together with the unit-cell volume $\left(V_{\text {cell }}\right)$, void volume $\left(V_{\text {void }}\right)$ and solvent molecular volume $\left(V_{\text {solv }}\right)$.

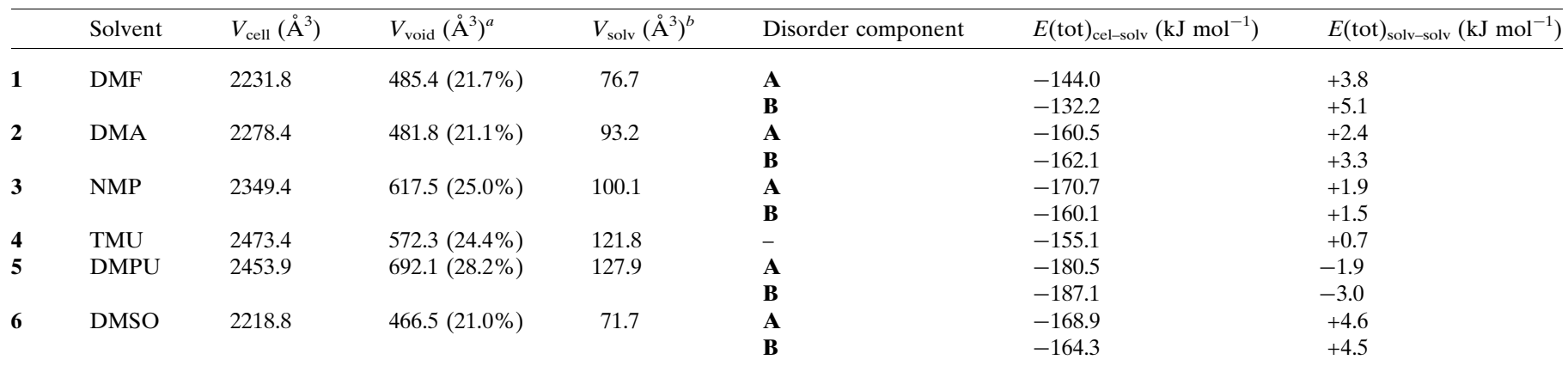

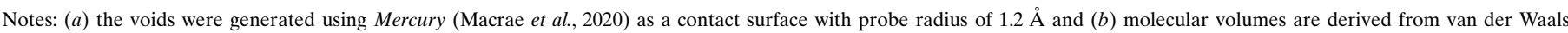
surfaces, calculated in Materials Studio (Accelrys, 2011) as a Connolly surface generated with zero probe radius.

\subsection{Interactions between the solvent molecules and cele-} coxib

On account of the isostructurality, the pairwise intermolecular interactions in each structure can be directly matched. Table 2 lists the total interaction energy between the celecoxib and solvent molecules, based on an equivalent set of interactions in each structure. For 1-5, the total celecoxibsolvent interaction energy broadly increases with the molecular volume of the solvent, with 2 (DMA) and 3 (NMP) being closely comparable. The DMSO molecule in $\mathbf{6}$ has a significantly more stabilizing total interaction with celecoxib, compared to the similarly-sized DMF molecule in $\mathbf{1}$, due to the increased polarity of the $\mathrm{S}=\mathrm{O}$ bond. For example, the two independent pairwise interactions including the hydrogen bonds to celecoxib are both approximately $-50 \mathrm{~kJ} \mathrm{~mol}^{-1}$ in $\mathbf{1}$ (varying slightly for the two disorder components), but approximately -57 and $-68 \mathrm{~kJ} \mathrm{~mol}^{-1}$ in $\mathbf{6}$. The $\mathrm{N}-\mathrm{CH}_{3} \cdots \pi$ interaction highlighted in Fig. 9 belongs to the celecoxibsolvent pair within the asymmetric unit (as shown in Figs. 4 and 5). Since PIXEL energies refer to total pairwise intermolecular interactions, any specific features of the $\mathrm{N}-$ $\mathrm{CH}_{3} \cdots \pi$ interaction are masked by the total interaction energy. Table 2 also lists the total interaction energy between solvent molecules, based on three equivalent significant

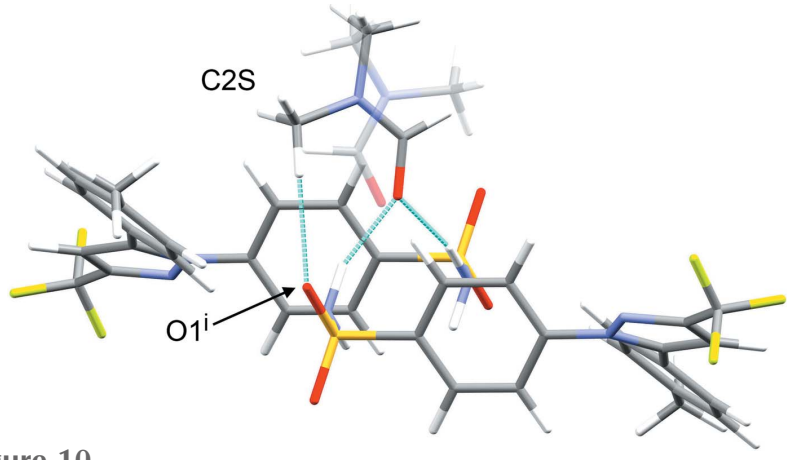

Figure 10

The most stable orientation of the disordered DMF molecule in $\mathbf{1}$, highlighting the $\mathrm{C}-\mathrm{H} \cdots \mathrm{O}$ contact to an $\mathrm{S}=\mathrm{O}$ group of celecoxib [symmetry code: (i) $-x+1,-y+1,-z+1]$. The less stable DMF orientation is shown as semi-transparent. interactions in each structure. The interaction between the two solvent molecules involved in the hydrogen-bonded motif (Fig. 7) is repulsive, due to the destabilizing Coulombic $\mathrm{O} \cdots \mathrm{O}$ interaction. The only other significant interactions between solvent molecules are along the $2_{1}$ screw axis parallel to $b$, which are slightly stabilizing. The extent to which these interactions mitigate the destabilizing $\mathrm{O} \cdots \mathrm{O}$ interaction increases with the molecular volume of the solvent, and the overall solvent-solvent interaction is slightly stabilizing for the largest molecule, i.e. DMPU (5).

The difference between the total interaction energies with the celecoxib framework for the two disorder components of the solvent molecules in each structure is also shown in Table 2. The most significant difference within a single structure is seen for DMF (1), where the two orientations exchange the positions of the $\mathrm{C}-\mathrm{H}$ and $\mathrm{N}-\mathrm{CH}_{3}$ groups (Fig. 1). Approximately two thirds of the energy difference arises from the interactions of the DMF molecule with the two celecoxib molecules in the hydrogen-bonding motif (Fig. 7), where the more stable DMF

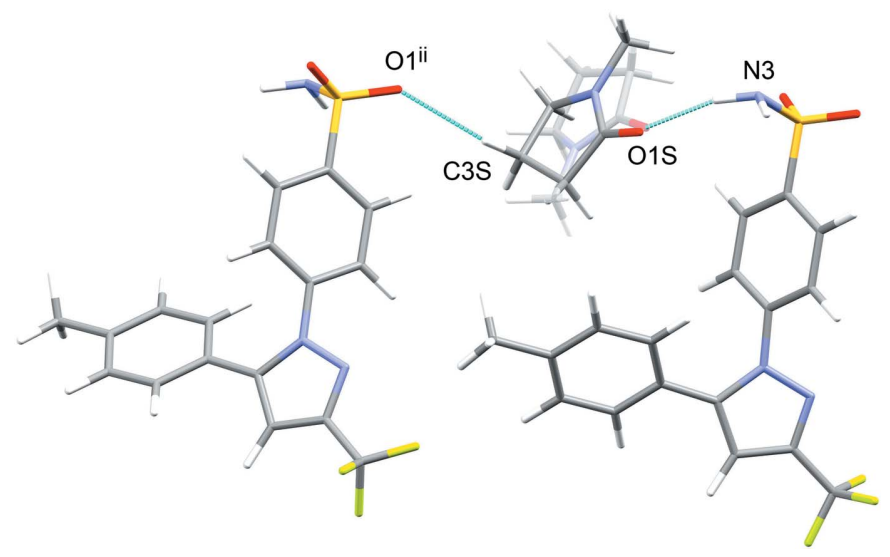

Figure 11

The most stable orientation of the disordered NMP molecule in $\mathbf{3}$. A short $\mathrm{C}-\mathrm{H} \cdots \mathrm{O}$ contact is made to an $\mathrm{S}=\mathrm{O}$ group of the neighbouring celecoxib molecule [symmetry code: (ii) $x,-y+\frac{3}{2}, z-\frac{1}{2}$ ]. The less stable NMP orientation for 3 is shown as semi-transparent. A comparable C$\mathrm{H}$... O contact is seen for the most stable disorder component of DMPU in 5. 
orientation brings the $\mathrm{CH}_{3}$ group of atom $\mathrm{C} 2 \mathrm{~S}$ into close proximity to $\mathrm{O} 1$ in one of the $\mathrm{S}=\mathrm{O}$ bonds (Fig. 10). For DMA (2), the disorder components have essentially the same molecular footprint (the positions of atoms $\mathrm{O} 1 S, \mathrm{C} 2 S, \mathrm{C} 3 S$ and $\mathrm{C} 4 S$ are common to both DMA components; Fig. 2), and the total interaction energies of the two components with the celecoxib framework are the same within the expected precision of the calculations. For NMP (3), the two solvent orientations are geometrically comparable, except for the positions of $\mathrm{C} 3 \mathrm{~S} / \mathrm{C} 3 \mathrm{SA}$ (Fig. 3). However, one orientation is noticeably more stable than the other. A significant energy difference also exists for the two orientations of the DMPU molecule in $\mathbf{5}$, for which the principal geometrical difference is the position of one $\mathrm{CH}_{2}$ group $(\mathrm{C} 3 S / \mathrm{C} 3 S A)$, with accompanying differences in the positions of the $\mathrm{H}$ atoms on the neighbouring $\mathrm{CH}_{2}$ groups. Comparing the most stable disorder components for $\mathbf{3}$ and $\mathbf{5}$, they share a common position for one $\mathrm{CH}_{2}$ group ( $\mathrm{C} 3 \mathrm{~S}$ in $\mathbf{3}$ and $\mathrm{C} 2 \mathrm{~S}$ in $\left.\mathbf{5}\right)$ that is not seen in the other disorder components. This introduces a short $\mathrm{C}-\mathrm{H} \cdots \mathrm{O}$ contact to an $\mathrm{S}=\mathrm{O}$ group of the neighbouring celecoxib molecule (Fig. 11). The PIXEL energies confirm that the interaction with this celecoxib molecule is significantly more stabilizing when this contact is present than when it is not. For the DMSO molecules in 6, the difference between the two solvent orientations involves only the position of the $\mathrm{S}$ atom, and the interaction energies with the celecoxib framework are comparable.

\section{Conclusion}

This set of six isostructural celecoxib solvates includes small solvent molecules that can accept hydrogen bonds. The host celecoxib framework is consistent within the set, but it shows quite substantial flexibility in its unit-cell parameters and solvent-accessible void space, and can therefore accommodate solvent molecules of varying size and shape. The crystallographic disorder in several of the structures is understandable on the basis of the local twofold symmetry of the solvent binding site, compared to the point symmetry of the solvent molecules. In the absence of any additional hydrogenbond donors in the solvent molecules, the next most stabilizing interactions between the solvent molecules and the celecoxib framework are $\mathrm{C}-\mathrm{H} \cdots \mathrm{O}$ contacts to the $\mathrm{S}=\mathrm{O}$ groups. The consideration of PIXEL interaction energies, in combination with geometrical analysis of the crystal structures, is helpful in drawing these conclusions.

\section{Funding information}

Funding for this research was provided by: The Lundbeck Foundation (Denmark) (grant No. R143-2014-25, Visiting
Professorship to CCS); Danish Council for Independent Research | Natural Sciences (grant No. DFF-1323-00122); Department of Pharmacy, University of Copenhagen.

\section{References}

Accelrys (2011). Materials Studio. Accelrys Software Inc., San Diego, CA, USA.

Aitipamula, S., et al. (2012). Cryst. Growth Des. 12, 2147-2152.

Bolla, G., Mittapalli, S. \& Nangia, A. (2014). CrystEngComm, 16, 24 27.

Bolla, G. \& Nangia, A. (2019). IUCrJ, 6, 751-760.

Bruker (2016). SADABS. Bruker AXS Inc., Madison, Wisconsin, USA.

Bruker (2018). APEX3 and SAINT. Bruker AXS Inc., Madison, Wisconsin, USA.

Chawla, G., Gupta, P., Thilagavathi, R., Chakraborti, A. K. \& Bansal, A. K. (2003). Eur. J. Pharm. Sci. 20, 305-317.

Chen, H., Paul, S., Xu, H., Wang, K., Mahanthappa, M. K. \& Sun, C. C. (2020). Mol. Pharm. 17, 1387-1396.

Clark, S. J., Segall, M. D., Pickard, C. J., Hasnip, P. J., Probert, M. J., Refson, K. \& Payne, M. C. (2005). Z. Kristallogr. 220, 567-570.

Dev, R. V., Rekha, K. S., Vyas, K., Mohanti, S. B., Kumar, P. R. \& Reddy, G. O. (1999). Acta Cryst. C55, 9900161.

Gavezzotti, A. (2002). J. Phys. Chem. B, 106, 4145-4154.

Gavezzotti, A. (2003). J. Phys. Chem. B, 107, 2344-2353.

Gavezzotti, A. (2011). New J. Chem. 35, 1360.

Grimme, S. (2006). J. Comput. Chem. 27, 1787-1799.

Groom, C. R., Bruno, I. J., Lightfoot, M. P. \& Ward, S. C. (2016). Acta Cryst. B72, 171-179.

Li, D., Kong, M., Li, J., Deng, Z. \& Zhang, H. (2018). CrystEngComm, 20, 5112-5118.

Lu, G. W., Hawley, M., Smith, M., Geiger, B. M. \& Pfund, W. (2006). J. Pharm. Sci. 95, 305-317.

Macrae, C. F., Sovago, I., Cottrell, S. J., Galek, P. T. A., McCabe, P., Pidcock, E., Platings, M., Shields, G. P., Stevens, J. S., Towler, M. \& Wood, P. A. (2020). J. Appl. Cryst. 53, 226-235.

Morissette, S. L., Almarsson, O., Peterson, M. L., Remenar, J. F., Read, M. J., Lemmo, A. V., Ellis, S., Cima, M. J. \& Gardner, C. R. (2004). Adv. Drug Deliv. Rev. 56, 275-300.

Paul, S., Taylor, L. J., Murphy, B., Krzyzaniak, J., Dawson, N., Mullarney, M. P., Meenan, P. \& Sun, C. C. (2017). J. Pharm. Sci. 106, 151-158.

Paul, S., Taylor, L. J., Murphy, B., Krzyzaniak, J. F., Dawson, N., Mullarney, M. P., Meenan, P. \& Sun, C. C. (2020). Mol. Pharm. 17, 1148-1158.

Perdew, J. P., Burke, K. \& Ernzerhof, M. (1996). Phys. Rev. Lett. 77, 3865-3868.

Sheldrick, G. M. (2015a). Acta Cryst. A71, 3-8.

Sheldrick, G. M. (2015b). Acta Cryst. C71, 3-8.

Streek, J. van de \& Neumann, M. A. (2010). Acta Cryst. B66, 544-558.

Sun, C. C. (2009). J. Pharm. Sci. 98, 1671-1687.

Wang, C., Paul, S., Sun, D. J., Nilsson Lill, S. O. \& Sun, C. C. (2020). Cryst. Growth Des. doi: 10.1021/acs.cgd.0c00492.

Wang, K., Mishra, M. K. \& Sun, C. C. (2019). Chem. Mater. 31, 17941799.

Wang, K. \& Sun, C. C. (2019). Cryst. Growth Des. 19, 3592-3600.

Wang, X., Zhang, Q., Jiang, L., Xu, Y. \& Mei, X. (2014). CrystEngComm, 16, 10959-10968.

Zhang, S.-W., Brunskill, A. P. J., Schwartz, E. \& Sun, S. (2017). Cryst. Growth Des. 17, 2836-2843. 


\section{supporting information}

Acta Cryst. (2020). C76, 632-638 [https://doi.org/10.1107/S2053229620008359]

\section{Intermolecular interactions and disorder in six isostructural celecoxib solvates}

\section{Andrew D. Bond and Changquan C. Sun}

\section{Computing details}

For all structures, data collection: APEX3 (Bruker, 2018); cell refinement: SAINT (Bruker, 2018); data reduction: SAINT (Bruker, 2018); program(s) used to solve structure: SHELXT (Sheldrick, 2015a); program(s) used to refine structure: SHELXL2018 (Sheldrick, 2015b); molecular graphics: Mercury (Macrae et al., 2020); software used to prepare material for publication: SHELXL2018 (Sheldrick, 2015b).

4-[5-(4-Methylphenyl)-3-(trifluoromethyl)pyrazol-1-yl]benzenesulfonamide dimethylformamide monosolvate (1)

Crystal data

$\mathrm{C}_{17} \mathrm{H}_{14} \mathrm{~F}_{3} \mathrm{~N}_{3} \mathrm{O}_{2} \mathrm{~S} \cdot \mathrm{C}_{3} \mathrm{H}_{7} \mathrm{NO}$

$M_{r}=454.47$

Monoclinic, $P 2{ }_{1} / c$

$a=11.8973(4) \AA$

$b=8.8360(3) \AA$

$c=21.8286(7) \AA$

$\beta=103.4537(13)^{\circ}$

$V=2231.75(13) \AA^{3}$

$Z=4$

Data collection

Bruker D8-QUEST PHOTON-100 diffractometer

Radiation source: Incoatec $\mathrm{I} \mu \mathrm{S} \mathrm{Cu}$ microsource $\omega$ and $\varphi$-scans Absorption correction: multi-scan

(SADABS; Bruker, 2016)

$T_{\min }=0.440, T_{\max }=0.753$

16627 measured reflections

Refinement

Refinement on $F^{2}$

Least-squares matrix: full

$R\left[F^{2}>2 \sigma\left(F^{2}\right)\right]=0.056$

$w R\left(F^{2}\right)=0.149$

$S=1.08$

3940 reflections

346 parameters

35 restraints

Primary atom site location: dual
$F(000)=944$

$D_{\mathrm{x}}=1.353 \mathrm{Mg} \mathrm{m}^{-3}$

$\mathrm{Cu} K \alpha$ radiation, $\lambda=1.54178 \AA$

Cell parameters from 9864 reflections

$\theta=3.8-66.7^{\circ}$

$\mu=1.77 \mathrm{~mm}^{-1}$

$T=298 \mathrm{~K}$

Block, colourless

$0.16 \times 0.16 \times 0.14 \mathrm{~mm}$

3940 independent reflections

3362 reflections with $I>2 \sigma(I)$

$R_{\text {int }}=0.053$

$\theta_{\max }=66.9^{\circ}, \theta_{\min }=3.8^{\circ}$

$h=-14 \rightarrow 13$

$k=-6 \rightarrow 10$

$l=-26 \rightarrow 25$

Secondary atom site location: difference Fourier map

Hydrogen site location: mixed

$\mathrm{H}$ atoms treated by a mixture of independent and constrained refinement

$w=1 /\left[\sigma^{2}\left(F_{\mathrm{o}}{ }^{2}\right)+(0.0529 P)^{2}+1.4975 P\right]$ where $P=\left(F_{\mathrm{o}}^{2}+2 F_{\mathrm{c}}^{2}\right) / 3$

$(\Delta / \sigma)_{\max }=0.007$

$\Delta \rho_{\max }=0.25 \mathrm{e}^{-3}$

$\Delta \rho_{\min }=-0.37$ e $\AA^{-3}$ 


\section{Special details}

Geometry. All esds (except the esd in the dihedral angle between two 1.s. planes) are estimated using the full covariance matrix. The cell esds are taken into account individually in the estimation of esds in distances, angles and torsion angles; correlations between esds in cell parameters are only used when they are defined by crystal symmetry. An approximate (isotropic) treatment of cell esds is used for estimating esds involving l.s. planes.

Fractional atomic coordinates and isotropic or equivalent isotropic displacement parameters $\left(\AA^{2}\right)$

\begin{tabular}{|c|c|c|c|c|c|}
\hline & $x$ & $y$ & $z$ & $U_{\text {iso }} * / U_{\text {eq }}$ & Occ. $(<1)$ \\
\hline S1 & $0.43324(5)$ & $0.72236(9)$ & $0.57626(3)$ & $0.0657(2)$ & \\
\hline $\mathrm{O} 1$ & $0.42145(18)$ & $0.6803(3)$ & $0.63714(10)$ & $0.0855(7)$ & \\
\hline $\mathrm{O} 2$ & $0.37685(17)$ & $0.8553(3)$ & $0.54695(13)$ & $0.0960(8)$ & \\
\hline N1 & $0.99334(18)$ & $0.8579(3)$ & $0.65536(10)$ & $0.0662(6)$ & \\
\hline $\mathrm{N} 2$ & $0.93631(16)$ & $0.8092(3)$ & $0.59763(10)$ & $0.0549(5)$ & \\
\hline N3 & $0.3870(2)$ & $0.5829(4)$ & $0.53040(14)$ & $0.0787(7)$ & \\
\hline H1N & $0.388(3)$ & $0.602(4)$ & $0.4916(7)$ & $0.105(13)^{*}$ & \\
\hline $\mathrm{H} 2 \mathrm{~N}$ & $0.420(3)$ & $0.501(3)$ & $0.5478(13)$ & $0.096(13)^{*}$ & \\
\hline $\mathrm{C} 1$ & $1.1007(2)$ & $0.8764(4)$ & $0.64939(14)$ & $0.0674(8)$ & \\
\hline $\mathrm{C} 2$ & $1.1140(2)$ & $0.8403(3)$ & $0.59008(14)$ & $0.0647(7)$ & \\
\hline $\mathrm{H} 2 \mathrm{~A}$ & 1.181655 & 0.845345 & 0.575755 & $0.078^{*}$ & \\
\hline $\mathrm{C} 3$ & $1.00633(19)$ & $0.7950(3)$ & $0.55629(12)$ & $0.0525(6)$ & \\
\hline $\mathrm{C} 4$ & $1.1897(3)$ & $0.9281(5)$ & $0.70607(18)$ & $0.0929(11)$ & \\
\hline $\mathrm{F} 1$ & $1.2098(12)$ & $1.0702(8)$ & $0.7055(8)$ & $0.214(10)$ & $0.430(12)$ \\
\hline $\mathrm{F} 2$ & $1.1623(7)$ & $0.9023(18)$ & $0.7598(3)$ & $0.161(8)$ & $0.430(12)$ \\
\hline F3 & $1.2858(6)$ & $0.8657(12)$ & $0.7111(4)$ & $0.103(4)$ & $0.430(12)$ \\
\hline F1A & $1.1645(9)$ & $1.0570(9)$ & $0.7240(3)$ & $0.133(4)$ & $0.570(12)$ \\
\hline F2A & $1.2070(13)$ & $0.8394(12)$ & $0.7517(5)$ & $0.260(9)$ & $0.570(12)$ \\
\hline F3A & $1.2861(7)$ & $0.952(2)$ & $0.6907(6)$ & $0.255(8)$ & $0.570(12)$ \\
\hline $\mathrm{C} 5$ & $0.97223(19)$ & $0.7359(3)$ & $0.49193(12)$ & $0.0525(6)$ & \\
\hline C6 & $0.8879(2)$ & $0.6253(3)$ & $0.47364(12)$ & $0.0552(6)$ & \\
\hline H6A & 0.848346 & 0.588556 & 0.502597 & $0.066^{*}$ & \\
\hline $\mathrm{C} 7$ & $0.8625(2)$ & $0.5699(3)$ & $0.41321(13)$ & $0.0622(7)$ & \\
\hline H7A & 0.804405 & 0.497839 & 0.401835 & $0.075^{*}$ & \\
\hline $\mathrm{C} 8$ & $0.9204(3)$ & $0.6177(4)$ & $0.36884(13)$ & $0.0690(7)$ & \\
\hline C9 & $1.0055(3)$ & $0.7265(4)$ & $0.38758(16)$ & $0.0824(9)$ & \\
\hline H9A & 1.046738 & 0.760708 & 0.358948 & $0.099^{*}$ & \\
\hline $\mathrm{C} 10$ & $1.0302(3)$ & $0.7845(4)$ & $0.44753(15)$ & $0.0719(8)$ & \\
\hline $\mathrm{H} 10 \mathrm{~A}$ & 1.087112 & 0.858253 & 0.458519 & $0.086^{*}$ & \\
\hline $\mathrm{C} 11$ & $0.8925(4)$ & $0.5548(5)$ & $0.30287(16)$ & $0.1031(12)$ & \\
\hline H11A & 0.953139 & 0.580762 & 0.282434 & $0.155^{*}$ & \\
\hline H11B & 0.820812 & 0.596789 & 0.279639 & $0.155^{*}$ & \\
\hline $\mathrm{H} 11 \mathrm{C}$ & 0.885819 & 0.446641 & 0.304506 & $0.155^{*}$ & \\
\hline $\mathrm{C} 12$ & 0.81447 (19) & $0.7846(3)$ & $0.58947(11)$ & $0.0505(6)$ & \\
\hline $\mathrm{C} 13$ & $0.7788(2)$ & $0.6962(4)$ & $0.63299(13)$ & $0.0666(8)$ & \\
\hline H13A & 0.832849 & 0.649861 & 0.665177 & $0.080^{*}$ & \\
\hline $\mathrm{C} 14$ & $0.6617(2)$ & $0.6764(4)$ & $0.62874(13)$ & $0.0650(7)$ & \\
\hline H14A & 0.636730 & 0.616960 & 0.658131 & $0.078^{*}$ & \\
\hline $\mathrm{C} 15$ & 0.58275 (19) & $0.7452(3)$ & $0.58074(11)$ & $0.0517(6)$ & \\
\hline
\end{tabular}




$\begin{array}{llllll}\text { C16 } & 0.61940(19) & 0.8334(3) & 0.53691(12) & 0.0528(6) & \\ \text { H16A } & 0.565515 & 0.879102 & 0.504437 & 0.063^{*} & \\ \text { C17 } & 0.73656(19) & 0.8539(3) & 0.54135(11) & 0.0522(6) & \\ \text { H17A } & 0.761968 & 0.913581 & 0.512178 & 0.063^{*} & \\ \text { O1S } & 0.4484(3) & 0.6595(3) & 0.41413(12) & 0.1112(9) & 0.779(7) \\ \text { N1S } & 0.4652(5) & 0.8198(6) & 0.3374(3) & 0.0814(14) & 0.779(7) \\ \text { C1S } & 0.4091(4) & 0.7692(6) & 0.3785(2) & 0.0842(15) & 0.779(7) \\ \text { H1S } & 0.340031 & 0.814639 & 0.381342 & 0.101^{*} & 0.779(7) \\ \text { C2S } & 0.5679(8) & 0.7485(9) & 0.3315(5) & 0.182(4) & 0.779(7) \\ \text { H2S } & 0.597691 & 0.798903 & 0.299680 & 0.272^{*} & 0.779(7) \\ \text { H3S } & 0.623841 & 0.753451 & 0.371019 & 0.272^{*} & 0.779(7) \\ \text { H4S } & 0.552215 & 0.644533 & 0.319836 & 0.272^{*} & 0.779(7) \\ \text { C3S } & 0.4288(5) & 0.9452(6) & 0.2954(2) & 0.1336(18) & 0.779(7) \\ \text { H5S } & 0.483661 & 0.960581 & 0.269917 & 0.200^{*} & 0.779(7) \\ \text { H6S } & 0.354239 & 0.923789 & 0.268657 & 0.200^{*} & 0.779(7) \\ \text { H7S } & 0.424206 & 1.034917 & 0.319466 & 0.200^{*} & 0.779(7) \\ \text { O1SA } & 0.4484(3) & 0.6595(3) & 0.41413(12) & 0.1112(9) & 0.221(7) \\ \text { N1SA } & 0.4178(12) & 0.829(2) & 0.3395(9) & 0.071(5) & 0.221(7) \\ \text { C1SA } & 0.4898(12) & 0.7281(18) & 0.3715(7) & 0.104(6) & 0.221(7) \\ \text { H8S } & 0.562389 & 0.707947 & 0.364335 & 0.124^{*} & 0.221(7) \\ \text { C2SA } & 0.3100(13) & 0.847(2) & 0.3560(9) & 0.127(9) & 0.221(7) \\ \text { H9S } & 0.265236 & 0.922855 & 0.329773 & 0.191^{*} & 0.221(7) \\ \text { H10S } & 0.268906 & 0.752317 & 0.349949 & 0.191^{*} & 0.221(7) \\ \text { H11S } & 0.322819 & 0.876604 & 0.399329 & 0.191^{*} & 0.221(7) \\ \text { C3SA } & 0.4288(5) & 0.9452(6) & 0.2954(2) & 0.1336(18) & 0.221(7) \\ \text { H12S } & 0.356405 & 0.997211 & 0.281819 & 0.200^{*} & 0.221(7) \\ \text { H13S } & 0.487444 & 1.015830 & 0.315055 & 0.200^{*} & 0.221(7) \\ \text { H14S } & 0.449868 & 0.900206 & 0.259591 & 0.200^{*} & 0.221(7)\end{array}$

Atomic displacement parameters $\left(\AA^{2}\right)$

\begin{tabular}{lllllll}
\hline & $U^{11}$ & $U^{22}$ & $U^{33}$ & $U^{12}$ & $U^{13}$ & $U^{23}$ \\
\hline S1 & $0.0357(3)$ & $0.0825(5)$ & $0.0850(5)$ & $0.0007(3)$ & $0.0262(3)$ & $0.0128(4)$ \\
O1 & $0.0602(12)$ & $0.1233(19)$ & $0.0855(14)$ & $-0.0024(12)$ & $0.0422(10)$ & $0.0075(13)$ \\
O2 & $0.0436(10)$ & $0.0949(17)$ & $0.158(2)$ & $0.0214(11)$ & $0.0402(12)$ & $0.0396(15)$ \\
N1 & $0.0417(11)$ & $0.0888(17)$ & $0.0647(13)$ & $-0.0017(11)$ & $0.0055(9)$ & $-0.0132(12)$ \\
N2 & $0.0319(9)$ & $0.0713(14)$ & $0.0600(12)$ & $-0.0019(9)$ & $0.0079(8)$ & $-0.0031(10)$ \\
N3 & $0.0513(14)$ & $0.099(2)$ & $0.0838(19)$ & $-0.0207(14)$ & $0.0110(12)$ & $0.0098(16)$ \\
C1 & $0.0359(12)$ & $0.0794(19)$ & $0.0827(18)$ & $-0.0013(12)$ & $0.0050(12)$ & $-0.0194(15)$ \\
C2 & $0.0318(11)$ & $0.0718(18)$ & $0.0902(19)$ & $-0.0041(12)$ & $0.0138(12)$ & $-0.0160(15)$ \\
C3 & $0.0332(11)$ & $0.0561(14)$ & $0.0680(15)$ & $-0.0006(10)$ & $0.0116(10)$ & $-0.0031(11)$ \\
C4 & $0.0530(19)$ & $0.114(3)$ & $0.104(3)$ & $-0.012(2)$ & $0.0043(17)$ & $-0.040(3)$ \\
F1 & $0.171(14)$ & $0.098(7)$ & $0.294(19)$ & $-0.048(7)$ & $-0.111(12)$ & $-0.013(8)$ \\
F2 & $0.077(4)$ & $0.35(2)$ & $0.064(5)$ & $-0.069(7)$ & $0.022(4)$ & $-0.068(9)$ \\
F3 & $0.051(4)$ & $0.148(7)$ & $0.090(4)$ & $0.035(4)$ & $-0.027(3)$ & $-0.033(4)$ \\
F1A & $0.154(7)$ & $0.140(7)$ & $0.079(4)$ & $0.028(5)$ & $-0.023(3)$ & $-0.059(4)$ \\
F2A & $0.266(15)$ & $0.195(8)$ & $0.209(12)$ & $-0.077(9)$ & $-0.169(11)$ & $0.082(8)$ \\
F3A & $0.070(5)$ & $0.44(2)$ & $0.268(11)$ & $-0.106(8)$ & $0.058(6)$ & $-0.239(12)$
\end{tabular}


supporting information

$\begin{array}{lllllll}\text { C5 } & 0.0348(11) & 0.0567(14) & 0.0670(15) & 0.0027(10) & 0.0142(10) & 0.0012(11) \\ \text { C6 } & 0.0398(12) & 0.0607(15) & 0.0665(15) & -0.0018(11) & 0.0152(11) & 0.0008(12) \\ \text { C7 } & 0.0461(13) & 0.0643(16) & 0.0738(17) & -0.0016(12) & 0.0088(12) & -0.0046(13) \\ \text { C8 } & 0.0632(16) & 0.0773(19) & 0.0665(16) & 0.0027(15) & 0.0148(13) & -0.0028(14) \\ \text { C9 } & 0.081(2) & 0.099(2) & 0.078(2) & -0.0124(18) & 0.0380(17) & 0.0037(18) \\ \text { C10 } & 0.0604(17) & 0.078(2) & 0.0825(19) & -0.0217(15) & 0.0281(14) & -0.0037(15) \\ \text { C11 } & 0.111(3) & 0.123(3) & 0.075(2) & -0.008(3) & 0.0220(19) & -0.014(2) \\ \text { C12 } & 0.0316(11) & 0.0640(15) & 0.0564(13) & -0.0008(10) & 0.0109(9) & 0.0004(11) \\ \text { C13 } & 0.0420(13) & 0.092(2) & 0.0632(15) & 0.0021(13) & 0.0067(11) & 0.0215(14) \\ \text { C14 } & 0.0475(14) & 0.087(2) & 0.0633(15) & -0.0051(13) & 0.0182(11) & 0.0211(14) \\ \text { C15 } & 0.0334(11) & 0.0649(15) & 0.0596(14) & -0.0006(10) & 0.0168(10) & 0.0046(11) \\ \text { C16 } & 0.0342(11) & 0.0638(15) & 0.0603(14) & 0.0030(11) & 0.0108(10) & 0.0097(12) \\ \text { C17 } & 0.0365(11) & 0.0623(15) & 0.0594(14) & -0.0023(11) & 0.0142(10) & 0.0096(11) \\ \text { O1S } & 0.138(2) & 0.111(2) & 0.0815(16) & -0.0067(18) & 0.0190(15) & 0.0189(15) \\ \text { N1S } & 0.090(4) & 0.079(3) & 0.074(3) & -0.003(3) & 0.017(3) & 0.001(2) \\ \text { C1S } & 0.072(3) & 0.102(4) & 0.073(3) & 0.008(2) & 0.007(2) & -0.015(3) \\ \text { C2S } & 0.188(8) & 0.154(7) & 0.255(10) & 0.052(6) & 0.156(8) & 0.077(7) \\ \text { C3S } & 0.167(5) & 0.112(3) & 0.104(3) & -0.014(3) & -0.006(3) & 0.025(3) \\ \text { O1SA } & 0.138(2) & 0.111(2) & 0.0815(16) & -0.0067(18) & 0.0190(15) & 0.0189(15) \\ \text { N1SA } & 0.071(9) & 0.080(8) & 0.060(7) & -0.013(7) & 0.012(6) & 0.003(6) \\ \text { C1SA } & 0.101(10) & 0.116(10) & 0.096(9) & -0.004(8) & 0.029(8) & 0.001(8) \\ \text { C2SA } & 0.097(14) & 0.16(2) & 0.117(15) & 0.056(14) & 0.005(11) & -0.033(13) \\ \text { C3SA } & 0.167(5) & 0.112(3) & 0.104(3) & -0.014(3) & -0.006(3) & 0.025(3)\end{array}$

Geometric parameters $\left(\AA,{ }^{o}\right)$

\begin{tabular}{llll}
\hline $\mathrm{S} 1-\mathrm{O} 1$ & $1.418(2)$ & $\mathrm{C} 11-\mathrm{H} 11 \mathrm{C}$ & 0.9600 \\
$\mathrm{~S} 1-\mathrm{O} 2$ & $1.428(2)$ & $\mathrm{C} 12-\mathrm{C} 17$ & $1.372(3)$ \\
$\mathrm{S} 1-\mathrm{N} 3$ & $1.602(3)$ & $\mathrm{C} 12-\mathrm{C} 13$ & $1.372(4)$ \\
$\mathrm{S} 1-\mathrm{C} 15$ & $1.770(2)$ & $\mathrm{C} 13-\mathrm{C} 14$ & $1.385(4)$ \\
$\mathrm{N} 1-\mathrm{C} 1$ & $1.324(3)$ & $\mathrm{C} 13-\mathrm{H} 13 \mathrm{~A}$ & 0.9300 \\
$\mathrm{~N} 1-\mathrm{N} 2$ & $1.355(3)$ & $\mathrm{C} 14-\mathrm{C} 15$ & $1.376(4)$ \\
$\mathrm{N} 2-\mathrm{C} 3$ & $1.369(3)$ & $\mathrm{C} 14-\mathrm{H} 14 \mathrm{~A}$ & 0.9300 \\
$\mathrm{~N} 2-\mathrm{C} 12$ & $1.435(3)$ & $\mathrm{C} 15-\mathrm{C} 16$ & $1.381(3)$ \\
$\mathrm{N} 3-\mathrm{H} 1 \mathrm{~N}$ & $0.866(10)$ & $\mathrm{C} 16-\mathrm{C} 17$ & $1.386(3)$ \\
$\mathrm{N} 3-\mathrm{H} 2 \mathrm{~N}$ & $0.869(10)$ & $\mathrm{C} 16-\mathrm{H} 16 \mathrm{~A}$ & 0.9300 \\
$\mathrm{C} 1-\mathrm{C} 2$ & $1.378(4)$ & $\mathrm{C} 17-\mathrm{H} 17 \mathrm{~A}$ & 0.9300 \\
$\mathrm{C} 1-\mathrm{C} 4$ & $1.501(4)$ & $\mathrm{O} 1 \mathrm{~S}-\mathrm{C} 1 \mathrm{~S}$ & $1.262(5)$ \\
$\mathrm{C} 2-\mathrm{C} 3$ & $1.380(3)$ & $\mathrm{N} 1 \mathrm{~S}-\mathrm{C} 1 \mathrm{~S}$ & $1.314(6)$ \\
$\mathrm{C} 2-\mathrm{H} 2 \mathrm{~A}$ & 0.9300 & $\mathrm{~N} 1 \mathrm{~S}-\mathrm{C} 2 \mathrm{~S}$ & $1.407(9)$ \\
$\mathrm{C} 3-\mathrm{C} 5$ & $1.465(4)$ & $\mathrm{N} 1 \mathrm{~S}-\mathrm{C} 3 \mathrm{~S}$ & $1.440(6)$ \\
$\mathrm{C} 4-\mathrm{F} 2 \mathrm{~A}$ & $1.246(7)$ & $\mathrm{C} 1 \mathrm{~S}-\mathrm{H} 1 \mathrm{~S}$ & 0.9300 \\
$\mathrm{C} 4-\mathrm{F} 3$ & $1.250(6)$ & $\mathrm{C} 2 \mathrm{~S}-\mathrm{H} 2 \mathrm{~S}$ & 0.9600 \\
$\mathrm{C} 4-\mathrm{F} 1 \mathrm{~A}$ & $1.263(6)$ & $\mathrm{C} 2 \mathrm{~S}-\mathrm{H} 3 \mathrm{~S}$ & 0.9600 \\
$\mathrm{C} 4-\mathrm{F} 1$ & $1.278(8)$ & $\mathrm{C} 2 \mathrm{~S}-\mathrm{H} 4 \mathrm{~S}$ & 0.9600 \\
$\mathrm{C} 4-\mathrm{F} 3 \mathrm{~A}$ & $1.285(7)$ & $\mathrm{C} 3 \mathrm{~S}-\mathrm{H} 5 \mathrm{~S}$ & 0.9600 \\
$\mathrm{C} 4-\mathrm{F} 2$ & $1.309(7)$ & $\mathrm{C} 3 \mathrm{~S}-\mathrm{H} 6 \mathrm{~S}$ & 0.9600 \\
$\mathrm{C} 5-\mathrm{C} 10$ & $1.382(4)$ & $\mathrm{C} 3 \mathrm{~S}-\mathrm{H} 7 \mathrm{~S}$ & 0.9600
\end{tabular}




\begin{tabular}{|c|c|c|c|}
\hline $\mathrm{C} 5-\mathrm{C} 6$ & $1.391(3)$ & $\mathrm{O} 1 \mathrm{SA}-\mathrm{C} 1 \mathrm{SA}$ & $1.299(10)$ \\
\hline $\mathrm{C} 6-\mathrm{C} 7$ & $1.373(4)$ & $\mathrm{N} 1 \mathrm{SA}-\mathrm{C} 1 \mathrm{SA}$ & $1.321(10)$ \\
\hline C6-H6A & 0.9300 & $\mathrm{~N} 1 \mathrm{SA}-\mathrm{C} 2 \mathrm{SA}$ & $1.419(12)$ \\
\hline $\mathrm{C} 7-\mathrm{C} 8$ & $1.379(4)$ & $\mathrm{N} 1 \mathrm{SA}-\mathrm{C} 3 \mathrm{SA}$ & $1.432(9)$ \\
\hline C7-H7A & 0.9300 & $\mathrm{C} 1 \mathrm{SA}-\mathrm{H} 8 \mathrm{~S}$ & 0.9300 \\
\hline $\mathrm{C} 8-\mathrm{C} 9$ & $1.387(4)$ & $\mathrm{C} 2 \mathrm{SA}-\mathrm{H} 9 \mathrm{~S}$ & 0.9600 \\
\hline $\mathrm{C} 8-\mathrm{C} 11$ & $1.507(4)$ & $\mathrm{C} 2 \mathrm{SA}-\mathrm{H} 10 \mathrm{~S}$ & 0.9600 \\
\hline $\mathrm{C} 9-\mathrm{C} 10$ & $1.372(4)$ & $\mathrm{C} 2 \mathrm{SA}-\mathrm{H} 11 \mathrm{~S}$ & 0.9600 \\
\hline C9-H9A & 0.9300 & $\mathrm{C} 3 \mathrm{SA}-\mathrm{H} 12 \mathrm{~S}$ & 0.9600 \\
\hline $\mathrm{C} 10-\mathrm{H} 10 \mathrm{~A}$ & 0.9300 & $\mathrm{C} 3 \mathrm{SA}-\mathrm{H} 13 \mathrm{~S}$ & 0.9600 \\
\hline C11-H11A & 0.9600 & $\mathrm{C} 3 \mathrm{SA}-\mathrm{H} 14 \mathrm{~S}$ & 0.9600 \\
\hline $\mathrm{C} 11-\mathrm{H} 11 \mathrm{~B}$ & 0.9600 & & \\
\hline $\mathrm{O} 1-\mathrm{S} 1-\mathrm{O} 2$ & $119.78(15)$ & $\mathrm{H} 11 \mathrm{~A}-\mathrm{C} 11-\mathrm{H} 11 \mathrm{C}$ & 109.5 \\
\hline $\mathrm{O} 1-\mathrm{S} 1-\mathrm{N} 3$ & $106.84(15)$ & $\mathrm{H} 11 \mathrm{~B}-\mathrm{C} 11-\mathrm{H} 11 \mathrm{C}$ & 109.5 \\
\hline $\mathrm{O} 2-\mathrm{S} 1-\mathrm{N} 3$ & $107.20(17)$ & $\mathrm{C} 17-\mathrm{C} 12-\mathrm{C} 13$ & $121.4(2)$ \\
\hline $\mathrm{O} 1-\mathrm{S} 1-\mathrm{C} 15$ & $107.17(12)$ & $\mathrm{C} 17-\mathrm{C} 12-\mathrm{N} 2$ & $120.7(2)$ \\
\hline $\mathrm{O} 2-\mathrm{S} 1-\mathrm{C} 15$ & $106.99(12)$ & $\mathrm{C} 13-\mathrm{C} 12-\mathrm{N} 2$ & $117.8(2)$ \\
\hline $\mathrm{N} 3-\mathrm{S} 1-\mathrm{C} 15$ & $108.46(13)$ & $\mathrm{C} 12-\mathrm{C} 13-\mathrm{C} 14$ & $119.6(2)$ \\
\hline $\mathrm{C} 1-\mathrm{N} 1-\mathrm{N} 2$ & $103.5(2)$ & $\mathrm{C} 12-\mathrm{C} 13-\mathrm{H} 13 \mathrm{~A}$ & 120.2 \\
\hline $\mathrm{N} 1-\mathrm{N} 2-\mathrm{C} 3$ & $113.10(19)$ & $\mathrm{C} 14-\mathrm{C} 13-\mathrm{H} 13 \mathrm{~A}$ & 120.2 \\
\hline $\mathrm{N} 1-\mathrm{N} 2-\mathrm{C} 12$ & $115.86(19)$ & $\mathrm{C} 15-\mathrm{C} 14-\mathrm{C} 13$ & $119.6(2)$ \\
\hline $\mathrm{C} 3-\mathrm{N} 2-\mathrm{C} 12$ & $131.0(2)$ & $\mathrm{C} 15-\mathrm{C} 14-\mathrm{H} 14 \mathrm{~A}$ & 120.2 \\
\hline $\mathrm{S} 1-\mathrm{N} 3-\mathrm{H} 1 \mathrm{~N}$ & $112(2)$ & $\mathrm{C} 13-\mathrm{C} 14-\mathrm{H} 14 \mathrm{~A}$ & 120.2 \\
\hline $\mathrm{S} 1-\mathrm{N} 3-\mathrm{H} 2 \mathrm{~N}$ & $108(2)$ & $\mathrm{C} 14-\mathrm{C} 15-\mathrm{C} 16$ & $120.5(2)$ \\
\hline $\mathrm{H} 1 \mathrm{~N}-\mathrm{N} 3-\mathrm{H} 2 \mathrm{~N}$ & $118.7(18)$ & $\mathrm{C} 14-\mathrm{C} 15-\mathrm{S} 1$ & $119.44(18)$ \\
\hline $\mathrm{N} 1-\mathrm{C} 1-\mathrm{C} 2$ & $112.8(2)$ & $\mathrm{C} 16-\mathrm{C} 15-\mathrm{S} 1$ & $120.08(18)$ \\
\hline $\mathrm{N} 1-\mathrm{C} 1-\mathrm{C} 4$ & $117.9(3)$ & $\mathrm{C} 15-\mathrm{C} 16-\mathrm{C} 17$ & $120.0(2)$ \\
\hline $\mathrm{C} 2-\mathrm{C} 1-\mathrm{C} 4$ & $129.2(3)$ & $\mathrm{C} 15-\mathrm{C} 16-\mathrm{H} 16 \mathrm{~A}$ & 120.0 \\
\hline $\mathrm{C} 1-\mathrm{C} 2-\mathrm{C} 3$ & $105.9(2)$ & $\mathrm{C} 17-\mathrm{C} 16-\mathrm{H} 16 \mathrm{~A}$ & 120.0 \\
\hline $\mathrm{C} 1-\mathrm{C} 2-\mathrm{H} 2 \mathrm{~A}$ & 127.0 & $\mathrm{C} 12-\mathrm{C} 17-\mathrm{C} 16$ & $119.0(2)$ \\
\hline $\mathrm{C} 3-\mathrm{C} 2-\mathrm{H} 2 \mathrm{~A}$ & 127.0 & $\mathrm{C} 12-\mathrm{C} 17-\mathrm{H} 17 \mathrm{~A}$ & 120.5 \\
\hline $\mathrm{N} 2-\mathrm{C} 3-\mathrm{C} 2$ & $104.6(2)$ & $\mathrm{C} 16-\mathrm{C} 17-\mathrm{H} 17 \mathrm{~A}$ & 120.5 \\
\hline $\mathrm{N} 2-\mathrm{C} 3-\mathrm{C} 5$ & $126.1(2)$ & $\mathrm{C} 1 \mathrm{~S}-\mathrm{N} 1 \mathrm{~S}-\mathrm{C} 2 \mathrm{~S}$ & $120.0(5)$ \\
\hline $\mathrm{C} 2-\mathrm{C} 3-\mathrm{C} 5$ & $129.2(2)$ & $\mathrm{C} 1 \mathrm{~S}-\mathrm{N} 1 \mathrm{~S}-\mathrm{C} 3 \mathrm{~S}$ & $124.9(5)$ \\
\hline $\mathrm{F} 2 \mathrm{~A}-\mathrm{C} 4-\mathrm{F} 1 \mathrm{~A}$ & $109.0(7)$ & $\mathrm{C} 2 \mathrm{~S}-\mathrm{N} 1 \mathrm{~S}-\mathrm{C} 3 \mathrm{~S}$ & $115.2(5)$ \\
\hline $\mathrm{F} 3-\mathrm{C} 4-\mathrm{F} 1$ & $105.4(7)$ & $\mathrm{O} 1 \mathrm{~S}-\mathrm{C} 1 \mathrm{~S}-\mathrm{N} 1 \mathrm{~S}$ & $120.5(5)$ \\
\hline $\mathrm{F} 2 \mathrm{~A}-\mathrm{C} 4-\mathrm{F} 3 \mathrm{~A}$ & $108.7(6)$ & $\mathrm{O} 1 \mathrm{~S}-\mathrm{C} 1 \mathrm{~S}-\mathrm{H} 1 \mathrm{~S}$ & 119.7 \\
\hline $\mathrm{F} 1 \mathrm{~A}-\mathrm{C} 4-\mathrm{F} 3 \mathrm{~A}$ & $102.9(6)$ & $\mathrm{N} 1 \mathrm{~S}-\mathrm{C} 1 \mathrm{~S}-\mathrm{H} 1 \mathrm{~S}$ & 119.7 \\
\hline $\mathrm{F} 3-\mathrm{C} 4-\mathrm{F} 2$ & $104.9(6)$ & $\mathrm{N} 1 \mathrm{~S}-\mathrm{C} 2 \mathrm{~S}-\mathrm{H} 2 \mathrm{~S}$ & 109.5 \\
\hline $\mathrm{F} 1-\mathrm{C} 4-\mathrm{F} 2$ & $105.4(7)$ & $\mathrm{N} 1 \mathrm{~S}-\mathrm{C} 2 \mathrm{~S}-\mathrm{H} 3 \mathrm{~S}$ & 109.5 \\
\hline $\mathrm{F} 2 \mathrm{~A}-\mathrm{C} 4-\mathrm{C} 1$ & $114.9(5)$ & $\mathrm{H} 2 \mathrm{~S}-\mathrm{C} 2 \mathrm{~S}-\mathrm{H} 3 \mathrm{~S}$ & 109.5 \\
\hline $\mathrm{F} 3-\mathrm{C} 4-\mathrm{C} 1$ & $113.2(4)$ & $\mathrm{N} 1 \mathrm{~S}-\mathrm{C} 2 \mathrm{~S}-\mathrm{H} 4 \mathrm{~S}$ & 109.5 \\
\hline $\mathrm{F} 1 \mathrm{~A}-\mathrm{C} 4-\mathrm{C} 1$ & $111.0(4)$ & $\mathrm{H} 2 \mathrm{~S}-\mathrm{C} 2 \mathrm{~S}-\mathrm{H} 4 \mathrm{~S}$ & 109.5 \\
\hline $\mathrm{F} 1-\mathrm{C} 4-\mathrm{C} 1$ & $112.9(7)$ & $\mathrm{H} 3 \mathrm{~S}-\mathrm{C} 2 \mathrm{~S}-\mathrm{H} 4 \mathrm{~S}$ & 109.5 \\
\hline $\mathrm{F} 3 \mathrm{~A}-\mathrm{C} 4-\mathrm{C} 1$ & $109.7(5)$ & $\mathrm{N} 1 \mathrm{~S}-\mathrm{C} 3 \mathrm{~S}-\mathrm{H} 5 \mathrm{~S}$ & 109.5 \\
\hline $\mathrm{F} 2-\mathrm{C} 4-\mathrm{C} 1$ & $114.2(4)$ & $\mathrm{N} 1 \mathrm{~S}-\mathrm{C} 3 \mathrm{~S}-\mathrm{H} 6 \mathrm{~S}$ & 109.5 \\
\hline $\mathrm{C} 10-\mathrm{C} 5-\mathrm{C} 6$ & $117.5(2)$ & $\mathrm{H} 5 \mathrm{~S}-\mathrm{C} 3 \mathrm{~S}-\mathrm{H} 6 \mathrm{~S}$ & 109.5 \\
\hline
\end{tabular}




\begin{tabular}{|c|c|c|c|}
\hline $\mathrm{C} 10-\mathrm{C} 5-\mathrm{C} 3$ & $119.3(2)$ & $\mathrm{N} 1 \mathrm{~S}-\mathrm{C} 3 \mathrm{~S}-\mathrm{H} 7 \mathrm{~S}$ & 109.5 \\
\hline $\mathrm{C} 6-\mathrm{C} 5-\mathrm{C} 3$ & $123.1(2)$ & $\mathrm{H} 5 \mathrm{~S}-\mathrm{C} 3 \mathrm{~S}-\mathrm{H} 7 \mathrm{~S}$ & 109.5 \\
\hline $\mathrm{C} 7-\mathrm{C} 6-\mathrm{C} 5$ & $120.6(2)$ & $\mathrm{H} 6 \mathrm{~S}-\mathrm{C} 3 \mathrm{~S}-\mathrm{H} 7 \mathrm{~S}$ & 109.5 \\
\hline $\mathrm{C} 7-\mathrm{C} 6-\mathrm{H} 6 \mathrm{~A}$ & 119.7 & $\mathrm{C} 1 \mathrm{SA}-\mathrm{N} 1 \mathrm{SA}-\mathrm{C} 2 \mathrm{SA}$ & $116.9(10)$ \\
\hline $\mathrm{C} 5-\mathrm{C} 6-\mathrm{H} 6 \mathrm{~A}$ & 119.7 & $\mathrm{C} 1 \mathrm{SA}-\mathrm{N} 1 \mathrm{SA}-\mathrm{C} 3 \mathrm{SA}$ & $133.7(10)$ \\
\hline $\mathrm{C} 6-\mathrm{C} 7-\mathrm{C} 8$ & $122.1(3)$ & $\mathrm{C} 2 \mathrm{SA}-\mathrm{N} 1 \mathrm{SA}-\mathrm{C} 3 \mathrm{SA}$ & $108.8(10)$ \\
\hline $\mathrm{C} 6-\mathrm{C} 7-\mathrm{H} 7 \mathrm{~A}$ & 119.0 & $\mathrm{O} 1 \mathrm{SA}-\mathrm{C} 1 \mathrm{SA}-\mathrm{N} 1 \mathrm{SA}$ & $112.8(9)$ \\
\hline $\mathrm{C} 8-\mathrm{C} 7-\mathrm{H} 7 \mathrm{~A}$ & 119.0 & $\mathrm{O} 1 \mathrm{SA}-\mathrm{C} 1 \mathrm{SA}-\mathrm{H} 8 \mathrm{~S}$ & 123.6 \\
\hline $\mathrm{C} 7-\mathrm{C} 8-\mathrm{C} 9$ & $117.1(3)$ & $\mathrm{N} 1 \mathrm{SA}-\mathrm{C} 1 \mathrm{SA}-\mathrm{H} 8 \mathrm{~S}$ & 123.6 \\
\hline $\mathrm{C} 7-\mathrm{C} 8-\mathrm{C} 11$ & $121.5(3)$ & $\mathrm{N} 1 \mathrm{SA}-\mathrm{C} 2 \mathrm{SA}-\mathrm{H} 9 \mathrm{~S}$ & 109.5 \\
\hline $\mathrm{C} 9-\mathrm{C} 8-\mathrm{C} 11$ & $121.4(3)$ & $\mathrm{N} 1 \mathrm{SA}-\mathrm{C} 2 \mathrm{SA}-\mathrm{H} 10 \mathrm{~S}$ & 109.5 \\
\hline $\mathrm{C} 10-\mathrm{C} 9-\mathrm{C} 8$ & $121.3(3)$ & $\mathrm{H} 9 \mathrm{~S}-\mathrm{C} 2 \mathrm{SA}-\mathrm{H} 10 \mathrm{~S}$ & 109.5 \\
\hline $\mathrm{C} 10-\mathrm{C} 9-\mathrm{H} 9 \mathrm{~A}$ & 119.3 & $\mathrm{~N} 1 \mathrm{SA}-\mathrm{C} 2 \mathrm{SA}-\mathrm{H} 11 \mathrm{~S}$ & 109.5 \\
\hline $\mathrm{C} 8-\mathrm{C} 9-\mathrm{H} 9 \mathrm{~A}$ & 119.3 & $\mathrm{H} 9 \mathrm{~S}-\mathrm{C} 2 \mathrm{SA}-\mathrm{H} 11 \mathrm{~S}$ & 109.5 \\
\hline $\mathrm{C} 9-\mathrm{C} 10-\mathrm{C} 5$ & $121.4(3)$ & $\mathrm{H} 10 \mathrm{~S}-\mathrm{C} 2 \mathrm{SA}-\mathrm{H} 11 \mathrm{~S}$ & 109.5 \\
\hline $\mathrm{C} 9-\mathrm{C} 10-\mathrm{H} 10 \mathrm{~A}$ & 119.3 & $\mathrm{~N} 1 \mathrm{SA}-\mathrm{C} 3 \mathrm{SA}-\mathrm{H} 12 \mathrm{~S}$ & 109.5 \\
\hline $\mathrm{C} 5-\mathrm{C} 10-\mathrm{H} 10 \mathrm{~A}$ & 119.3 & $\mathrm{~N} 1 \mathrm{SA}-\mathrm{C} 3 \mathrm{SA}-\mathrm{H} 13 \mathrm{~S}$ & 109.5 \\
\hline $\mathrm{C} 8-\mathrm{C} 11-\mathrm{H} 11 \mathrm{~A}$ & 109.5 & $\mathrm{H} 12 \mathrm{~S}-\mathrm{C} 3 \mathrm{SA}-\mathrm{H} 13 \mathrm{~S}$ & 109.5 \\
\hline $\mathrm{C} 8-\mathrm{C} 11-\mathrm{H} 11 \mathrm{~B}$ & 109.5 & $\mathrm{~N} 1 \mathrm{SA}-\mathrm{C} 3 \mathrm{SA}-\mathrm{H} 14 \mathrm{~S}$ & 109.5 \\
\hline $\mathrm{H} 11 \mathrm{~A}-\mathrm{C} 11-\mathrm{H} 11 \mathrm{~B}$ & 109.5 & $\mathrm{H} 12 \mathrm{~S}-\mathrm{C} 3 \mathrm{SA}-\mathrm{H} 14 \mathrm{~S}$ & 109.5 \\
\hline $\mathrm{C} 8-\mathrm{C} 11-\mathrm{H} 11 \mathrm{C}$ & 109.5 & $\mathrm{H} 13 \mathrm{~S}-\mathrm{C} 3 \mathrm{SA}-\mathrm{H} 14 \mathrm{~S}$ & 109.5 \\
\hline $\mathrm{C} 1-\mathrm{N} 1-\mathrm{N} 2-\mathrm{C} 3$ & $0.9(3)$ & $\mathrm{C} 6-\mathrm{C} 7-\mathrm{C} 8-\mathrm{C} 9$ & $-0.7(4)$ \\
\hline $\mathrm{C} 1-\mathrm{N} 1-\mathrm{N} 2-\mathrm{C} 12$ & $-177.8(2)$ & $\mathrm{C} 6-\mathrm{C} 7-\mathrm{C} 8-\mathrm{C} 11$ & $179.4(3)$ \\
\hline $\mathrm{N} 2-\mathrm{N} 1-\mathrm{C} 1-\mathrm{C} 2$ & $-0.4(3)$ & $\mathrm{C} 7-\mathrm{C} 8-\mathrm{C} 9-\mathrm{C} 10$ & $-0.5(5)$ \\
\hline $\mathrm{N} 2-\mathrm{N} 1-\mathrm{C} 1-\mathrm{C} 4$ & $-178.9(3)$ & $\mathrm{C} 11-\mathrm{C} 8-\mathrm{C} 9-\mathrm{C} 10$ & $179.4(3)$ \\
\hline $\mathrm{N} 1-\mathrm{C} 1-\mathrm{C} 2-\mathrm{C} 3$ & $-0.2(4)$ & $\mathrm{C} 8-\mathrm{C} 9-\mathrm{C} 10-\mathrm{C} 5$ & $0.8(5)$ \\
\hline $\mathrm{C} 4-\mathrm{C} 1-\mathrm{C} 2-\mathrm{C} 3$ & $178.1(3)$ & $\mathrm{C} 6-\mathrm{C} 5-\mathrm{C} 10-\mathrm{C} 9$ & $0.1(4)$ \\
\hline $\mathrm{N} 1-\mathrm{N} 2-\mathrm{C} 3-\mathrm{C} 2$ & $-1.0(3)$ & $\mathrm{C} 3-\mathrm{C} 5-\mathrm{C} 10-\mathrm{C} 9$ & $176.5(3)$ \\
\hline $\mathrm{C} 12-\mathrm{N} 2-\mathrm{C} 3-\mathrm{C} 2$ & $177.4(3)$ & $\mathrm{N} 1-\mathrm{N} 2-\mathrm{C} 12-\mathrm{C} 17$ & $125.6(3)$ \\
\hline $\mathrm{N} 1-\mathrm{N} 2-\mathrm{C} 3-\mathrm{C} 5$ & $175.8(2)$ & $\mathrm{C} 3-\mathrm{N} 2-\mathrm{C} 12-\mathrm{C} 17$ & $-52.8(4)$ \\
\hline $\mathrm{C} 12-\mathrm{N} 2-\mathrm{C} 3-\mathrm{C} 5$ & $-5.8(4)$ & $\mathrm{N} 1-\mathrm{N} 2-\mathrm{C} 12-\mathrm{C} 13$ & $-51.3(3)$ \\
\hline $\mathrm{C} 1-\mathrm{C} 2-\mathrm{C} 3-\mathrm{N} 2$ & $0.7(3)$ & $\mathrm{C} 3-\mathrm{N} 2-\mathrm{C} 12-\mathrm{C} 13$ & $130.4(3)$ \\
\hline $\mathrm{C} 1-\mathrm{C} 2-\mathrm{C} 3-\mathrm{C} 5$ & $-176.0(3)$ & $\mathrm{C} 17-\mathrm{C} 12-\mathrm{C} 13-\mathrm{C} 14$ & $-0.3(4)$ \\
\hline $\mathrm{N} 1-\mathrm{C} 1-\mathrm{C} 4-\mathrm{F} 2 \mathrm{~A}$ & $62.5(11)$ & $\mathrm{N} 2-\mathrm{C} 12-\mathrm{C} 13-\mathrm{C} 14$ & $176.6(3)$ \\
\hline $\mathrm{C} 2-\mathrm{C} 1-\mathrm{C} 4-\mathrm{F} 2 \mathrm{~A}$ & $-115.7(11)$ & $\mathrm{C} 12-\mathrm{C} 13-\mathrm{C} 14-\mathrm{C} 15$ & $0.3(5)$ \\
\hline $\mathrm{N} 1-\mathrm{C} 1-\mathrm{C} 4-\mathrm{F} 3$ & $141.5(7)$ & $\mathrm{C} 13-\mathrm{C} 14-\mathrm{C} 15-\mathrm{C} 16$ & $0.1(4)$ \\
\hline $\mathrm{C} 2-\mathrm{C} 1-\mathrm{C} 4-\mathrm{F} 3$ & $-36.8(8)$ & $\mathrm{C} 13-\mathrm{C} 14-\mathrm{C} 15-\mathrm{S} 1$ & $-178.9(2)$ \\
\hline $\mathrm{N} 1-\mathrm{C} 1-\mathrm{C} 4-\mathrm{F} 1 \mathrm{~A}$ & $-61.7(8)$ & $\mathrm{O} 1-\mathrm{S} 1-\mathrm{C} 15-\mathrm{C} 14$ & $21.2(3)$ \\
\hline $\mathrm{C} 2-\mathrm{C} 1-\mathrm{C} 4-\mathrm{F} 1 \mathrm{~A}$ & $120.1(7)$ & $\mathrm{O} 2-\mathrm{S} 1-\mathrm{C} 15-\mathrm{C} 14$ & $150.9(2)$ \\
\hline $\mathrm{N} 1-\mathrm{C} 1-\mathrm{C} 4-\mathrm{F} 1$ & $-98.9(10)$ & $\mathrm{N} 3-\mathrm{S} 1-\mathrm{C} 15-\mathrm{C} 14$ & $-93.8(3)$ \\
\hline $\mathrm{C} 2-\mathrm{C} 1-\mathrm{C} 4-\mathrm{F} 1$ & $82.8(11)$ & $\mathrm{O} 1-\mathrm{S} 1-\mathrm{C} 15-\mathrm{C} 16$ & $-157.7(2)$ \\
\hline $\mathrm{N} 1-\mathrm{C} 1-\mathrm{C} 4-\mathrm{F} 3 \mathrm{~A}$ & $-174.7(10)$ & $\mathrm{O} 2-\mathrm{S} 1-\mathrm{C} 15-\mathrm{C} 16$ & $-28.1(3)$ \\
\hline $\mathrm{C} 2-\mathrm{C} 1-\mathrm{C} 4-\mathrm{F} 3 \mathrm{~A}$ & $7.0(11)$ & $\mathrm{N} 3-\mathrm{S} 1-\mathrm{C} 15-\mathrm{C} 16$ & $87.3(3)$ \\
\hline $\mathrm{N} 1-\mathrm{C} 1-\mathrm{C} 4-\mathrm{F} 2$ & $21.5(9)$ & $\mathrm{C} 14-\mathrm{C} 15-\mathrm{C} 16-\mathrm{C} 17$ & $-0.4(4)$ \\
\hline $\mathrm{C} 2-\mathrm{C} 1-\mathrm{C} 4-\mathrm{F} 2$ & $-156.8(8)$ & $\mathrm{S} 1-\mathrm{C} 15-\mathrm{C} 16-\mathrm{C} 17$ & $178.6(2)$ \\
\hline $\mathrm{N} 2-\mathrm{C} 3-\mathrm{C} 5-\mathrm{C} 10$ & $152.5(3)$ & $\mathrm{C} 13-\mathrm{C} 12-\mathrm{C} 17-\mathrm{C} 16$ & $0.0(4)$ \\
\hline $\mathrm{C} 2-\mathrm{C} 3-\mathrm{C} 5-\mathrm{C} 10$ & $-31.4(4)$ & $\mathrm{N} 2-\mathrm{C} 12-\mathrm{C} 17-\mathrm{C} 16$ & $-176.8(2)$ \\
\hline
\end{tabular}




$\begin{array}{llll}\mathrm{N} 2-\mathrm{C} 3-\mathrm{C} 5-\mathrm{C} 6 & -31.4(4) & \mathrm{C} 15-\mathrm{C} 16-\mathrm{C} 17-\mathrm{C} 12 & 0.4(4) \\ \mathrm{C} 2-\mathrm{C} 3-\mathrm{C} 5-\mathrm{C} 6 & 144.7(3) & \mathrm{C} 2 \mathrm{~S}-\mathrm{N} 1 \mathrm{~S}-\mathrm{C} 1 \mathrm{~S}-\mathrm{O} 1 \mathrm{~S} & 2.0(10) \\ \mathrm{C} 10-\mathrm{C} 5-\mathrm{C} 6-\mathrm{C} 7 & -1.3(4) & \mathrm{C} 3 \mathrm{~S}-\mathrm{N} 1 \mathrm{~S}-\mathrm{C} 1 \mathrm{~S}-\mathrm{O} 1 \mathrm{~S} & -179.0(5) \\ \mathrm{C} 3-\mathrm{C} 5-\mathrm{C} 6-\mathrm{C} 7 & -177.5(2) & \mathrm{C} 2 \mathrm{SA}-\mathrm{N} 1 \mathrm{SA}-\mathrm{C} 1 \mathrm{SA}-\mathrm{O} 1 \mathrm{SA} & 0(3) \\ \mathrm{C} 5-\mathrm{C} 6-\mathrm{C} 7-\mathrm{C} 8 & 1.6(4) & \mathrm{C} 3 \mathrm{SA}-\mathrm{N} 1 \mathrm{SA}-\mathrm{C} 1 \mathrm{SA}-\mathrm{O} 1 \mathrm{SA} & 170(2)\end{array}$

Hydrogen-bond geometry $\left(\AA,{ }^{\circ}\right)$

\begin{tabular}{lllll}
\hline$D-\mathrm{H} \cdots A$ & $D-\mathrm{H}$ & $\mathrm{H} \cdots A$ & $D \cdots A$ & $D-\mathrm{H} \cdots A$ \\
\hline $\mathrm{N} 3-\mathrm{H} 1 N \cdots \mathrm{O} 1 \mathrm{Sa}$ & $0.87(1)$ & $2.05(2)$ & $2.880(4)$ & $161(4)$ \\
$\mathrm{N} 3-\mathrm{H} 2 N \cdots \mathrm{O} 1 \mathrm{Sa}^{\mathrm{i}}$ & $0.87(1)$ & $2.13(2)$ & $2.964(4)$ & $160(3)$ \\
\hline
\end{tabular}

Symmetry code: (i) $-x+1,-y+1,-z+1$.

4-[5-(4-Methylphenyl)-3-(trifluoromethyl) pyrazol-1-yl]benzenesulfonamide dimethylacetamide monosolvate (2)

Crystal data

$\mathrm{C}_{17} \mathrm{H}_{14} \mathrm{~F}_{3} \mathrm{~N}_{3} \mathrm{O}_{2} \mathrm{~S} \cdot \mathrm{C}_{4} \mathrm{H}_{9} \mathrm{NO}$

$M_{r}=468.49$

Monoclinic, $P 2{ }_{1} / c$

$a=11.9584(3) \AA$

$b=9.2028(2) \AA$

$c=21.2811(6) \AA$

$\beta=103.3826(12)^{\circ}$

$V=2278.41(10) \AA^{3}$

$Z=4$

\section{Data collection}

Bruker D8-QUEST PHOTON-100 diffractometer

Radiation source: Incoatec $\mathrm{I} \mu \mathrm{S} \mathrm{Cu}$ microsource $\omega$ and $\varphi$-scans

Absorption correction: multi-scan

(SADABS; Bruker, 2016)

$T_{\min }=0.608, T_{\max }=0.753$

38939 measured reflections

\section{Refinement}

Refinement on $F^{2}$

Least-squares matrix: full

$R\left[F^{2}>2 \sigma\left(F^{2}\right)\right]=0.047$

$w R\left(F^{2}\right)=0.129$

$S=1.03$

4032 reflections

345 parameters

26 restraints

Primary atom site location: dual
$F(000)=976$

$D_{\mathrm{x}}=1.366 \mathrm{Mg} \mathrm{m}^{-3}$

$\mathrm{Cu} K \alpha$ radiation, $\lambda=1.54178 \AA$

Cell parameters from 9399 reflections

$\theta=3.8-66.8^{\circ}$

$\mu=1.75 \mathrm{~mm}^{-1}$

$T=298 \mathrm{~K}$

Block, colourless

$0.20 \times 0.18 \times 0.18 \mathrm{~mm}$

4032 independent reflections

3394 reflections with $I>2 \sigma(I)$

$R_{\text {int }}=0.041$

$\theta_{\text {max }}=66.9^{\circ}, \theta_{\min }=3.8^{\circ}$

$h=-14 \rightarrow 14$

$k=-10 \rightarrow 10$

$l=-25 \rightarrow 25$

Secondary atom site location: difference Fourier map

Hydrogen site location: mixed

$\mathrm{H}$ atoms treated by a mixture of independent and constrained refinement

$w=1 /\left[\sigma^{2}\left(F_{\mathrm{o}}^{2}\right)+(0.0707 P)^{2}+0.7443 P\right]$

where $P=\left(F_{\mathrm{o}}^{2}+2 F_{\mathrm{c}}^{2}\right) / 3$

$(\Delta / \sigma)_{\max }<0.001$

$\Delta \rho_{\text {max }}=0.22 \mathrm{e}^{-3}$

$\Delta \rho_{\min }=-0.28$ e $\AA^{-3}$ 


\section{Special details}

Geometry. All esds (except the esd in the dihedral angle between two 1.s. planes) are estimated using the full covariance matrix. The cell esds are taken into account individually in the estimation of esds in distances, angles and torsion angles; correlations between esds in cell parameters are only used when they are defined by crystal symmetry. An approximate (isotropic) treatment of cell esds is used for estimating esds involving l.s. planes.

Fractional atomic coordinates and isotropic or equivalent isotropic displacement parameters $\left(\AA^{2}\right)$

\begin{tabular}{|c|c|c|c|c|c|}
\hline & $x$ & $y$ & $z$ & $U_{\text {iso }} * / U_{\text {eq }}$ & Occ. $(<1)$ \\
\hline $\mathrm{S} 1$ & $0.43445(4)$ & $0.72988(6)$ & $0.57506(3)$ & 0.05785 (19) & \\
\hline $\mathrm{O} 1$ & $0.42323(14)$ & $0.6964(2)$ & $0.63856(8)$ & $0.0779(5)$ & \\
\hline $\mathrm{O} 2$ & $0.37684(12)$ & $0.85394(17)$ & $0.54250(9)$ & $0.0743(5)$ & \\
\hline N1 & $0.99144(14)$ & $0.8711(2)$ & $0.65110(9)$ & $0.0609(4)$ & \\
\hline $\mathrm{N} 2$ & $0.93377(13)$ & $0.81638(19)$ & $0.59372(8)$ & $0.0527(4)$ & \\
\hline N3 & $0.39037(16)$ & $0.5913(2)$ & $0.53096(11)$ & $0.0669(5)$ & \\
\hline $\mathrm{H} 1 \mathrm{~N}$ & $0.393(2)$ & $0.609(3)$ & $0.4914(6)$ & $0.083(9)^{*}$ & \\
\hline $\mathrm{H} 2 \mathrm{~N}$ & $0.424(2)$ & $0.515(2)$ & $0.5502(10)$ & $0.094(9)^{*}$ & \\
\hline $\mathrm{C} 1$ & $1.09752(17)$ & $0.8884(2)$ & $0.64353(11)$ & $0.0598(5)$ & \\
\hline $\mathrm{C} 2$ & $1.10928(16)$ & $0.8464(2)$ & $0.58289(11)$ & $0.0589(5)$ & \\
\hline $\mathrm{H} 2 \mathrm{~A}$ & 1.175547 & 0.850068 & 0.567120 & $0.071 *$ & \\
\hline $\mathrm{C} 3$ & $1.00221(15)$ & $0.7981(2)$ & $0.55086(10)$ & $0.0506(5)$ & \\
\hline $\mathrm{C} 4$ & $1.1872(2)$ & $0.9455(3)$ & 0.69911 (14) & $0.0815(7)$ & \\
\hline $\mathrm{F} 1$ & $1.2294(9)$ & $1.0652(10)$ & $0.6860(6)$ & $0.207(7)$ & $0.516(10)$ \\
\hline $\mathrm{F} 2$ & $1.2715(5)$ & $0.8604(7)$ & $0.7157(3)$ & $0.106(2)$ & $0.516(10)$ \\
\hline F3 & $1.1513(5)$ & $0.9639(15)$ & 0.7510 & $0.165(5)$ & $0.516(10)$ \\
\hline F1A & $1.2840(6)$ & $0.9624(18)$ & $0.6835(6)$ & $0.193(7)$ & $0.484(10)$ \\
\hline F2A & $1.2026(13)$ & $0.8676(12)$ & $0.7487(5)$ & $0.241(9)$ & $0.484(10)$ \\
\hline F3A & $1.1649(8)$ & $1.0709(8)$ & $0.7164(3)$ & $0.124(3)$ & $0.484(10)$ \\
\hline $\mathrm{C} 5$ & $0.96681(15)$ & $0.7310(2)$ & $0.48700(10)$ & $0.0502(5)$ & \\
\hline C6 & $0.88913(16)$ & $0.6162(2)$ & $0.47387(10)$ & $0.0534(5)$ & \\
\hline H6A & 0.854233 & 0.583021 & 0.505929 & $0.064^{*}$ & \\
\hline $\mathrm{C} 7$ & $0.86344(17)$ & $0.5516(2)$ & $0.41421(11)$ & $0.0599(5)$ & \\
\hline H7A & 0.810159 & 0.476258 & 0.406339 & $0.072 *$ & \\
\hline $\mathrm{C} 8$ & 0.91479 (19) & $0.5955(3)$ & $0.36545(11)$ & $0.0657(6)$ & \\
\hline $\mathrm{C} 9$ & $0.9924(2)$ & $0.7091(3)$ & $0.37893(12)$ & $0.0745(7)$ & \\
\hline H9A & 1.028377 & 0.740852 & 0.347052 & $0.089 *$ & \\
\hline $\mathrm{C} 10$ & $1.0178(2)$ & 0.7761 & $0.43806(12)$ & $0.0653(6)$ & \\
\hline $\mathrm{H} 10 \mathrm{~A}$ & 1.069851 & 0.852738 & 0.445453 & $0.078 *$ & \\
\hline $\mathrm{C} 11$ & $0.8864(3)$ & $0.5227(4)$ & $0.30007(14)$ & $0.1002(9)$ & \\
\hline H11A & 0.929940 & 0.567241 & 0.272607 & $0.150 *$ & \\
\hline H11B & 0.905441 & 0.421342 & 0.305008 & $0.150 *$ & \\
\hline $\mathrm{H} 11 \mathrm{C}$ & 0.805808 & 0.533181 & 0.281054 & $0.150^{*}$ & \\
\hline $\mathrm{C} 12$ & $0.81326(15)$ & $0.7913(2)$ & $0.58638(9)$ & $0.0491(4)$ & \\
\hline C13 & $0.77851(18)$ & $0.7144(3)$ & $0.63396(11)$ & $0.0626(6)$ & \\
\hline $\mathrm{H} 13 \mathrm{~A}$ & 0.832718 & 0.675882 & 0.668465 & $0.075^{*}$ & \\
\hline $\mathrm{C} 14$ & $0.66275(18)$ & 0.6949 & $0.63017(11)$ & $0.0620(6)$ & \\
\hline $\mathrm{H} 14 \mathrm{~A}$ & 0.638480 & 0.642754 & 0.662026 & $0.074 *$ & \\
\hline $\mathrm{C} 15$ & $0.58320(16)$ & $0.7530(2)$ & $0.57884(10)$ & $0.0497(5)$ & \\
\hline
\end{tabular}




$\begin{array}{llllll}\text { C16 } & 0.61849(16) & 0.8294(2) & 0.53087(10) & 0.0501(4) & \\ \text { H16A } & 0.564382 & 0.867533 & 0.496216 & 0.060^{*} & \\ \text { C17 } & 0.73436(16) & 0.8488(2) & 0.53465(10) & 0.0502(4) & \\ \text { H17A } & 0.758911 & 0.900046 & 0.502652 & 0.060^{*} & \\ \text { O1S } & 0.44913(16) & 0.63401(19) & 0.40777(9) & 0.0833(5) & 0.536(8) \\ \text { N1S } & 0.4762(4) & 0.7912(5) & 0.3367(2) & 0.0707(15) & 0.536(8) \\ \text { C1S } & 0.4097(4) & 0.7432(5) & 0.3738(2) & 0.0634(15) & 0.536(8) \\ \text { C2S } & 0.2989(2) & 0.8268(4) & 0.37048(15) & 0.0999(10) & 0.536(8) \\ \text { H1S } & 0.293223 & 0.904700 & 0.339905 & 0.150^{*} & 0.536(8) \\ \text { H2S } & 0.234527 & 0.762574 & 0.357160 & 0.150^{*} & 0.536(8) \\ \text { H3S } & 0.299015 & 0.865895 & 0.412316 & 0.150^{*} & 0.536(8) \\ \text { C3S } & 0.4369(3) & 0.9272(4) & 0.29637(16) & 0.1110(11) & 0.536(8) \\ \text { H4S } & 0.492151 & 0.951005 & 0.271683 & 0.167 * & 0.536(8) \\ \text { H5S } & 0.363604 & 0.909132 & 0.267630 & 0.167 * & 0.536(8) \\ \text { H6S } & 0.430222 & 1.006712 & 0.324457 & 0.167 * & 0.536(8) \\ \text { C4S } & 0.5805(3) & 0.7081(4) & 0.3364(2) & 0.1148(12) & 0.536(8) \\ \text { H7S } & 0.621272 & 0.753693 & 0.307813 & 0.172^{*} & 0.536(8) \\ \text { H8S } & 0.628586 & 0.705337 & 0.379286 & 0.172^{*} & 0.536(8) \\ \text { H9S } & 0.559878 & 0.610896 & 0.321895 & 0.172^{*} & 0.536(8) \\ \text { O1SA } & 0.44913(16) & 0.63401(19) & 0.40777(9) & 0.0833(5) & 0.464(8) \\ \text { N1SA } & 0.4034(4) & 0.8269(5) & 0.3446(2) & 0.0649(17) & 0.464(8) \\ \text { C1SA } & 0.4749(4) & 0.7183(6) & 0.3645(3) & 0.0628(17) & 0.464(8) \\ \text { C2SA } & 0.5805(3) & 0.7081(4) & 0.3364(2) & 0.1148(12) & 0.464(8) \\ \text { H10S } & 0.578131 & 0.784138 & 0.305225 & 0.172^{*} & 0.464(8) \\ \text { H11S } & 0.648469 & 0.718398 & 0.370407 & 0.172^{*} & 0.464(8) \\ \text { H12S } & 0.581462 & 0.615473 & 0.315826 & 0.172^{*} & 0.464(8) \\ \text { C3SA } & 0.4369(3) & 0.9272(4) & 0.29637(16) & 0.1110(11) & 0.464(8) \\ \text { H13S } & 0.380184 & 1.002320 & 0.284804 & 0.167 * & 0.464(8) \\ \text { H14S } & 0.510255 & 0.970064 & 0.314987 & 0.167 * & 0.464(8) \\ \text { H15S } & 0.441652 & 0.873150 & 0.258486 & 0.167 * & 0.464(8) \\ \text { C4SA } & 0.2989(2) & 0.8268(4) & 0.37048(15) & 0.0999(10) & 0.464(8) \\ \text { H16S } & 0.251945 & 0.908935 & 0.353710 & 0.150^{*} & 0.464(8) \\ \text { H17S } & 0.256497 & 0.738875 & 0.357782 & 0.150^{*} & 0.464(8) \\ \text { H18S } & 0.320425 & 0.832472 & 0.416762 & 0.150^{*} & 0.464(8) \\ & & & & & \\ & & & & \\ \end{array}$

Atomic displacement parameters $\left(\AA^{2}\right)$

\begin{tabular}{lllllll}
\hline & $U^{11}$ & $U^{22}$ & $U^{33}$ & $U^{12}$ & $U^{13}$ & $U^{23}$ \\
\hline S1 & $0.0393(3)$ & $0.0633(3)$ & $0.0779(4)$ & $0.0051(2)$ & $0.0279(2)$ & $0.0130(2)$ \\
O1 & $0.0628(9)$ & $0.1012(12)$ & $0.0821(11)$ & $0.0023(9)$ & $0.0422(8)$ & $0.0135(9)$ \\
O2 & $0.0459(8)$ & $0.0694(10)$ & $0.1140(13)$ & $0.0175(7)$ & $0.0316(8)$ & $0.0227(9)$ \\
N1 & $0.0464(9)$ & $0.0686(11)$ & $0.0664(11)$ & $-0.0031(8)$ & $0.0106(8)$ & $-0.0032(9)$ \\
N2 & $0.0359(8)$ & $0.0599(10)$ & $0.0626(10)$ & $-0.0017(7)$ & $0.0120(7)$ & $0.0023(8)$ \\
N3 & $0.0487(10)$ & $0.0682(12)$ & $0.0864(15)$ & $-0.0057(9)$ & $0.0207(10)$ & $0.0117(11)$ \\
C1 & $0.0424(11)$ & $0.0569(12)$ & $0.0785(14)$ & $-0.0031(9)$ & $0.0107(10)$ & $-0.0039(10)$ \\
C2 & $0.0363(10)$ & $0.0558(11)$ & $0.0861(15)$ & $-0.0016(8)$ & $0.0175(9)$ & $-0.0017(10)$ \\
C3 & $0.0376(9)$ & $0.0455(10)$ & $0.0708(12)$ & $0.0016(8)$ & $0.0167(9)$ & $0.0040(9)$ \\
C4 & $0.0555(14)$ & $0.0839(19)$ & $0.100(2)$ & $-0.0101(14)$ & $0.0084(13)$ & $-0.0165(16)$
\end{tabular}




\begin{tabular}{|c|c|c|c|c|c|c|}
\hline $\mathrm{F} 1$ & $0.159(9)$ & $0.135(7)$ & $0.260(12)$ & $-0.105(6)$ & $-0.090(7)$ & $0.070(7)$ \\
\hline $\mathrm{F} 2$ & $0.068(3)$ & $0.130(5)$ & $0.102(4)$ & $0.033(3)$ & $-0.020(2)$ & $-0.022(3)$ \\
\hline F3 & $0.080(3)$ & $0.289(13)$ & $0.130(6)$ & $-0.044(5)$ & $0.034(4)$ & $-0.126(8)$ \\
\hline F1A & $0.068(4)$ & $0.303(15)$ & $0.218(11)$ & $-0.082(6)$ & $0.057(5)$ & $-0.167(11)$ \\
\hline F2A & $0.273(15)$ & $0.216(10)$ & $0.146(9)$ & $-0.144(9)$ & $-0.133(9)$ & $0.112(8)$ \\
\hline F3A & $0.144(6)$ & $0.109(5)$ & $0.091(4)$ & $0.026(4)$ & -0.030 & -0.048 \\
\hline $\mathrm{C} 5$ & $0.0358(9)$ & $0.0483(10)$ & $0.0684(12)$ & $0.0056(8)$ & $0.0157(8)$ & $0.0052(9)$ \\
\hline C6 & $0.0414(10)$ & $0.0500(11)$ & 0.0717 (13) & $0.0019(8)$ & $0.0189(9)$ & $0.0050(9)$ \\
\hline $\mathrm{C} 7$ & $0.0447(10)$ & $0.0526(11)$ & $0.0816(14)$ & $0.0021(9)$ & $0.0128(10)$ & $-0.0020(10)$ \\
\hline C8 & $0.0521(12)$ & $0.0740(14)$ & $0.0701(14)$ & $0.0056(11)$ & $0.0122(10)$ & $-0.0017(11)$ \\
\hline C9 & $0.0664(14)$ & $0.0906(17)$ & $0.0732(15)$ & $-0.0061(13)$ & $0.0300(12)$ & $0.0042(13)$ \\
\hline $\mathrm{C} 10$ & $0.0552(12)$ & $0.0673(14)$ & $0.0776(15)$ & $-0.0114(10)$ & $0.0241(11)$ & $0.0021(11)$ \\
\hline $\mathrm{C} 11$ & $0.098(2)$ & $0.122(2)$ & $0.0806(18)$ & $-0.0092(19)$ & $0.0205(15)$ & $-0.0189(18)$ \\
\hline $\mathrm{C} 12$ & $0.0371(9)$ & $0.0512(10)$ & $0.0615(11)$ & $-0.0002(8)$ & $0.0163(8)$ & $0.0032(9)$ \\
\hline $\mathrm{C} 13$ & $0.0460(11)$ & $0.0772(14)$ & $0.0633(12)$ & $0.0024(10)$ & $0.0099(9)$ & $0.0218(11)$ \\
\hline $\mathrm{C} 14$ & $0.0494(11)$ & $0.0743(14)$ & $0.0662(13)$ & $-0.0018(10)$ & $0.0215(10)$ & $0.0220(11)$ \\
\hline $\mathrm{C} 15$ & $0.0387(9)$ & $0.0516(10)$ & $0.0630(12)$ & $0.0026(8)$ & $0.0204(8)$ & $0.0069(9)$ \\
\hline $\mathrm{C} 16$ & $0.0384(9)$ & $0.0534(11)$ & 0.0598 (11) & $0.0044(8)$ & $0.0141(8)$ & $0.0110(9)$ \\
\hline $\mathrm{C} 17$ & $0.0429(10)$ & $0.0512(10)$ & $0.0603(11)$ & $0.0014(8)$ & $0.0201(8)$ & $0.0102(9)$ \\
\hline O1S & $0.0909(12)$ & $0.0720(11)$ & $0.0897(12)$ & $0.0050(10)$ & $0.0262(10)$ & $0.0162(9)$ \\
\hline N1S & $0.070(3)$ & 0.069 & $0.074(3)$ & $-0.002(2)$ & $0.017(2)$ & $0.007(2)$ \\
\hline C1S & 0.058 & $0.064(3)$ & $0.065(3)$ & -0.001 & 0.007 (2) & -0.008 \\
\hline $\mathrm{C} 2 \mathrm{~S}$ & $0.0733(17)$ & $0.128(3)$ & $0.097(2)$ & $0.0294(18)$ & $0.0171(15)$ & $-0.0117(19)$ \\
\hline $\mathrm{C} 3 \mathrm{~S}$ & $0.146(3)$ & 0.0861 (19) & $0.091(2)$ & $-0.015(2)$ & $0.006(2)$ & $0.0259(17)$ \\
\hline $\mathrm{C} 4 \mathrm{~S}$ & $0.095(2)$ & $0.116(3)$ & $0.153(3)$ & $0.028(2)$ & $0.068(2)$ & 0.039 (2) \\
\hline O1SA & $0.0909(12)$ & $0.0720(11)$ & $0.0897(12)$ & $0.0050(10)$ & $0.0262(10)$ & $0.0162(9)$ \\
\hline N1SA & $0.064(3)$ & $0.061(3)$ & $0.064(3)$ & $0.007(2)$ & $0.003(2)$ & $0.000(2)$ \\
\hline C1SA & $0.060(3)$ & 0.059 (3) & 0.068 (4) & $-0.002(3)$ & 0.010 & 0.003 \\
\hline C2SA & $0.095(2)$ & $0.116(3)$ & $0.153(3)$ & $0.028(2)$ & $0.068(2)$ & 0.039 (2) \\
\hline C3SA & $0.146(3)$ & $0.0861(19)$ & $0.091(2)$ & $-0.015(2)$ & $0.006(2)$ & $0.0259(17)$ \\
\hline C4SA & $0.0733(17)$ & $0.128(3)$ & 0.097 (2) & $0.0294(18)$ & $0.0171(15)$ & $-0.0117(19)$ \\
\hline
\end{tabular}

Geometric parameters $\left(A,{ }^{\circ}\right)$

\begin{tabular}{llll}
\hline $\mathrm{S} 1-\mathrm{O} 1$ & $1.4223(16)$ & $\mathrm{C} 13-\mathrm{C} 14$ & $1.380(3)$ \\
$\mathrm{S} 1-\mathrm{O} 2$ & $1.4274(16)$ & $\mathrm{C} 13-\mathrm{H} 13 \mathrm{~A}$ & 0.9300 \\
$\mathrm{~S} 1-\mathrm{N} 3$ & $1.599(2)$ & $\mathrm{C} 14-\mathrm{C} 15$ & $1.380(3)$ \\
$\mathrm{S} 1-\mathrm{C} 15$ & $1.7747(19)$ & $\mathrm{C} 14-\mathrm{H} 14 \mathrm{~A}$ & 0.9300 \\
$\mathrm{~N} 1-\mathrm{C} 1$ & $1.325(3)$ & $\mathrm{C} 15-\mathrm{C} 16$ & $1.383(3)$ \\
$\mathrm{N} 1-\mathrm{N} 2$ & $1.353(2)$ & $\mathrm{C} 16-\mathrm{C} 17$ & $1.381(3)$ \\
$\mathrm{N} 2-\mathrm{C} 3$ & $1.370(2)$ & $\mathrm{C} 16-\mathrm{H} 16 \mathrm{~A}$ & 0.9300 \\
$\mathrm{~N} 2-\mathrm{C} 12$ & $1.432(2)$ & $\mathrm{C} 17-\mathrm{H} 17 \mathrm{~A}$ & 0.9300 \\
$\mathrm{~N} 3-\mathrm{H} 1 \mathrm{~N}$ & $0.864(9)$ & $\mathrm{O} 1 \mathrm{~S}-\mathrm{C} 1 \mathrm{~S}$ & $1.264(5)$ \\
$\mathrm{N} 3-\mathrm{H} 2 \mathrm{~N}$ & $0.865(9)$ & $\mathrm{N} 1 \mathrm{~S}-\mathrm{C} 1 \mathrm{~S}$ & $1.318(5)$ \\
$\mathrm{C} 1-\mathrm{C} 2$ & $1.385(3)$ & $\mathrm{N} 1 \mathrm{~S}-\mathrm{C} 4 \mathrm{~S}$ & $1.464(5)$ \\
$\mathrm{C} 1-\mathrm{C} 4$ & $1.496(3)$ & $\mathrm{N} 1 \mathrm{~S}-\mathrm{C} 3 \mathrm{~S}$ & $1.529(5)$ \\
$\mathrm{C} 2-\mathrm{C} 3$ & $1.378(3)$ & $\mathrm{C} 1 \mathrm{~S}-\mathrm{C} 2 \mathrm{~S}$ & $1.519(5)$ \\
$\mathrm{C} 2-\mathrm{H} 2 \mathrm{~A}$ & 0.9300 & $\mathrm{C} 2 \mathrm{~S}-\mathrm{H} 1 \mathrm{~S}$ & 0.9600
\end{tabular}




\begin{tabular}{|c|c|c|c|}
\hline $\mathrm{C} 3-\mathrm{C} 5$ & $1.463(3)$ & $\mathrm{C} 2 \mathrm{~S}-\mathrm{H} 2 \mathrm{~S}$ & 0.9600 \\
\hline $\mathrm{C} 4-\mathrm{F} 2 \mathrm{~A}$ & $1.254(5)$ & $\mathrm{C} 2 \mathrm{~S}-\mathrm{H} 3 \mathrm{~S}$ & 0.9600 \\
\hline $\mathrm{C} 4-\mathrm{F} 3 \mathrm{~A}$ & $1.258(5)$ & $\mathrm{C} 3 \mathrm{~S}-\mathrm{H} 4 \mathrm{~S}$ & 0.9600 \\
\hline $\mathrm{C} 4-\mathrm{F} 2$ & $1.261(4)$ & $\mathrm{C} 3 \mathrm{~S}-\mathrm{H} 5 \mathrm{~S}$ & 0.9600 \\
\hline $\mathrm{C} 4-\mathrm{F} 1$ & $1.270(5)$ & $\mathrm{C} 3 \mathrm{~S}-\mathrm{H} 6 \mathrm{~S}$ & 0.9600 \\
\hline $\mathrm{C} 4-\mathrm{F} 3$ & $1.285(6)$ & $\mathrm{C} 4 \mathrm{~S}-\mathrm{H} 7 \mathrm{~S}$ & 0.9600 \\
\hline $\mathrm{C} 4-\mathrm{F} 1 \mathrm{~A}$ & $1.286(5)$ & $\mathrm{C} 4 \mathrm{~S}-\mathrm{H} 8 \mathrm{~S}$ & 0.9600 \\
\hline $\mathrm{C} 5-\mathrm{C} 10$ & $1.386(3)$ & $\mathrm{C} 4 \mathrm{~S}-\mathrm{H} 9 \mathrm{~S}$ & 0.9600 \\
\hline $\mathrm{C} 5-\mathrm{C} 6$ & $1.392(3)$ & $\mathrm{O} 1 \mathrm{SA}-\mathrm{C} 1 \mathrm{SA}$ & $1.295(5)$ \\
\hline $\mathrm{C} 6-\mathrm{C} 7$ & $1.371(3)$ & N1SA-C1SA & $1.320(6)$ \\
\hline $\mathrm{C} 6-\mathrm{H} 6 \mathrm{~A}$ & 0.9300 & N1SA-C4SA & $1.477(5)$ \\
\hline $\mathrm{C} 7-\mathrm{C} 8$ & $1.383(3)$ & N1SA-C3SA & $1.502(5)$ \\
\hline C7-H7A & 0.9300 & $\mathrm{C} 1 \mathrm{SA}-\mathrm{C} 2 \mathrm{SA}$ & $1.519(5)$ \\
\hline $\mathrm{C} 8-\mathrm{C} 9$ & $1.384(3)$ & $\mathrm{C} 2 \mathrm{SA}-\mathrm{H} 10 \mathrm{~S}$ & 0.9600 \\
\hline $\mathrm{C} 8-\mathrm{C} 11$ & $1.510(4)$ & $\mathrm{C} 2 \mathrm{SA}-\mathrm{H} 11 \mathrm{~S}$ & 0.9600 \\
\hline $\mathrm{C} 9-\mathrm{C} 10$ & $1.371(3)$ & $\mathrm{C} 2 \mathrm{SA}-\mathrm{H} 12 \mathrm{~S}$ & 0.9600 \\
\hline C9-H9A & 0.9300 & $\mathrm{C} 3 \mathrm{SA}-\mathrm{H} 13 \mathrm{~S}$ & 0.9600 \\
\hline $\mathrm{C} 10-\mathrm{H} 10 \mathrm{~A}$ & 0.9300 & $\mathrm{C} 3 \mathrm{SA}-\mathrm{H} 14 \mathrm{~S}$ & 0.9600 \\
\hline C11-H11A & 0.9600 & $\mathrm{C} 3 \mathrm{SA}-\mathrm{H} 15 \mathrm{~S}$ & 0.9600 \\
\hline C11-H11B & 0.9600 & $\mathrm{C} 4 \mathrm{SA}-\mathrm{H} 16 \mathrm{~S}$ & 0.9600 \\
\hline $\mathrm{C} 11-\mathrm{H} 11 \mathrm{C}$ & 0.9600 & $\mathrm{C} 4 \mathrm{SA}-\mathrm{H} 17 \mathrm{~S}$ & 0.9600 \\
\hline $\mathrm{C} 12-\mathrm{C} 13$ & $1.376(3)$ & $\mathrm{C} 4 \mathrm{SA}-\mathrm{H} 18 \mathrm{~S}$ & 0.9600 \\
\hline $\mathrm{C} 12-\mathrm{C} 17$ & $1.379(3)$ & & \\
\hline $\mathrm{O} 1-\mathrm{S} 1-\mathrm{O} 2$ & $119.56(10)$ & $\mathrm{C} 12-\mathrm{C} 13-\mathrm{H} 13 \mathrm{~A}$ & 120.2 \\
\hline $\mathrm{O} 1-\mathrm{S} 1-\mathrm{N} 3$ & $107.11(11)$ & $\mathrm{C} 14-\mathrm{C} 13-\mathrm{H} 13 \mathrm{~A}$ & 120.2 \\
\hline $\mathrm{O} 2-\mathrm{S} 1-\mathrm{N} 3$ & $107.25(11)$ & $\mathrm{C} 13-\mathrm{C} 14-\mathrm{C} 15$ & $119.62(18)$ \\
\hline $\mathrm{O} 1-\mathrm{S} 1-\mathrm{C} 15$ & $107.28(10)$ & $\mathrm{C} 13-\mathrm{C} 14-\mathrm{H} 14 \mathrm{~A}$ & 120.2 \\
\hline $\mathrm{O} 2-\mathrm{S} 1-\mathrm{C} 15$ & $106.98(9)$ & $\mathrm{C} 15-\mathrm{C} 14-\mathrm{H} 14 \mathrm{~A}$ & 120.2 \\
\hline $\mathrm{N} 3-\mathrm{S} 1-\mathrm{C} 15$ & $108.24(9)$ & $\mathrm{C} 14-\mathrm{C} 15-\mathrm{C} 16$ & $120.60(17)$ \\
\hline $\mathrm{C} 1-\mathrm{N} 1-\mathrm{N} 2$ & $103.72(17)$ & $\mathrm{C} 14-\mathrm{C} 15-\mathrm{S} 1$ & $119.31(14)$ \\
\hline $\mathrm{N} 1-\mathrm{N} 2-\mathrm{C} 3$ & $112.86(15)$ & $\mathrm{C} 16-\mathrm{C} 15-\mathrm{S} 1$ & $120.09(15)$ \\
\hline $\mathrm{N} 1-\mathrm{N} 2-\mathrm{C} 12$ & $116.63(16)$ & $\mathrm{C} 17-\mathrm{C} 16-\mathrm{C} 15$ & $119.75(18)$ \\
\hline $\mathrm{C} 3-\mathrm{N} 2-\mathrm{C} 12$ & $130.47(17)$ & $\mathrm{C} 17-\mathrm{C} 16-\mathrm{H} 16 \mathrm{~A}$ & 120.1 \\
\hline $\mathrm{S} 1-\mathrm{N} 3-\mathrm{H} 1 \mathrm{~N}$ & $110.1(18)$ & $\mathrm{C} 15-\mathrm{C} 16-\mathrm{H} 16 \mathrm{~A}$ & 120.1 \\
\hline $\mathrm{S} 1-\mathrm{N} 3-\mathrm{H} 2 \mathrm{~N}$ & $108.5(19)$ & $\mathrm{C} 12-\mathrm{C} 17-\mathrm{C} 16$ & $119.27(17)$ \\
\hline $\mathrm{H} 1 \mathrm{~N}-\mathrm{N} 3-\mathrm{H} 2 \mathrm{~N}$ & $119.3(17)$ & $\mathrm{C} 12-\mathrm{C} 17-\mathrm{H} 17 \mathrm{~A}$ & 120.4 \\
\hline $\mathrm{N} 1-\mathrm{C} 1-\mathrm{C} 2$ & $112.74(19)$ & $\mathrm{C} 16-\mathrm{C} 17-\mathrm{H} 17 \mathrm{~A}$ & 120.4 \\
\hline $\mathrm{N} 1-\mathrm{C} 1-\mathrm{C} 4$ & $118.5(2)$ & $\mathrm{C} 1 \mathrm{~S}-\mathrm{N} 1 \mathrm{~S}-\mathrm{C} 4 \mathrm{~S}$ & $117.4(4)$ \\
\hline $\mathrm{C} 2-\mathrm{C} 1-\mathrm{C} 4$ & $128.7(2)$ & $\mathrm{C} 1 \mathrm{~S}-\mathrm{N} 1 \mathrm{~S}-\mathrm{C} 3 \mathrm{~S}$ & $117.4(4)$ \\
\hline $\mathrm{C} 3-\mathrm{C} 2-\mathrm{C} 1$ & $105.53(18)$ & $\mathrm{C} 4 \mathrm{~S}-\mathrm{N} 1 \mathrm{~S}-\mathrm{C} 3 \mathrm{~S}$ & $125.2(3)$ \\
\hline $\mathrm{C} 3-\mathrm{C} 2-\mathrm{H} 2 \mathrm{~A}$ & 127.2 & $\mathrm{O} 1 \mathrm{~S}-\mathrm{C} 1 \mathrm{~S}-\mathrm{N} 1 \mathrm{~S}$ & $114.3(4)$ \\
\hline $\mathrm{C} 1-\mathrm{C} 2-\mathrm{H} 2 \mathrm{~A}$ & 127.2 & $\mathrm{O} 1 \mathrm{~S}-\mathrm{C} 1 \mathrm{~S}-\mathrm{C} 2 \mathrm{~S}$ & $129.4(4)$ \\
\hline $\mathrm{N} 2-\mathrm{C} 3-\mathrm{C} 2$ & $105.14(18)$ & $\mathrm{N} 1 \mathrm{~S}-\mathrm{C} 1 \mathrm{~S}-\mathrm{C} 2 \mathrm{~S}$ & $116.3(4)$ \\
\hline $\mathrm{N} 2-\mathrm{C} 3-\mathrm{C} 5$ & $125.55(17)$ & $\mathrm{C} 1 \mathrm{~S}-\mathrm{C} 2 \mathrm{~S}-\mathrm{H} 1 \mathrm{~S}$ & 109.5 \\
\hline $\mathrm{C} 2-\mathrm{C} 3-\mathrm{C} 5$ & $129.17(18)$ & $\mathrm{C} 1 \mathrm{~S}-\mathrm{C} 2 \mathrm{~S}-\mathrm{H} 2 \mathrm{~S}$ & 109.5 \\
\hline $\mathrm{F} 2 \mathrm{~A}-\mathrm{C} 4-\mathrm{F} 3 \mathrm{~A}$ & $106.2(6)$ & $\mathrm{H} 1 \mathrm{~S}-\mathrm{C} 2 \mathrm{~S}-\mathrm{H} 2 \mathrm{~S}$ & 109.5 \\
\hline $\mathrm{F} 2-\mathrm{C} 4-\mathrm{F} 1$ & $105.5(4)$ & $\mathrm{C} 1 \mathrm{~S}-\mathrm{C} 2 \mathrm{~S}-\mathrm{H} 3 \mathrm{~S}$ & 109.5 \\
\hline
\end{tabular}




\begin{tabular}{|c|c|c|c|}
\hline $\mathrm{F} 2-\mathrm{C} 4-\mathrm{F} 3$ & $104.2(5)$ & $\mathrm{H} 1 \mathrm{~S}-\mathrm{C} 2 \mathrm{~S}-\mathrm{H} 3 \mathrm{~S}$ & 109.5 \\
\hline $\mathrm{F} 1-\mathrm{C} 4-\mathrm{F} 3$ & $107.7(6)$ & $\mathrm{H} 2 \mathrm{~S}-\mathrm{C} 2 \mathrm{~S}-\mathrm{H} 3 \mathrm{~S}$ & 109.5 \\
\hline $\mathrm{F} 2 \mathrm{~A}-\mathrm{C} 4-\mathrm{F} 1 \mathrm{~A}$ & $108.8(5)$ & $\mathrm{N} 1 \mathrm{~S}-\mathrm{C} 3 \mathrm{~S}-\mathrm{H} 4 \mathrm{~S}$ & 109.5 \\
\hline $\mathrm{F} 3 \mathrm{~A}-\mathrm{C} 4-\mathrm{F} 1 \mathrm{~A}$ & $103.2(5)$ & $\mathrm{N} 1 \mathrm{~S}-\mathrm{C} 3 \mathrm{~S}-\mathrm{H} 5 \mathrm{~S}$ & 109.5 \\
\hline $\mathrm{F} 2 \mathrm{~A}-\mathrm{C} 4-\mathrm{C} 1$ & $113.8(4)$ & $\mathrm{H} 4 \mathrm{~S}-\mathrm{C} 3 \mathrm{~S}-\mathrm{H} 5 \mathrm{~S}$ & 109.5 \\
\hline $\mathrm{F} 3 \mathrm{~A}-\mathrm{C} 4-\mathrm{C} 1$ & $113.0(3)$ & $\mathrm{N} 1 \mathrm{~S}-\mathrm{C} 3 \mathrm{~S}-\mathrm{H} 6 \mathrm{~S}$ & 109.5 \\
\hline $\mathrm{F} 2-\mathrm{C} 4-\mathrm{C} 1$ & $112.5(3)$ & $\mathrm{H} 4 \mathrm{~S}-\mathrm{C} 3 \mathrm{~S}-\mathrm{H} 6 \mathrm{~S}$ & 109.5 \\
\hline $\mathrm{F} 1-\mathrm{C} 4-\mathrm{C} 1$ & $112.3(4)$ & $\mathrm{H} 5 \mathrm{~S}-\mathrm{C} 3 \mathrm{~S}-\mathrm{H} 6 \mathrm{~S}$ & 109.5 \\
\hline $\mathrm{F} 3-\mathrm{C} 4-\mathrm{C} 1$ & $113.9(3)$ & $\mathrm{N} 1 \mathrm{~S}-\mathrm{C} 4 \mathrm{~S}-\mathrm{H} 7 \mathrm{~S}$ & 109.5 \\
\hline $\mathrm{F} 1 \mathrm{~A}-\mathrm{C} 4-\mathrm{C} 1$ & $111.2(4)$ & $\mathrm{N} 1 \mathrm{~S}-\mathrm{C} 4 \mathrm{~S}-\mathrm{H} 8 \mathrm{~S}$ & 109.5 \\
\hline $\mathrm{C} 10-\mathrm{C} 5-\mathrm{C} 6$ & $117.7(2)$ & $\mathrm{H} 7 \mathrm{~S}-\mathrm{C} 4 \mathrm{~S}-\mathrm{H} 8 \mathrm{~S}$ & 109.5 \\
\hline $\mathrm{C} 10-\mathrm{C} 5-\mathrm{C} 3$ & $119.25(18)$ & $\mathrm{N} 1 \mathrm{~S}-\mathrm{C} 4 \mathrm{~S}-\mathrm{H} 9 \mathrm{~S}$ & 109.5 \\
\hline $\mathrm{C} 6-\mathrm{C} 5-\mathrm{C} 3$ & $122.89(18)$ & $\mathrm{H} 7 \mathrm{~S}-\mathrm{C} 4 \mathrm{~S}-\mathrm{H} 9 \mathrm{~S}$ & 109.5 \\
\hline $\mathrm{C} 7-\mathrm{C} 6-\mathrm{C} 5$ & $120.77(19)$ & $\mathrm{H} 8 \mathrm{~S}-\mathrm{C} 4 \mathrm{~S}-\mathrm{H} 9 \mathrm{~S}$ & 109.5 \\
\hline $\mathrm{C} 7-\mathrm{C} 6-\mathrm{H} 6 \mathrm{~A}$ & 119.6 & $\mathrm{C} 1 \mathrm{SA}-\mathrm{N} 1 \mathrm{SA}-\mathrm{C} 4 \mathrm{SA}$ & $114.9(4)$ \\
\hline $\mathrm{C} 5-\mathrm{C} 6-\mathrm{H} 6 \mathrm{~A}$ & 119.6 & $\mathrm{C} 1 \mathrm{SA}-\mathrm{N} 1 \mathrm{SA}-\mathrm{C} 3 \mathrm{SA}$ & $115.4(4)$ \\
\hline $\mathrm{C} 6-\mathrm{C} 7-\mathrm{C} 8$ & $121.6(2)$ & $\mathrm{C} 4 \mathrm{SA}-\mathrm{N} 1 \mathrm{SA}-\mathrm{C} 3 \mathrm{SA}$ & $129.6(4)$ \\
\hline $\mathrm{C} 6-\mathrm{C} 7-\mathrm{H} 7 \mathrm{~A}$ & 119.2 & $\mathrm{O} 1 \mathrm{SA}-\mathrm{C} 1 \mathrm{SA}-\mathrm{N} 1 \mathrm{SA}$ & $116.0(4)$ \\
\hline $\mathrm{C} 8-\mathrm{C} 7-\mathrm{H} 7 \mathrm{~A}$ & 119.2 & $\mathrm{O} 1 \mathrm{SA}-\mathrm{C} 1 \mathrm{SA}-\mathrm{C} 2 \mathrm{SA}$ & $126.8(4)$ \\
\hline $\mathrm{C} 7-\mathrm{C} 8-\mathrm{C} 9$ & $117.3(2)$ & $\mathrm{N} 1 \mathrm{SA}-\mathrm{C} 1 \mathrm{SA}-\mathrm{C} 2 \mathrm{SA}$ & $117.2(4)$ \\
\hline $\mathrm{C} 7-\mathrm{C} 8-\mathrm{C} 11$ & $121.1(2)$ & $\mathrm{C} 1 \mathrm{SA}-\mathrm{C} 2 \mathrm{SA}-\mathrm{H} 10 \mathrm{~S}$ & 109.5 \\
\hline $\mathrm{C} 9-\mathrm{C} 8-\mathrm{C} 11$ & $121.5(2)$ & $\mathrm{C} 1 \mathrm{SA}-\mathrm{C} 2 \mathrm{SA}-\mathrm{H} 11 \mathrm{~S}$ & 109.5 \\
\hline $\mathrm{C} 10-\mathrm{C} 9-\mathrm{C} 8$ & $121.7(2)$ & $\mathrm{H} 10 \mathrm{~S}-\mathrm{C} 2 \mathrm{SA}-\mathrm{H} 11 \mathrm{~S}$ & 109.5 \\
\hline $\mathrm{C} 10-\mathrm{C} 9-\mathrm{H} 9 \mathrm{~A}$ & 119.2 & $\mathrm{C} 1 \mathrm{SA}-\mathrm{C} 2 \mathrm{SA}-\mathrm{H} 12 \mathrm{~S}$ & 109.5 \\
\hline $\mathrm{C} 8-\mathrm{C} 9-\mathrm{H} 9 \mathrm{~A}$ & 119.2 & $\mathrm{H} 10 \mathrm{~S}-\mathrm{C} 2 \mathrm{SA}-\mathrm{H} 12 \mathrm{~S}$ & 109.5 \\
\hline $\mathrm{C} 9-\mathrm{C} 10-\mathrm{C} 5$ & $120.9(2)$ & $\mathrm{H} 11 \mathrm{~S}-\mathrm{C} 2 \mathrm{SA}-\mathrm{H} 12 \mathrm{~S}$ & 109.5 \\
\hline $\mathrm{C} 9-\mathrm{C} 10-\mathrm{H} 10 \mathrm{~A}$ & 119.6 & N1SA $-\mathrm{C} 3 \mathrm{SA}-\mathrm{H} 13 \mathrm{~S}$ & 109.5 \\
\hline $\mathrm{C} 5-\mathrm{C} 10-\mathrm{H} 10 \mathrm{~A}$ & 119.6 & $\mathrm{~N} 1 \mathrm{SA}-\mathrm{C} 3 \mathrm{SA}-\mathrm{H} 14 \mathrm{~S}$ & 109.5 \\
\hline $\mathrm{C} 8-\mathrm{C} 11-\mathrm{H} 11 \mathrm{~A}$ & 109.5 & $\mathrm{H} 13 \mathrm{~S}-\mathrm{C} 3 \mathrm{SA}-\mathrm{H} 14 \mathrm{~S}$ & 109.5 \\
\hline $\mathrm{C} 8-\mathrm{C} 11-\mathrm{H} 11 \mathrm{~B}$ & 109.5 & $\mathrm{~N} 1 \mathrm{SA}-\mathrm{C} 3 \mathrm{SA}-\mathrm{H} 15 \mathrm{~S}$ & 109.5 \\
\hline $\mathrm{H} 11 \mathrm{~A}-\mathrm{C} 11-\mathrm{H} 11 \mathrm{~B}$ & 109.5 & $\mathrm{H} 13 \mathrm{~S}-\mathrm{C} 3 \mathrm{SA}-\mathrm{H} 15 \mathrm{~S}$ & 109.5 \\
\hline $\mathrm{C} 8-\mathrm{C} 11-\mathrm{H} 11 \mathrm{C}$ & 109.5 & $\mathrm{H} 14 \mathrm{~S}-\mathrm{C} 3 \mathrm{SA}-\mathrm{H} 15 \mathrm{~S}$ & 109.5 \\
\hline $\mathrm{H} 11 \mathrm{~A}-\mathrm{C} 11-\mathrm{H} 11 \mathrm{C}$ & 109.5 & $\mathrm{~N} 1 \mathrm{SA}-\mathrm{C} 4 \mathrm{SA}-\mathrm{H} 16 \mathrm{~S}$ & 109.5 \\
\hline $\mathrm{H} 11 \mathrm{~B}-\mathrm{C} 11-\mathrm{H} 11 \mathrm{C}$ & 109.5 & $\mathrm{~N} 1 \mathrm{SA}-\mathrm{C} 4 \mathrm{SA}-\mathrm{H} 17 \mathrm{~S}$ & 109.5 \\
\hline $\mathrm{C} 13-\mathrm{C} 12-\mathrm{C} 17$ & $121.16(17)$ & $\mathrm{H} 16 \mathrm{~S}-\mathrm{C} 4 \mathrm{SA}-\mathrm{H} 17 \mathrm{~S}$ & 109.5 \\
\hline $\mathrm{C} 13-\mathrm{C} 12-\mathrm{N} 2$ & $118.05(17)$ & $\mathrm{N} 1 \mathrm{SA}-\mathrm{C} 4 \mathrm{SA}-\mathrm{H} 18 \mathrm{~S}$ & 109.5 \\
\hline $\mathrm{C} 17-\mathrm{C} 12-\mathrm{N} 2$ & $120.72(16)$ & $\mathrm{H} 16 \mathrm{~S}-\mathrm{C} 4 \mathrm{SA}-\mathrm{H} 18 \mathrm{~S}$ & 109.5 \\
\hline $\mathrm{C} 12-\mathrm{C} 13-\mathrm{C} 14$ & $119.60(19)$ & $\mathrm{H} 17 \mathrm{~S}-\mathrm{C} 4 \mathrm{SA}-\mathrm{H} 18 \mathrm{~S}$ & 109.5 \\
\hline $\mathrm{C} 1-\mathrm{N} 1-\mathrm{N} 2-\mathrm{C} 3$ & $0.5(2)$ & $\mathrm{C} 7-\mathrm{C} 8-\mathrm{C} 9-\mathrm{C} 10$ & $-0.1(4)$ \\
\hline $\mathrm{C} 1-\mathrm{N} 1-\mathrm{N} 2-\mathrm{C} 12$ & $-177.59(17)$ & $\mathrm{C} 11-\mathrm{C} 8-\mathrm{C} 9-\mathrm{C} 10$ & $179.5(3)$ \\
\hline $\mathrm{N} 2-\mathrm{N} 1-\mathrm{C} 1-\mathrm{C} 2$ & $0.1(2)$ & $\mathrm{C} 8-\mathrm{C} 9-\mathrm{C} 10-\mathrm{C} 5$ & $0.6(4)$ \\
\hline $\mathrm{N} 2-\mathrm{N} 1-\mathrm{C} 1-\mathrm{C} 4$ & $-178.8(2)$ & $\mathrm{C} 6-\mathrm{C} 5-\mathrm{C} 10-\mathrm{C} 9$ & $-0.2(3)$ \\
\hline $\mathrm{N} 1-\mathrm{C} 1-\mathrm{C} 2-\mathrm{C} 3$ & $-0.6(3)$ & $\mathrm{C} 3-\mathrm{C} 5-\mathrm{C} 10-\mathrm{C} 9$ & $175.6(2)$ \\
\hline $\mathrm{C} 4-\mathrm{C} 1-\mathrm{C} 2-\mathrm{C} 3$ & $178.1(2)$ & $\mathrm{N} 1-\mathrm{N} 2-\mathrm{C} 12-\mathrm{C} 13$ & $-50.1(3)$ \\
\hline $\mathrm{N} 1-\mathrm{N} 2-\mathrm{C} 3-\mathrm{C} 2$ & $-0.9(2)$ & $\mathrm{C} 3-\mathrm{N} 2-\mathrm{C} 12-\mathrm{C} 13$ & $132.2(2)$ \\
\hline $\mathrm{C} 12-\mathrm{N} 2-\mathrm{C} 3-\mathrm{C} 2$ & $176.86(19)$ & $\mathrm{N} 1-\mathrm{N} 2-\mathrm{C} 12-\mathrm{C} 17$ & $126.8(2)$ \\
\hline $\mathrm{N} 1-\mathrm{N} 2-\mathrm{C} 3-\mathrm{C} 5$ & $175.06(18)$ & $\mathrm{C} 3-\mathrm{N} 2-\mathrm{C} 12-\mathrm{C} 17$ & $-50.9(3)$ \\
\hline
\end{tabular}




$\begin{array}{llll}\mathrm{C} 12-\mathrm{N} 2-\mathrm{C} 3-\mathrm{C} 5 & -7.2(3) & \mathrm{C} 17-\mathrm{C} 12-\mathrm{C} 13-\mathrm{C} 14 & -0.3(3) \\ \mathrm{C} 1-\mathrm{C} 2-\mathrm{C} 3-\mathrm{N} 2 & 0.9(2) & \mathrm{N} 2-\mathrm{C} 12-\mathrm{C} 13-\mathrm{C} 14 & 176.6(2) \\ \mathrm{C} 1-\mathrm{C} 2-\mathrm{C} 3-\mathrm{C} 5 & -174.9(2) & \mathrm{C} 12-\mathrm{C} 13-\mathrm{C} 14-\mathrm{C} 15 & -0.2(4) \\ \mathrm{N} 1-\mathrm{C} 1-\mathrm{C} 4-\mathrm{F} 2 \mathrm{~A} & 59.7(11) & \mathrm{C} 13-\mathrm{C} 14-\mathrm{C} 15-\mathrm{C} 16 & 0.7(3) \\ \mathrm{C} 2-\mathrm{C} 1-\mathrm{C} 4-\mathrm{F} 2 \mathrm{~A} & -119.0(11) & \mathrm{C} 13-\mathrm{C} 14-\mathrm{C} 15-\mathrm{S} 1 & -178.95(18) \\ \mathrm{N} 1-\mathrm{C} 1-\mathrm{C} 4-\mathrm{F} 3 \mathrm{~A} & -61.5(7) & \mathrm{O} 1-\mathrm{S} 1-\mathrm{C} 15-\mathrm{C} 14 & 20.1(2) \\ \mathrm{C} 2-\mathrm{C} 1-\mathrm{C} 4-\mathrm{F} 3 \mathrm{~A} & 119.8(7) & \mathrm{O} 2-\mathrm{S} 1-\mathrm{C} 15-\mathrm{C} 14 & 149.50(18) \\ \mathrm{N} 1-\mathrm{C} 1-\mathrm{C} 4-\mathrm{F} 2 & 122.4(5) & \mathrm{N} 3-\mathrm{S} 1-\mathrm{C} 15-\mathrm{C} 14 & -95.2(2) \\ \mathrm{C} 2-\mathrm{C} 1-\mathrm{C} 4-\mathrm{F} 2 & -56.3(6) & \mathrm{O} 1-\mathrm{S} 1-\mathrm{C} 15-\mathrm{C} 16 & -159.56(17) \\ \mathrm{N} 1-\mathrm{C} 1-\mathrm{C} 4-\mathrm{F} 1 & -118.7(9) & \mathrm{O} 2-\mathrm{S} 1-\mathrm{C} 15-\mathrm{C} 16 & -30.12(19) \\ \mathrm{C} 2-\mathrm{C} 1-\mathrm{C} 4-\mathrm{F} 1 & 62.6(9) & \mathrm{N} 3-\mathrm{S} 1-\mathrm{C} 15-\mathrm{C} 16 & 85.17(18) \\ \mathrm{N} 1-\mathrm{C} 1-\mathrm{C} 4-\mathrm{F} 3 & 4.0(8) & \mathrm{C} 14-\mathrm{C} 15-\mathrm{C} 16-\mathrm{C} 17 & -0.6(3) \\ \mathrm{C} 2-\mathrm{C} 1-\mathrm{C} 4-\mathrm{F} 3 & -174.7(8) & \mathrm{S} 1-\mathrm{C} 15-\mathrm{C} 16-\mathrm{C} 17 & 179.03(15) \\ \mathrm{N} 1-\mathrm{C} 1-\mathrm{C} 4-\mathrm{F} 1 \mathrm{~A} & -177.1(9) & \mathrm{C} 13-\mathrm{C} 12-\mathrm{C} 17-\mathrm{C} 16 & 0.4(3) \\ \mathrm{C} 2-\mathrm{C} 1-\mathrm{C} 4-\mathrm{F} 1 \mathrm{~A} & 4.2(10) & \mathrm{N} 2-\mathrm{C} 12-\mathrm{C} 17-\mathrm{C} 16 & -176.44(18) \\ \mathrm{N} 2-\mathrm{C} 3-\mathrm{C} 5-\mathrm{C} 10 & 149.9(2) & \mathrm{C} 15-\mathrm{C} 16-\mathrm{C} 17-\mathrm{C} 12 & 0.0(3) \\ \mathrm{C} 2-\mathrm{C} 3-\mathrm{C} 5-\mathrm{C} 10 & -35.1(3) & \mathrm{C} 4 \mathrm{~S}-\mathrm{N} 1 \mathrm{~S}-\mathrm{C} 1 \mathrm{~S}-\mathrm{O} 1 \mathrm{~S} & 4.0(6) \\ \mathrm{N} 2-\mathrm{C} 3-\mathrm{C} 5-\mathrm{C} 6 & -34.5(3) & \mathrm{C} 3 \mathrm{~S}-\mathrm{N} 1 \mathrm{~S}-\mathrm{C} 1 \mathrm{~S}-\mathrm{O} 1 \mathrm{~S} & -177.4(3) \\ \mathrm{C} 2-\mathrm{C} 3-\mathrm{C} 5-\mathrm{C} 6 & 140.5(2) & \mathrm{C} 4 \mathrm{~S}-\mathrm{N} 1 \mathrm{~S}-\mathrm{C} 1 \mathrm{~S}-\mathrm{C} 2 \mathrm{~S} & -176.8(4) \\ \mathrm{C} 10-\mathrm{C} 5-\mathrm{C} 6-\mathrm{C} 7 & -0.7(3) & \mathrm{C} 3 \mathrm{~S}-\mathrm{N} 1 \mathrm{~S}-\mathrm{C} 1 \mathrm{C}-\mathrm{C} 2 \mathrm{~S} & 1.8(6) \\ \mathrm{C} 3-\mathrm{C} 5-\mathrm{C} 6-\mathrm{C} 7 & -176.33(18) & \mathrm{C} 4 \mathrm{SA}-\mathrm{N} 1 \mathrm{SA}-\mathrm{C} 1 \mathrm{SA}-\mathrm{O} 1 \mathrm{SA} & -5.6(6) \\ \mathrm{C} 5-\mathrm{C} 6-\mathrm{C} 7-\mathrm{C} 8 & 1.2(3) & \mathrm{C} 3 \mathrm{SA}-\mathrm{N} 1 \mathrm{SA}-\mathrm{C} 1 \mathrm{SA}-\mathrm{O} 1 \mathrm{SA} & 177.7(4) \\ \mathrm{C} 6-\mathrm{C} 7-\mathrm{C} 8-\mathrm{C} 9 & -0.8(3) & \mathrm{C} 4 \mathrm{SA}-\mathrm{N} 1 \mathrm{SA}-\mathrm{C} 1 \mathrm{SA}-\mathrm{C} 2 \mathrm{SA} & 176.6(4) \\ \mathrm{C} 6-\mathrm{C} 7-\mathrm{C} 8-\mathrm{C} 11 & 179.6(2) & \mathrm{C} 3 \mathrm{SA}-\mathrm{N} 1 \mathrm{SA}-\mathrm{C} 1 \mathrm{SA}-\mathrm{C} 2 \mathrm{SA} & -0.1(6) \\ & & & \end{array}$

Hydrogen-bond geometry $\left(A,{ }^{\circ}\right)$

\begin{tabular}{lllll}
\hline$D-\mathrm{H} \cdots A$ & $D-\mathrm{H}$ & $\mathrm{H} \cdots A$ & $D \cdots A$ & $D-\mathrm{H} \cdots A$ \\
\hline $\mathrm{N} 3-\mathrm{H} 1 N \cdots \mathrm{O} 1 \mathrm{Sa}$ & $0.86(1)$ & $2.06(1)$ & $2.894(3)$ & $163(2)$ \\
$\mathrm{N} 3-\mathrm{H} 2 N \cdots \mathrm{O} 1 \mathrm{Sa}^{\mathrm{i}}$ & $0.87(1)$ & $2.08(1)$ & $2.920(3)$ & $162(3)$ \\
\hline
\end{tabular}

Symmetry code: (i) $-x+1,-y+1,-z+1$.

4-[5-(4-Methylphenyl)-3-(trifluoromethyl)pyrazol-1-yl]benzenesulfonamide N-methylpyrrolidin-2-one monosolvate (3)

Crystal data

$\mathrm{C}_{17} \mathrm{H}_{14} \mathrm{~F}_{3} \mathrm{~N}_{3} \mathrm{O}_{2} \mathrm{~S} \cdot \mathrm{C}_{5} \mathrm{H}_{9} \mathrm{NO}$

$M_{r}=480.50$

Monoclinic, $P 2_{1} / c$ $a=11.9978(4) \AA$

$b=9.0896(3) \AA$

$c=21.9732(8) \AA$

$\beta=101.358(2)^{\circ}$

$V=2349.36(14) \AA^{3}$

$Z=4$
$F(000)=1000$

$D_{\mathrm{x}}=1.358 \mathrm{Mg} \mathrm{m}^{-3}$

$\mathrm{Cu} K \alpha$ radiation, $\lambda=1.54178 \AA$

Cell parameters from 9878 reflections

$\theta=3.8-66.5^{\circ}$

$\mu=1.71 \mathrm{~mm}^{-1}$

$T=298 \mathrm{~K}$

Block, colourless

$0.20 \times 0.18 \times 0.18 \mathrm{~mm}$ 


\section{Data collection}

Bruker D8-QUEST PHOTON-100 diffractometer

Radiation source: Incoatec $\mathrm{I} \mu \mathrm{S} \mathrm{Cu}$ microsource $\omega$ and $\varphi$-scans

Absorption correction: multi-scan

(SADABS; Bruker, 2016)

$T_{\min }=0.593, T_{\max }=0.753$

24378 measured reflections

\section{Refinement}

Refinement on $F^{2}$

Least-squares matrix: full

$R\left[F^{2}>2 \sigma\left(F^{2}\right)\right]=0.048$

$w R\left(F^{2}\right)=0.136$

$S=1.03$

4146 reflections

389 parameters

103 restraints

Primary atom site location: dual

Secondary atom site location: difference Fourier map
4146 independent reflections

3112 reflections with $I>2 \sigma(I)$

$R_{\text {int }}=0.047$

$\theta_{\text {max }}=66.8^{\circ}, \theta_{\min }=3.8^{\circ}$

$h=-14 \rightarrow 14$

$k=-10 \rightarrow 9$

$l=-24 \rightarrow 25$

Hydrogen site location: mixed

$\mathrm{H}$ atoms treated by a mixture of independent and constrained refinement

$w=1 /\left[\sigma^{2}\left(F_{\mathrm{o}}^{2}\right)+(0.0683 P)^{2}+0.6367 P\right]$ where $P=\left(F_{\mathrm{o}}^{2}+2 F_{\mathrm{c}}{ }^{2}\right) / 3$

$(\Delta / \sigma)_{\max }<0.001$

$\Delta \rho_{\max }=0.22 \mathrm{e}^{-3}$

$\Delta \rho_{\min }=-0.24$ e $\AA^{-3}$

Extinction correction: SHELXL2018

(Sheldrick, 2015b)

Extinction coefficient: 0.0093 (5)

Special details

Geometry. All esds (except the esd in the dihedral angle between two 1.s. planes) are estimated using the full covariance matrix. The cell esds are taken into account individually in the estimation of esds in distances, angles and torsion angles; correlations between esds in cell parameters are only used when they are defined by crystal symmetry. An approximate (isotropic) treatment of cell esds is used for estimating esds involving 1.s. planes.

Fractional atomic coordinates and isotropic or equivalent isotropic displacement parameters $\left(\AA^{2}\right)$

\begin{tabular}{llllll}
\hline & $x$ & $y$ & $z$ & $U_{\text {iso }} * / U_{\text {eq }}$ & Occ. $(<1)$ \\
\hline S1 & $0.42443(5)$ & $0.72995(8)$ & $0.57323(3)$ & $0.0743(3)$ & \\
O1 & $0.40662(15)$ & $0.6918(3)$ & $0.63344(9)$ & $0.0970(6)$ & \\
O2 & $0.37053(14)$ & $0.8572(2)$ & $0.54265(10)$ & $0.0974(6)$ & \\
N1 & $0.97465(15)$ & $0.8705(2)$ & $0.65213(9)$ & $0.0718(5)$ & \\
N2 & $0.92235(13)$ & $0.8146(2)$ & $0.59662(8)$ & $0.0600(5)$ & \\
N3 & $0.38435(16)$ & $0.5916(3)$ & $0.52861(10)$ & $0.0796(6)$ & \\
H1N & $0.393(3)$ & $0.614(3)$ & $0.4911(7)$ & $0.119^{*}$ & \\
H2N & $0.421(2)$ & $0.514(2)$ & $0.5457(12)$ & $0.119^{*}$ & \\
C1 & $1.08126(19)$ & $0.8883(3)$ & $0.64533(12)$ & $0.0728(6)$ & \\
C2 & $1.09811(18)$ & $0.8453(3)$ & $0.58771(12)$ & $0.0687(6)$ & \\
H2A & 1.165852 & 0.848585 & 0.573206 & $0.082 *$ & \\
C3 & $0.99450(16)$ & $0.7967(2)$ & $0.55607(10)$ & $0.0565(5)$ & \\
C4 & $1.1659(3)$ & $0.9471(4)$ & $0.69872(16)$ & $0.1011(10)$ & \\
F1 & $1.2535(9)$ & $0.994(2)$ & $0.6765(5)$ & $0.222(8)$ & $0.507(13)$ \\
F3 & $1.1276(7)$ & $1.0600(9)$ & $0.7205(3)$ & $0.134(3)$ & $0.507(13)$ \\
F2 & $1.1967(14)$ & $0.8585(8)$ & $0.7407(5)$ & $0.232(9)$ & $0.507(13)$ \\
F1A & $1.1958(8)$ & $1.0844(6)$ & $0.6935(7)$ & $0.182(7)$ & $0.493(13)$ \\
F2A & $1.2593(4)$ & $0.8749(8)$ & $0.7101(3)$ & $0.108(3)$ & $0.493(13)$ \\
F3A & $1.1318(6)$ & $0.9433(19)$ & $0.7522(4)$ & $0.194(8)$ & $0.493(13)$
\end{tabular}




\begin{tabular}{|c|c|c|c|c|c|}
\hline $\mathrm{C} 5$ & $0.96376(16)$ & $0.7310(2)$ & $0.49422(10)$ & $0.0560(5)$ & \\
\hline C6 & $0.88572(16)$ & $0.6174(2)$ & $0.48013(11)$ & $0.0590(5)$ & \\
\hline H6A & 0.848432 & 0.582550 & 0.510538 & $0.071^{*}$ & \\
\hline $\mathrm{C} 7$ & $0.86273(17)$ & $0.5559(3)$ & $0.42205(11)$ & $0.0653(6)$ & \\
\hline H7A & 0.809584 & 0.480362 & 0.413807 & $0.078^{*}$ & \\
\hline $\mathrm{C} 8$ & $0.9163(2)$ & $0.6029(3)$ & $0.37558(12)$ & $0.0733(6)$ & \\
\hline C9 & $0.9950(2)$ & $0.7151(3)$ & $0.38973(13)$ & $0.0833(8)$ & \\
\hline H9A & 1.033100 & 0.748272 & 0.359382 & $0.100^{*}$ & \\
\hline $\mathrm{C} 10$ & $1.0182(2)$ & $0.7789(3)$ & $0.44766(12)$ & $0.0733(7)$ & \\
\hline H10A & 1.070912 & 0.854952 & 0.455685 & $0.088^{*}$ & \\
\hline $\mathrm{C} 11$ & $0.8899(3)$ & $0.5348(4)$ & $0.31160(14)$ & $0.1128(11)$ & \\
\hline H11A & 0.935098 & 0.581327 & 0.285594 & $0.169^{*}$ & \\
\hline H11B & 0.907036 & 0.431568 & 0.314617 & $0.169^{*}$ & \\
\hline $\mathrm{H} 11 \mathrm{C}$ & 0.810760 & 0.548188 & 0.293943 & $0.169^{*}$ & \\
\hline $\mathrm{C} 12$ & $0.80263(16)$ & $0.7899(2)$ & $0.58845(10)$ & $0.0561(5)$ & \\
\hline $\mathrm{C} 13$ & $0.76268(19)$ & $0.7091(3)$ & $0.63267(11)$ & $0.0715(6)$ & \\
\hline $\mathrm{H} 13 \mathrm{~A}$ & 0.813363 & 0.667305 & 0.665627 & $0.086^{*}$ & \\
\hline C14 & $0.64752(19)$ & $0.6904(3)$ & $0.62788(11)$ & $0.0740(7)$ & \\
\hline H14A & 0.620015 & 0.635728 & 0.657536 & $0.089^{*}$ & \\
\hline $\mathrm{C} 15$ & $0.57292(17)$ & $0.7531(2)$ & $0.57887(11)$ & $0.0615(6)$ & \\
\hline $\mathrm{C} 16$ & $0.61338(17)$ & $0.8324(2)$ & $0.53439(10)$ & $0.0597(5)$ & \\
\hline H16A & 0.562852 & 0.873081 & 0.501098 & $0.072 *$ & \\
\hline $\mathrm{C} 17$ & $0.72897(16)$ & $0.8514(2)$ & $0.53925(10)$ & $0.0582(5)$ & \\
\hline H17A & 0.756691 & 0.905349 & 0.509478 & $0.070^{*}$ & \\
\hline O1S & $0.46487(18)$ & $0.6396(2)$ & $0.41492(10)$ & $0.0977(6)$ & $0.679(8)$ \\
\hline N1S & $0.3890(4)$ & $0.8101(5)$ & $0.34400(18)$ & $0.0848(14)$ & $0.679(8)$ \\
\hline C1S & $0.4695(5)$ & $0.7240(8)$ & $0.3717(3)$ & $0.0727(16)$ & $0.679(8)$ \\
\hline $\mathrm{C} 2 \mathrm{~S}$ & $0.5661(7)$ & $0.7546(11)$ & $0.3406(4)$ & $0.122(3)$ & $0.679(8)$ \\
\hline $\mathrm{H} 2 \mathrm{~S} 1$ & 0.624984 & 0.807865 & 0.368541 & $0.146^{*}$ & $0.679(8)$ \\
\hline $\mathrm{H} 2 \mathrm{~S} 2$ & 0.598100 & 0.662814 & 0.329412 & $0.146^{*}$ & $0.679(8)$ \\
\hline $\mathrm{C} 3 \mathrm{~S}$ & $0.5277(6)$ & $0.8373(13)$ & $0.2880(4)$ & $0.177(4)$ & $0.679(8)$ \\
\hline $\mathrm{H} 3 \mathrm{~S} 1$ & 0.520389 & 0.776555 & 0.251096 & $0.212 *$ & $0.679(8)$ \\
\hline H3S2 & 0.580123 & 0.916926 & 0.284945 & $0.212 *$ & $0.679(8)$ \\
\hline $\mathrm{C} 4 \mathrm{~S}$ & $0.4020(6)$ & $0.9032(9)$ & $0.2952(4)$ & $0.117(3)$ & $0.679(8)$ \\
\hline $\mathrm{H} 4 \mathrm{~S} 1$ & 0.405189 & 1.006227 & 0.306865 & $0.140^{*}$ & $0.679(8)$ \\
\hline $\mathrm{H} 4 \mathrm{~S} 2$ & 0.343951 & 0.887895 & 0.258223 & $0.140 *$ & $0.679(8)$ \\
\hline $\mathrm{C} 5 \mathrm{~S}$ & $0.2769(6)$ & $0.8127(10)$ & $0.3636(4)$ & $0.144(3)$ & $0.679(8)$ \\
\hline $\mathrm{H} 5 \mathrm{~S} 1$ & 0.227545 & 0.881874 & 0.338547 & $0.216^{*}$ & $0.679(8)$ \\
\hline H5S2 & 0.243357 & 0.716451 & 0.358569 & $0.216^{*}$ & $0.679(8)$ \\
\hline H5S3 & 0.287600 & 0.841352 & 0.406450 & $0.216^{*}$ & $0.679(8)$ \\
\hline O1SA & $0.46487(18)$ & $0.6396(2)$ & $0.41492(10)$ & $0.0977(6)$ & $0.321(8)$ \\
\hline N1SA & $0.4849(10)$ & $0.7734(12)$ & $0.3361(5)$ & $0.129(4)$ & $0.321(8)$ \\
\hline C1SA & $0.4263(10)$ & $0.7305(16)$ & $0.3763(6)$ & $0.086(5)$ & $0.321(8)$ \\
\hline C2SA & $0.3286(12)$ & $0.8325(19)$ & $0.3689(8)$ & $0.127(6)$ & $0.321(8)$ \\
\hline $\mathrm{H} 2 \mathrm{~S} 3$ & 0.257860 & 0.777722 & 0.361922 & $0.152^{*}$ & $0.321(8)$ \\
\hline $\mathrm{H} 2 \mathrm{~S} 4$ & 0.332888 & 0.891552 & 0.406092 & $0.152 *$ & $0.321(8)$ \\
\hline C3SA & $0.3331(13)$ & 0.9204 (19) & $0.3193(8)$ & $0.182(8)$ & $0.321(8)$ \\
\hline H3S3 & 0.326878 & 1.022754 & 0.330697 & $0.218^{*}$ & $0.321(8)$ \\
\hline
\end{tabular}




\begin{tabular}{llllll} 
H3S4 & 0.270203 & 0.897681 & 0.285595 & $0.218^{*}$ & $0.321(8)$ \\
C4SA & $0.4571(13)$ & $0.8927(16)$ & $0.2963(7)$ & $0.096(4)$ & $0.321(8)$ \\
H4S3 & 0.447513 & 0.866133 & 0.252777 & $0.116^{*}$ & $0.321(8)$ \\
H4S4 & 0.509558 & 0.974392 & 0.305894 & $0.116^{*}$ & $0.321(8)$ \\
C5SA & $0.5910(16)$ & $0.693(2)$ & $0.3311(11)$ & $0.151(8)$ & $0.321(8)$ \\
H5S4 & 0.624693 & 0.737444 & 0.299464 & $0.227^{*}$ & $0.321(8)$ \\
H5S5 & 0.643550 & 0.697079 & 0.370117 & $0.227^{*}$ & $0.321(8)$ \\
H5S6 & 0.573025 & 0.591953 & 0.320419 & $0.227^{*}$ & $0.321(8)$ \\
\hline
\end{tabular}

Atomic displacement parameters $\left(\AA^{2}\right)$

\begin{tabular}{|c|c|c|c|c|c|c|}
\hline & $U^{11}$ & $U^{22}$ & $U^{33}$ & $U^{12}$ & $U^{13}$ & $U^{23}$ \\
\hline S1 & $0.0452(3)$ & $0.0899(5)$ & $0.0951(5)$ & $0.0062(3)$ & 0.0318 & $0.0201(3)$ \\
\hline $\mathrm{O} 1$ & $0.0704(11)$ & $0.1386(17)$ & $0.0948(12)$ & $0.0002(11)$ & $0.0471(9)$ & $0.0159(12)$ \\
\hline $\mathrm{O} 2$ & $0.0537(9)$ & $0.0935(13)$ & $0.1515(17)$ & $0.0230(9)$ & $0.0360(10)$ & $0.0376(12)$ \\
\hline N1 & $0.0572(11)$ & $0.0795(14)$ & $0.0772(12)$ & $-0.0030(9)$ & $0.0096(9)$ & $-0.0089(10)$ \\
\hline N2 & $0.0441(9)$ & $0.0647(11)$ & $0.0712(11)$ & $-0.0021(8)$ & $0.0115(8)$ & $-0.0004(9)$ \\
\hline N3 & $0.0570(11)$ & $0.0913(16)$ & $0.0933(15)$ & $-0.0123(10)$ & $0.0214(11)$ & $0.0146(12)$ \\
\hline $\mathrm{C} 1$ & $0.0527(13)$ & $0.0696(15)$ & $0.0930(17)$ & $-0.0038(11)$ & $0.0070(11)$ & $-0.0111(13)$ \\
\hline $\mathrm{C} 2$ & $0.0451(11)$ & $0.0639(14)$ & $0.0979(17)$ & $-0.0047(10)$ & $0.0157(11)$ & $-0.0069(13)$ \\
\hline $\mathrm{C} 3$ & $0.0431(10)$ & $0.0484(11)$ & $0.0788(14)$ & $0.0005(9)$ & $0.0137(9)$ & $0.0035(10)$ \\
\hline $\mathrm{C} 4$ & $0.075(2)$ & $0.109(3)$ & $0.116(3)$ & $-0.0200(19)$ & $0.0116(18)$ & $-0.032(2)$ \\
\hline F1 & $0.134(6)$ & $0.34(2)$ & $0.207(8)$ & $-0.145(9)$ & $0.058(6)$ & $-0.142(11)$ \\
\hline F3 & $0.170(6)$ & $0.118(5)$ & $0.092(4)$ & $-0.016(4)$ & $-0.024(3)$ & $-0.040(3)$ \\
\hline F2 & $0.257(16)$ & $0.166(7)$ & $0.194(13)$ & $-0.029(9)$ & $-0.151(12)$ & $0.050(8)$ \\
\hline F1A & $0.141(8)$ & $0.079(4)$ & $0.273(14)$ & $-0.039(4)$ & $-0.092(8)$ & $0.003(5)$ \\
\hline F2A & $0.060(3)$ & $0.127(5)$ & $0.123(5)$ & $0.016(3)$ & $-0.017(2)$ & $-0.022(4)$ \\
\hline F3A & $0.099(5)$ & $0.37(2)$ & $0.115(6)$ & $-0.071(7)$ & $0.028(4)$ & $-0.102(10)$ \\
\hline $\mathrm{C} 5$ & $0.0396(10)$ & $0.0501(11)$ & $0.0794(14)$ & $0.0040(8)$ & $0.0148(9)$ & $0.0049(10)$ \\
\hline C6 & $0.0464(11)$ & $0.0529(12)$ & $0.0806(14)$ & $-0.0008(9)$ & $0.0196(10)$ & $0.0030(11)$ \\
\hline $\mathrm{C} 7$ & $0.0496(12)$ & $0.0584(13)$ & $0.0879(16)$ & $-0.0026(10)$ & $0.0134(11)$ & $-0.0041(12)$ \\
\hline $\mathrm{C} 8$ & $0.0614(13)$ & $0.0784(16)$ & $0.0801(15)$ & $0.0018(12)$ & $0.0143(11)$ & $-0.0048(13)$ \\
\hline C9 & $0.0771(16)$ & $0.097(2)$ & $0.0826(17)$ & -0.0118 & $0.0327(13)$ & $0.0055(15)$ \\
\hline $\mathrm{C} 10$ & $0.0592(13)$ & $0.0724(15)$ & $0.0925(18)$ & $-0.0155(11)$ & $0.0254(12)$ & $0.0022(13)$ \\
\hline C11 & $0.116(2)$ & $0.131(3)$ & $0.093(2)$ & $-0.018(2)$ & $0.0238(18)$ & $-0.024(2)$ \\
\hline $\mathrm{C} 12$ & $0.0432(10)$ & $0.0579(12)$ & $0.0692(13)$ & $0.0010(9)$ & $0.0162(9)$ & $0.0030(10)$ \\
\hline $\mathrm{C} 13$ & $0.0549(13)$ & $0.0855(17)$ & $0.0734(14)$ & $0.0030(11)$ & $0.0111(10)$ & $0.0234(13)$ \\
\hline C14 & $0.0591(13)$ & $0.0916(18)$ & $0.0756(15)$ & $-0.0006(12)$ & $0.0239(11)$ & $0.0270(13)$ \\
\hline $\mathrm{C} 15$ & $0.0459(11)$ & $0.0686(14)$ & $0.0750(14)$ & $0.0044(10)$ & $0.0242(10)$ & $0.0100(11)$ \\
\hline $\mathrm{C} 16$ & $0.0471(11)$ & $0.0610(13)$ & $0.0733(13)$ & $0.0056(9)$ & $0.0176(9)$ & $0.0131(11)$ \\
\hline $\mathrm{C} 17$ & $0.0489(11)$ & $0.0581(13)$ & $0.0712(13)$ & $0.0009(9)$ & $0.0205(9)$ & $0.0117(10)$ \\
\hline O1S & $0.0985(14)$ & $0.1014(15)$ & $0.0988(13)$ & $0.0077(12)$ & $0.0332(11)$ & $0.0285(12)$ \\
\hline N1S & $0.095(3)$ & $0.075(3)$ & $0.077(2)$ & $-0.005(2)$ & $-0.001(2)$ & $0.005(2)$ \\
\hline $\mathrm{C} 1 \mathrm{~S}$ & $0.065(3)$ & 0.083 & $0.069(3)$ & $-0.010(3)$ & $0.011(2)$ & $0.003(3)$ \\
\hline $\mathrm{C} 2 \mathrm{~S}$ & $0.100(4)$ & $0.154(6)$ & $0.120(4)$ & $-0.013(5)$ & $0.044(4)$ & $0.032(5)$ \\
\hline $\mathrm{C} 3 \mathrm{~S}$ & $0.116(5)$ & $0.270(8)$ & $0.140(5)$ & $-0.050(5)$ & $0.014(4)$ & $0.093(5)$ \\
\hline $\mathrm{C} 4 \mathrm{~S}$ & $0.118(6)$ & $0.109(5)$ & $0.112(4)$ & $-0.008(4)$ & $-0.005(4)$ & $0.021(4)$ \\
\hline $\mathrm{C} 5 \mathrm{~S}$ & $0.082(4)$ & $0.169(7)$ & $0.185(7)$ & 0.015 & $0.037(4)$ & $-0.061(5)$ \\
\hline
\end{tabular}




\begin{tabular}{lllllll} 
O1SA & $0.0985(14)$ & $0.1014(15)$ & $0.0988(13)$ & $0.0077(12)$ & $0.0332(11)$ & $0.0285(12)$ \\
N1SA & $0.143(9)$ & $0.119(7)$ & $0.129(8)$ & $-0.005(7)$ & $0.038(7)$ & $0.011(6)$ \\
C1SA & $0.090(9)$ & $0.072(6)$ & $0.095(8)$ & $0.006(7)$ & $0.011(6)$ & $-0.008(6)$ \\
C2SA & $0.122(10)$ & $0.136(9)$ & $0.112(8)$ & $0.010(8)$ & $-0.003(8)$ & $-0.021(7)$ \\
C3SA & $0.189(11)$ & $0.170(11)$ & $0.174(11)$ & $0.010(9)$ & $0.007(8)$ & $-0.009(9)$ \\
C4SA & $0.128(9)$ & $0.078(6)$ & $0.080(6)$ & $0.041(7)$ & $0.011(7)$ & $0.030(5)$ \\
C5SA & $0.158(13)$ & $0.129(11)$ & $0.193(14)$ & $0.051(9)$ & $0.099(10)$ & $0.031(10)$ \\
\hline
\end{tabular}

Geometric parameters $\left(\stackrel{A}{\circ}{ }^{o}\right)$

\begin{tabular}{|c|c|c|c|}
\hline $\mathrm{S} 1-\mathrm{O} 1$ & $1.4240(18)$ & $\mathrm{C} 14-\mathrm{C} 15$ & $1.381(3)$ \\
\hline $\mathrm{S} 1-\mathrm{O} 2$ & $1.4271(18)$ & $\mathrm{C} 14-\mathrm{H} 14 \mathrm{~A}$ & 0.9300 \\
\hline $\mathrm{S} 1-\mathrm{N} 3$ & $1.609(2)$ & $\mathrm{C} 15-\mathrm{C} 16$ & $1.377(3)$ \\
\hline $\mathrm{S} 1-\mathrm{C} 15$ & $1.774(2)$ & $\mathrm{C} 16-\mathrm{C} 17$ & $1.381(3)$ \\
\hline $\mathrm{N} 1-\mathrm{C} 1$ & $1.327(3)$ & $\mathrm{C} 16-\mathrm{H} 16 \mathrm{~A}$ & 0.9300 \\
\hline $\mathrm{N} 1-\mathrm{N} 2$ & $1.356(2)$ & $\mathrm{C} 17-\mathrm{H} 17 \mathrm{~A}$ & 0.9300 \\
\hline $\mathrm{N} 2-\mathrm{C} 3$ & $1.369(3)$ & $\mathrm{O} 1 \mathrm{~S}-\mathrm{C} 1 \mathrm{~S}$ & $1.230(5)$ \\
\hline $\mathrm{N} 2-\mathrm{C} 12$ & $1.430(2)$ & $\mathrm{N} 1 \mathrm{~S}-\mathrm{C} 1 \mathrm{~S}$ & $1.297(6)$ \\
\hline $\mathrm{N} 3-\mathrm{H} 1 \mathrm{~N}$ & $0.875(10)$ & $\mathrm{N} 1 \mathrm{~S}-\mathrm{C} 4 \mathrm{~S}$ & $1.399(7)$ \\
\hline $\mathrm{N} 3-\mathrm{H} 2 \mathrm{~N}$ & $0.875(10)$ & $\mathrm{N} 1 \mathrm{~S}-\mathrm{C} 5 \mathrm{~S}$ & $1.492(8)$ \\
\hline $\mathrm{C} 1-\mathrm{C} 2$ & $1.377(3)$ & $\mathrm{C} 1 \mathrm{~S}-\mathrm{C} 2 \mathrm{~S}$ & $1.483(9)$ \\
\hline $\mathrm{C} 1-\mathrm{C} 4$ & $1.492(4)$ & $\mathrm{C} 2 \mathrm{~S}-\mathrm{C} 3 \mathrm{~S}$ & $1.379(11)$ \\
\hline $\mathrm{C} 2-\mathrm{C} 3$ & $1.372(3)$ & $\mathrm{C} 2 \mathrm{~S}-\mathrm{H} 2 \mathrm{~S} 1$ & 0.9700 \\
\hline $\mathrm{C} 2-\mathrm{H} 2 \mathrm{~A}$ & 0.9300 & $\mathrm{C} 2 \mathrm{~S}-\mathrm{H} 2 \mathrm{~S} 2$ & 0.9700 \\
\hline $\mathrm{C} 3-\mathrm{C} 5$ & $1.464(3)$ & $\mathrm{C} 3 \mathrm{~S}-\mathrm{C} 4 \mathrm{~S}$ & $1.659(11)$ \\
\hline $\mathrm{C} 4-\mathrm{F} 2$ & $1.226(6)$ & $\mathrm{C} 3 \mathrm{~S}-\mathrm{H} 3 \mathrm{~S} 1$ & 0.9700 \\
\hline $\mathrm{C} 4-\mathrm{F} 3$ & $1.258(5)$ & $\mathrm{C} 3 \mathrm{~S}-\mathrm{H} 3 \mathrm{~S} 2$ & 0.9700 \\
\hline $\mathrm{C} 4-\mathrm{F} 2 \mathrm{~A}$ & $1.279(5)$ & $\mathrm{C} 4 \mathrm{~S}-\mathrm{H} 4 \mathrm{~S} 1$ & 0.9700 \\
\hline $\mathrm{C} 4-\mathrm{F} 1 \mathrm{~A}$ & $1.310(6)$ & $\mathrm{C} 4 \mathrm{~S}-\mathrm{H} 4 \mathrm{~S} 2$ & 0.9700 \\
\hline $\mathrm{C} 4-\mathrm{F} 1$ & $1.315(6)$ & $\mathrm{C} 5 \mathrm{~S}-\mathrm{H} 5 \mathrm{~S} 1$ & 0.9600 \\
\hline $\mathrm{C} 4-\mathrm{F} 3 \mathrm{~A}$ & $1.320(7)$ & $\mathrm{C} 5 \mathrm{~S}-\mathrm{H} 5 \mathrm{~S} 2$ & 0.9600 \\
\hline $\mathrm{C} 5-\mathrm{C} 6$ & $1.387(3)$ & $\mathrm{C} 5 \mathrm{~S}-\mathrm{H} 5 \mathrm{~S} 3$ & 0.9600 \\
\hline $\mathrm{C} 5-\mathrm{C} 10$ & $1.388(3)$ & $\mathrm{O} 1 \mathrm{SA}-\mathrm{C} 1 \mathrm{SA}$ & $1.209(8)$ \\
\hline $\mathrm{C} 6-\mathrm{C} 7$ & $1.371(3)$ & $\mathrm{N} 1 \mathrm{SA}-\mathrm{C} 1 \mathrm{SA}$ & $1.293(9)$ \\
\hline C6-H6A & 0.9300 & $\mathrm{~N} 1 \mathrm{SA}-\mathrm{C} 4 \mathrm{SA}$ & $1.392(9)$ \\
\hline $\mathrm{C} 7-\mathrm{C} 8$ & $1.378(3)$ & $\mathrm{N} 1 \mathrm{SA}-\mathrm{C} 5 \mathrm{SA}$ & $1.492(11)$ \\
\hline C7-H7A & 0.9300 & $\mathrm{C} 1 \mathrm{SA}-\mathrm{C} 2 \mathrm{SA}$ & $1.478(11)$ \\
\hline $\mathrm{C} 8-\mathrm{C} 9$ & $1.383(4)$ & $\mathrm{C} 2 \mathrm{SA}-\mathrm{C} 3 \mathrm{SA}$ & $1.361(14)$ \\
\hline $\mathrm{C} 8-\mathrm{C} 11$ & $1.512(4)$ & $\mathrm{C} 2 \mathrm{SA}-\mathrm{H} 2 \mathrm{~S} 3$ & 0.9700 \\
\hline $\mathrm{C} 9-\mathrm{C} 10$ & $1.376(4)$ & $\mathrm{C} 2 \mathrm{SA}-\mathrm{H} 2 \mathrm{~S} 4$ & 0.9700 \\
\hline C9-H9A & 0.9300 & $\mathrm{C} 3 \mathrm{SA}-\mathrm{C} 4 \mathrm{SA}$ & $1.682(14)$ \\
\hline $\mathrm{C} 10-\mathrm{H} 10 \mathrm{~A}$ & 0.9300 & $\mathrm{C} 3 \mathrm{SA}-\mathrm{H} 3 \mathrm{~S} 3$ & 0.9700 \\
\hline $\mathrm{C} 11-\mathrm{H} 11 \mathrm{~A}$ & 0.9600 & $\mathrm{C} 3 \mathrm{SA}-\mathrm{H} 3 \mathrm{~S} 4$ & 0.9700 \\
\hline C11-H11B & 0.9600 & $\mathrm{C} 4 \mathrm{SA}-\mathrm{H} 4 \mathrm{~S} 3$ & 0.9700 \\
\hline $\mathrm{C} 11-\mathrm{H} 11 \mathrm{C}$ & 0.9600 & $\mathrm{C} 4 \mathrm{SA}-\mathrm{H} 4 \mathrm{~S} 4$ & 0.9700 \\
\hline $\mathrm{C} 12-\mathrm{C} 17$ & $1.373(3)$ & $\mathrm{C} 5 \mathrm{SA}-\mathrm{H} 5 \mathrm{~S} 4$ & 0.9600 \\
\hline $\mathrm{C} 12-\mathrm{C} 13$ & $1.377(3)$ & C5SA-H5S5 & 0.9600 \\
\hline $\mathrm{C} 13-\mathrm{C} 14$ & $1.375(3)$ & C5SA-H5S6 & 0.9600 \\
\hline
\end{tabular}




$$
\text { C13-H13A }
$$

$\mathrm{O} 1-\mathrm{S} 1-\mathrm{O} 2$

$\mathrm{O} 1-\mathrm{S} 1-\mathrm{N} 3$

$\mathrm{O} 2-\mathrm{S} 1-\mathrm{N} 3$

$\mathrm{O} 1-\mathrm{S} 1-\mathrm{C} 15$

$\mathrm{O} 2-\mathrm{S} 1-\mathrm{C} 15$

N3-S1-C15

$\mathrm{C} 1-\mathrm{N} 1-\mathrm{N} 2$

$\mathrm{N} 1-\mathrm{N} 2-\mathrm{C} 3$

$\mathrm{N} 1-\mathrm{N} 2-\mathrm{C} 12$

$\mathrm{C} 3-\mathrm{N} 2-\mathrm{C} 12$

$\mathrm{S} 1-\mathrm{N} 3-\mathrm{H} 1 \mathrm{~N}$

$\mathrm{S} 1-\mathrm{N} 3-\mathrm{H} 2 \mathrm{~N}$

$\mathrm{H} 1 \mathrm{~N}-\mathrm{N} 3-\mathrm{H} 2 \mathrm{~N}$

$\mathrm{N} 1-\mathrm{C} 1-\mathrm{C} 2$

$\mathrm{N} 1-\mathrm{C} 1-\mathrm{C} 4$

$\mathrm{C} 2-\mathrm{C} 1-\mathrm{C} 4$

$\mathrm{C} 3-\mathrm{C} 2-\mathrm{C} 1$

$\mathrm{C} 3-\mathrm{C} 2-\mathrm{H} 2 \mathrm{~A}$

$\mathrm{C} 1-\mathrm{C} 2-\mathrm{H} 2 \mathrm{~A}$

$\mathrm{N} 2-\mathrm{C} 3-\mathrm{C} 2$

$\mathrm{N} 2-\mathrm{C} 3-\mathrm{C} 5$

$\mathrm{C} 2-\mathrm{C} 3-\mathrm{C} 5$

$\mathrm{F} 2-\mathrm{C} 4-\mathrm{F} 3$

$\mathrm{F} 2 \mathrm{~A}-\mathrm{C} 4-\mathrm{F} 1 \mathrm{~A}$

$\mathrm{F} 2-\mathrm{C} 4-\mathrm{F} 1$

$\mathrm{F} 3-\mathrm{C} 4-\mathrm{F} 1$

$\mathrm{F} 2 \mathrm{~A}-\mathrm{C} 4-\mathrm{F} 3 \mathrm{~A}$

$\mathrm{F} 1 \mathrm{~A}-\mathrm{C} 4-\mathrm{F} 3 \mathrm{~A}$

$\mathrm{F} 2-\mathrm{C} 4-\mathrm{C} 1$

F3-C4-C1

$\mathrm{F} 2 \mathrm{~A}-\mathrm{C} 4-\mathrm{C} 1$

$\mathrm{F} 1 \mathrm{~A}-\mathrm{C} 4-\mathrm{C} 1$

$\mathrm{F} 1-\mathrm{C} 4-\mathrm{C} 1$

$\mathrm{F} 3 \mathrm{~A}-\mathrm{C} 4-\mathrm{C} 1$

C6- 5 - $-\mathrm{C} 10$

$\mathrm{C} 6-\mathrm{C} 5-\mathrm{C} 3$

$\mathrm{C} 10-\mathrm{C} 5-\mathrm{C} 3$

$\mathrm{C} 7-\mathrm{C} 6-\mathrm{C} 5$

$\mathrm{C} 7-\mathrm{C} 6-\mathrm{H} 6 \mathrm{~A}$

$\mathrm{C} 5-\mathrm{C} 6-\mathrm{H} 6 \mathrm{~A}$

$\mathrm{C} 6-\mathrm{C} 7-\mathrm{C} 8$

$\mathrm{C} 6-\mathrm{C} 7-\mathrm{H} 7 \mathrm{~A}$

$\mathrm{C} 8-\mathrm{C} 7-\mathrm{H} 7 \mathrm{~A}$

C7-C8-C9

$\mathrm{C} 7-\mathrm{C} 8-\mathrm{C} 11$

$\mathrm{C} 9-\mathrm{C} 8-\mathrm{C} 11$
0.9300

$119.86(12)$

$107.11(12)$

107.05 (13)

107.24 (11)

$107.02(10)$

$108.11(11)$

$103.29(18)$

$112.96(16)$

116.57 (17)

130.41 (18)

109 (2)

108 (2)

116.4 (17)

112.9 (2)

118.5 (2)

$128.6(2)$

$105.9(2)$

127.0

127.0

105.0 (2)

$125.23(18)$

129.7 (2)

109.1 (6)

$105.2(4)$

$110.5(6)$

104.7 (6)

$103.0(6)$

$104.0(6)$

114.5 (4)

110.4 (4)

113.9 (4)

$115.4(5)$

$107.2(4)$

114.1 (4)

$117.6(2)$

$123.13(19)$

119.17 (19)

$121.0(2)$

119.5

119.5

121.7 (2)

119.1

119.1

117.4 (2)

$121.3(2)$

$121.3(2)$
$\mathrm{C} 16-\mathrm{C} 15-\mathrm{S} 1$

C14-C15-S1

C15-C16-C17

C15-C16-H16A

C17-C16-H16A

$\mathrm{C} 12-\mathrm{C} 17-\mathrm{C} 16$

C12-C17-H17A

C16-C17-H17A

C1S-N1S-C4S

$\mathrm{C} 1 \mathrm{~S}-\mathrm{N} 1 \mathrm{~S}-\mathrm{C} 5 \mathrm{~S}$

$\mathrm{C} 4 \mathrm{~S}-\mathrm{N} 1 \mathrm{~S}-\mathrm{C} 5 \mathrm{~S}$

O1S-C1S-N1S

$\mathrm{O} 1 \mathrm{~S}-\mathrm{C} 1 \mathrm{~S}-\mathrm{C} 2 \mathrm{~S}$

$\mathrm{N} 1 \mathrm{~S}-\mathrm{C} 1 \mathrm{~S}-\mathrm{C} 2 \mathrm{~S}$

$\mathrm{C} 3 \mathrm{~S}-\mathrm{C} 2 \mathrm{~S}-\mathrm{C} 1 \mathrm{~S}$

$\mathrm{C} 3 \mathrm{~S}-\mathrm{C} 2 \mathrm{~S}-\mathrm{H} 2 \mathrm{~S} 1$

$\mathrm{C} 1 \mathrm{~S}-\mathrm{C} 2 \mathrm{~S}-\mathrm{H} 2 \mathrm{~S} 1$

$\mathrm{C} 3 \mathrm{~S}-\mathrm{C} 2 \mathrm{~S}-\mathrm{H} 2 \mathrm{~S} 2$

$\mathrm{C} 1 \mathrm{~S}-\mathrm{C} 2 \mathrm{~S}-\mathrm{H} 2 \mathrm{~S} 2$

$\mathrm{H} 2 \mathrm{~S} 1-\mathrm{C} 2 \mathrm{~S}-\mathrm{H} 2 \mathrm{~S} 2$

$\mathrm{C} 2 \mathrm{~S}-\mathrm{C} 3 \mathrm{~S}-\mathrm{C} 4 \mathrm{~S}$

$\mathrm{C} 2 \mathrm{~S}-\mathrm{C} 3 \mathrm{~S}-\mathrm{H} 3 \mathrm{~S} 1$

$\mathrm{C} 4 \mathrm{~S}-\mathrm{C} 3 \mathrm{~S}-\mathrm{H} 3 \mathrm{~S} 1$

$\mathrm{C} 2 \mathrm{~S}-\mathrm{C} 3 \mathrm{~S}-\mathrm{H} 3 \mathrm{~S} 2$

$\mathrm{C} 4 \mathrm{~S}-\mathrm{C} 3 \mathrm{~S}-\mathrm{H} 3 \mathrm{~S} 2$

$\mathrm{H} 3 \mathrm{~S} 1-\mathrm{C} 3 \mathrm{~S}-\mathrm{H} 3 \mathrm{~S} 2$

$\mathrm{N} 1 \mathrm{~S}-\mathrm{C} 4 \mathrm{~S}-\mathrm{C} 3 \mathrm{~S}$

$\mathrm{N} 1 \mathrm{~S}-\mathrm{C} 4 \mathrm{~S}-\mathrm{H} 4 \mathrm{~S} 1$

$\mathrm{C} 3 \mathrm{~S}-\mathrm{C} 4 \mathrm{~S}-\mathrm{H} 4 \mathrm{~S} 1$

$\mathrm{N} 1 \mathrm{~S}-\mathrm{C} 4 \mathrm{~S}-\mathrm{H} 4 \mathrm{~S} 2$

$\mathrm{C} 3 \mathrm{~S}-\mathrm{C} 4 \mathrm{~S}-\mathrm{H} 4 \mathrm{~S} 2$

$\mathrm{H} 4 \mathrm{~S} 1-\mathrm{C} 4 \mathrm{~S}-\mathrm{H} 4 \mathrm{~S} 2$

$\mathrm{N} 1 \mathrm{~S}-\mathrm{C} 5 \mathrm{~S}-\mathrm{H} 5 \mathrm{~S} 1$

$\mathrm{N} 1 \mathrm{~S}-\mathrm{C} 5 \mathrm{~S}-\mathrm{H} 5 \mathrm{~S} 2$

$\mathrm{H} 5 \mathrm{~S} 1-\mathrm{C} 5 \mathrm{~S}-\mathrm{H} 5 \mathrm{~S} 2$

$\mathrm{N} 1 \mathrm{~S}-\mathrm{C} 5 \mathrm{~S}-\mathrm{H} 5 \mathrm{~S} 3$

$\mathrm{H} 5 \mathrm{~S} 1-\mathrm{C} 5 \mathrm{~S}-\mathrm{H} 5 \mathrm{~S} 3$

$\mathrm{H} 5 \mathrm{~S} 2-\mathrm{C} 5 \mathrm{~S}-\mathrm{H} 5 \mathrm{~S} 3$

C1SA-N1SA-C4SA

C1SA-N1SA-C5SA

C4SA-N1SA-C5SA

O1SA-C1SA-N1SA

O1SA-C1SA-C2SA

N1SA-C1SA-C2SA

C3SA-C2SA-C1SA

$\mathrm{C} 3 \mathrm{SA}-\mathrm{C} 2 \mathrm{SA}-\mathrm{H} 2 \mathrm{~S} 3$
$120.12(17)$

119.57 (16)

120.0 (2)

120.0

120.0

119.40 (19)

120.3

120.3

$122.7(5)$

$120.2(5)$

$117.1(6)$

$126.6(5)$

$129.1(5)$

$104.3(5)$

$109.1(7)$

109.9

109.9

109.9

109.9

108.3

106.1 (6)

110.5

110.5

110.5

110.5

108.7

$95.5(5)$

112.7

112.7

112.7

112.7

110.1

109.5

109.5

109.5

109.5

109.5

109.5

124.8 (9)

119.3 (10)

115.9 (10)

120.0 (9)

$133.8(10)$

105.1 (9)

107.5 (11)

110.2 


\begin{tabular}{|c|c|c|c|}
\hline $\mathrm{C} 10-\mathrm{C} 9-\mathrm{C} 8$ & $121.6(2)$ & $\mathrm{C} 1 \mathrm{SA}-\mathrm{C} 2 \mathrm{SA}-\mathrm{H} 2 \mathrm{~S} 3$ & 110.2 \\
\hline $\mathrm{C} 10-\mathrm{C} 9-\mathrm{H} 9 \mathrm{~A}$ & 119.2 & $\mathrm{C} 3 \mathrm{SA}-\mathrm{C} 2 \mathrm{SA}-\mathrm{H} 2 \mathrm{~S} 4$ & 110.2 \\
\hline $\mathrm{C} 8-\mathrm{C} 9-\mathrm{H} 9 \mathrm{~A}$ & 119.2 & $\mathrm{C} 1 \mathrm{SA}-\mathrm{C} 2 \mathrm{SA}-\mathrm{H} 2 \mathrm{~S} 4$ & 110.2 \\
\hline $\mathrm{C} 9-\mathrm{C} 10-\mathrm{C} 5$ & $120.7(2)$ & $\mathrm{H} 2 \mathrm{~S} 3-\mathrm{C} 2 \mathrm{SA}-\mathrm{H} 2 \mathrm{~S} 4$ & 108.5 \\
\hline $\mathrm{C} 9-\mathrm{C} 10-\mathrm{H} 10 \mathrm{~A}$ & 119.6 & $\mathrm{C} 2 \mathrm{SA}-\mathrm{C} 3 \mathrm{SA}-\mathrm{C} 4 \mathrm{SA}$ & $109.3(10)$ \\
\hline $\mathrm{C} 5-\mathrm{C} 10-\mathrm{H} 10 \mathrm{~A}$ & 119.6 & $\mathrm{C} 2 \mathrm{SA}-\mathrm{C} 3 \mathrm{SA}-\mathrm{H} 3 \mathrm{~S} 3$ & 109.8 \\
\hline $\mathrm{C} 8-\mathrm{C} 11-\mathrm{H} 11 \mathrm{~A}$ & 109.5 & $\mathrm{C} 4 \mathrm{SA}-\mathrm{C} 3 \mathrm{SA}-\mathrm{H} 3 \mathrm{~S} 3$ & 109.8 \\
\hline $\mathrm{C} 8-\mathrm{C} 11-\mathrm{H} 11 \mathrm{~B}$ & 109.5 & $\mathrm{C} 2 \mathrm{SA}-\mathrm{C} 3 \mathrm{SA}-\mathrm{H} 3 \mathrm{~S} 4$ & 109.8 \\
\hline $\mathrm{H} 11 \mathrm{~A}-\mathrm{C} 11-\mathrm{H} 11 \mathrm{~B}$ & 109.5 & $\mathrm{C} 4 \mathrm{SA}-\mathrm{C} 3 \mathrm{SA}-\mathrm{H} 3 \mathrm{~S} 4$ & 109.8 \\
\hline $\mathrm{C} 8-\mathrm{C} 11-\mathrm{H} 11 \mathrm{C}$ & 109.5 & $\mathrm{H} 3 \mathrm{~S} 3-\mathrm{C} 3 \mathrm{SA}-\mathrm{H} 3 \mathrm{~S} 4$ & 108.3 \\
\hline $\mathrm{H} 11 \mathrm{~A}-\mathrm{C} 11-\mathrm{H} 11 \mathrm{C}$ & 109.5 & $\mathrm{~N} 1 \mathrm{SA}-\mathrm{C} 4 \mathrm{SA}-\mathrm{C} 3 \mathrm{SA}$ & $92.5(8)$ \\
\hline $\mathrm{H} 11 \mathrm{~B}-\mathrm{C} 11-\mathrm{H} 11 \mathrm{C}$ & 109.5 & $\mathrm{~N} 1 \mathrm{SA}-\mathrm{C} 4 \mathrm{SA}-\mathrm{H} 4 \mathrm{~S} 3$ & 113.2 \\
\hline $\mathrm{C} 17-\mathrm{C} 12-\mathrm{C} 13$ & $120.86(19)$ & $\mathrm{C} 3 \mathrm{SA}-\mathrm{C} 4 \mathrm{SA}-\mathrm{H} 4 \mathrm{~S} 3$ & 113.2 \\
\hline $\mathrm{C} 17-\mathrm{C} 12-\mathrm{N} 2$ & $120.66(18)$ & $\mathrm{N} 1 \mathrm{SA}-\mathrm{C} 4 \mathrm{SA}-\mathrm{H} 4 \mathrm{~S} 4$ & 113.2 \\
\hline $\mathrm{C} 13-\mathrm{C} 12-\mathrm{N} 2$ & $118.40(19)$ & $\mathrm{C} 3 \mathrm{SA}-\mathrm{C} 4 \mathrm{SA}-\mathrm{H} 4 \mathrm{~S} 4$ & 113.2 \\
\hline $\mathrm{C} 14-\mathrm{C} 13-\mathrm{C} 12$ & $119.7(2)$ & $\mathrm{H} 4 \mathrm{~S} 3-\mathrm{C} 4 \mathrm{SA}-\mathrm{H} 4 \mathrm{~S} 4$ & 110.6 \\
\hline $\mathrm{C} 14-\mathrm{C} 13-\mathrm{H} 13 \mathrm{~A}$ & 120.1 & $\mathrm{~N} 1 \mathrm{SA}-\mathrm{C} 5 \mathrm{SA}-\mathrm{H} 5 \mathrm{~S} 4$ & 109.5 \\
\hline $\mathrm{C} 12-\mathrm{C} 13-\mathrm{H} 13 \mathrm{~A}$ & 120.1 & $\mathrm{~N} 1 \mathrm{SA}-\mathrm{C} 5 \mathrm{SA}-\mathrm{H} 5 \mathrm{~S} 5$ & 109.5 \\
\hline $\mathrm{C} 13-\mathrm{C} 14-\mathrm{C} 15$ & $119.7(2)$ & $\mathrm{H} 5 \mathrm{~S} 4-\mathrm{C} 5 \mathrm{SA}-\mathrm{H} 5 \mathrm{~S} 5$ & 109.5 \\
\hline $\mathrm{C} 13-\mathrm{C} 14-\mathrm{H} 14 \mathrm{~A}$ & 120.1 & $\mathrm{~N} 1 \mathrm{SA}-\mathrm{C} 5 \mathrm{SA}-\mathrm{H} 5 \mathrm{~S} 6$ & 109.5 \\
\hline $\mathrm{C} 15-\mathrm{C} 14-\mathrm{H} 14 \mathrm{~A}$ & 120.1 & $\mathrm{H} 5 \mathrm{~S} 4-\mathrm{C} 5 \mathrm{SA}-\mathrm{H} 5 \mathrm{~S} 6$ & 109.5 \\
\hline $\mathrm{C} 16-\mathrm{C} 15-\mathrm{C} 14$ & $120.31(19)$ & $\mathrm{H} 5 \mathrm{~S} 5-\mathrm{C} 5 \mathrm{SA}-\mathrm{H} 5 \mathrm{~S} 6$ & 109.5 \\
\hline $\mathrm{C} 1-\mathrm{N} 1-\mathrm{N} 2-\mathrm{C} 3$ & $0.4(2)$ & $\mathrm{C} 3-\mathrm{N} 2-\mathrm{C} 12-\mathrm{C} 17$ & $-52.0(3)$ \\
\hline $\mathrm{C} 1-\mathrm{N} 1-\mathrm{N} 2-\mathrm{C} 12$ & $-177.00(19)$ & $\mathrm{N} 1-\mathrm{N} 2-\mathrm{C} 12-\mathrm{C} 13$ & $-52.1(3)$ \\
\hline $\mathrm{N} 2-\mathrm{N} 1-\mathrm{C} 1-\mathrm{C} 2$ & $-0.2(3)$ & $\mathrm{C} 3-\mathrm{N} 2-\mathrm{C} 12-\mathrm{C} 13$ & $131.1(2)$ \\
\hline $\mathrm{N} 2-\mathrm{N} 1-\mathrm{C} 1-\mathrm{C} 4$ & $-179.1(2)$ & $\mathrm{C} 17-\mathrm{C} 12-\mathrm{C} 13-\mathrm{C} 14$ & $-0.5(4)$ \\
\hline $\mathrm{N} 1-\mathrm{C} 1-\mathrm{C} 2-\mathrm{C} 3$ & $-0.1(3)$ & $\mathrm{N} 2-\mathrm{C} 12-\mathrm{C} 13-\mathrm{C} 14$ & $176.4(2)$ \\
\hline $\mathrm{C} 4-\mathrm{C} 1-\mathrm{C} 2-\mathrm{C} 3$ & $178.7(3)$ & $\mathrm{C} 12-\mathrm{C} 13-\mathrm{C} 14-\mathrm{C} 15$ & $-0.1(4)$ \\
\hline $\mathrm{N} 1-\mathrm{N} 2-\mathrm{C} 3-\mathrm{C} 2$ & $-0.5(2)$ & $\mathrm{C} 13-\mathrm{C} 14-\mathrm{C} 15-\mathrm{C} 16$ & $0.9(4)$ \\
\hline $\mathrm{C} 12-\mathrm{N} 2-\mathrm{C} 3-\mathrm{C} 2$ & $176.5(2)$ & $\mathrm{C} 13-\mathrm{C} 14-\mathrm{C} 15-\mathrm{S} 1$ & $-179.5(2)$ \\
\hline $\mathrm{N} 1-\mathrm{N} 2-\mathrm{C} 3-\mathrm{C} 5$ & $176.31(19)$ & $\mathrm{O} 1-\mathrm{S} 1-\mathrm{C} 15-\mathrm{C} 16$ & $-160.02(19)$ \\
\hline $\mathrm{C} 12-\mathrm{N} 2-\mathrm{C} 3-\mathrm{C} 5$ & $-6.7(3)$ & $\mathrm{O} 2-\mathrm{S} 1-\mathrm{C} 15-\mathrm{C} 16$ & $-30.2(2)$ \\
\hline $\mathrm{C} 1-\mathrm{C} 2-\mathrm{C} 3-\mathrm{N} 2$ & $0.4(3)$ & $\mathrm{N} 3-\mathrm{S} 1-\mathrm{C} 15-\mathrm{C} 16$ & $84.8(2)$ \\
\hline $\mathrm{C} 1-\mathrm{C} 2-\mathrm{C} 3-\mathrm{C} 5$ & $-176.2(2)$ & $\mathrm{O} 1-\mathrm{S} 1-\mathrm{C} 15-\mathrm{C} 14$ & $20.4(2)$ \\
\hline $\mathrm{N} 1-\mathrm{C} 1-\mathrm{C} 4-\mathrm{F} 2$ & $74.9(11)$ & $\mathrm{O} 2-\mathrm{S} 1-\mathrm{C} 15-\mathrm{C} 14$ & $150.2(2)$ \\
\hline $\mathrm{C} 2-\mathrm{C} 1-\mathrm{C} 4-\mathrm{F} 2$ & $-103.9(11)$ & $\mathrm{N} 3-\mathrm{S} 1-\mathrm{C} 15-\mathrm{C} 14$ & $-94.8(2)$ \\
\hline $\mathrm{N} 1-\mathrm{C} 1-\mathrm{C} 4-\mathrm{F} 3$ & $-48.7(7)$ & $\mathrm{C} 14-\mathrm{C} 15-\mathrm{C} 16-\mathrm{C} 17$ & $-1.0(4)$ \\
\hline $\mathrm{C} 2-\mathrm{C} 1-\mathrm{C} 4-\mathrm{F} 3$ & $132.5(6)$ & $\mathrm{S} 1-\mathrm{C} 15-\mathrm{C} 16-\mathrm{C} 17$ & $179.36(18)$ \\
\hline $\mathrm{N} 1-\mathrm{C} 1-\mathrm{C} 4-\mathrm{F} 2 \mathrm{~A}$ & $133.1(5)$ & $\mathrm{C} 13-\mathrm{C} 12-\mathrm{C} 17-\mathrm{C} 16$ & $0.4(3)$ \\
\hline $\mathrm{C} 2-\mathrm{C} 1-\mathrm{C} 4-\mathrm{F} 2 \mathrm{~A}$ & $-45.7(7)$ & $\mathrm{N} 2-\mathrm{C} 12-\mathrm{C} 17-\mathrm{C} 16$ & $-176.5(2)$ \\
\hline $\mathrm{N} 1-\mathrm{C} 1-\mathrm{C} 4-\mathrm{F} 1 \mathrm{~A}$ & $-105.2(9)$ & $\mathrm{C} 15-\mathrm{C} 16-\mathrm{C} 17-\mathrm{C} 12$ & $0.4(3)$ \\
\hline $\mathrm{C} 2-\mathrm{C} 1-\mathrm{C} 4-\mathrm{F} 1 \mathrm{~A}$ & $76.1(10)$ & $\mathrm{C} 4 \mathrm{~S}-\mathrm{N} 1 \mathrm{~S}-\mathrm{C} 1 \mathrm{~S}-\mathrm{O} 1 \mathrm{~S}$ & $179.7(7)$ \\
\hline $\mathrm{N} 1-\mathrm{C} 1-\mathrm{C} 4-\mathrm{F} 1$ & $-162.2(10)$ & $\mathrm{C} 5 \mathrm{~S}-\mathrm{N} 1 \mathrm{~S}-\mathrm{C} 1 \mathrm{~S}-\mathrm{O} 1 \mathrm{~S}$ & $-0.4(11)$ \\
\hline $\mathrm{C} 2-\mathrm{C} 1-\mathrm{C} 4-\mathrm{F} 1$ & $19.0(11)$ & $\mathrm{C} 4 \mathrm{~S}-\mathrm{N} 1 \mathrm{~S}-\mathrm{C} 1 \mathrm{~S}-\mathrm{C} 2 \mathrm{~S}$ & $1.3(9)$ \\
\hline $\mathrm{N} 1-\mathrm{C} 1-\mathrm{C} 4-\mathrm{F} 3 \mathrm{~A}$ & $15.1(9)$ & $\mathrm{C} 5 \mathrm{~S}-\mathrm{N} 1 \mathrm{~S}-\mathrm{C} 1 \mathrm{~S}-\mathrm{C} 2 \mathrm{~S}$ & $-178.9(6)$ \\
\hline $\mathrm{C} 2-\mathrm{C} 1-\mathrm{C} 4-\mathrm{F} 3 \mathrm{~A}$ & $-163.6(9)$ & $\mathrm{O} 1 \mathrm{~S}-\mathrm{C} 1 \mathrm{~S}-\mathrm{C} 2 \mathrm{~S}-\mathrm{C} 3 \mathrm{~S}$ & $169.9(9)$ \\
\hline $\mathrm{N} 2-\mathrm{C} 3-\mathrm{C} 5-\mathrm{C} 6$ & $-34.0(3)$ & $\mathrm{N} 1 \mathrm{~S}-\mathrm{C} 1 \mathrm{~S}-\mathrm{C} 2 \mathrm{~S}-\mathrm{C} 3 \mathrm{~S}$ & $-11.7(10)$ \\
\hline
\end{tabular}




$\begin{array}{llll}\mathrm{C} 2-\mathrm{C} 3-\mathrm{C} 5-\mathrm{C} 6 & 142.0(2) & \mathrm{C} 1 \mathrm{~S}-\mathrm{C} 2 \mathrm{~S}-\mathrm{C} 3 \mathrm{~S}-\mathrm{C} 4 \mathrm{~S} & 15.9(10) \\ \mathrm{N} 2-\mathrm{C} 3-\mathrm{C} 5-\mathrm{C} 10 & 149.0(2) & \mathrm{C} 1 \mathrm{~S}-\mathrm{N} 1 \mathrm{~S}-\mathrm{C} 4 \mathrm{~S}-\mathrm{C} 3 \mathrm{~S} & 7.5(9) \\ \mathrm{C} 2-\mathrm{C} 3-\mathrm{C} 5-\mathrm{C} 10 & -35.0(3) & \mathrm{C} 5 \mathrm{~S}-\mathrm{N} 1 \mathrm{~S}-\mathrm{C} 4 \mathrm{~S}-\mathrm{C} 3 \mathrm{~S} & -172.3(6) \\ \mathrm{C} 10-\mathrm{C} 5-\mathrm{C} 6-\mathrm{C} 7 & -0.5(3) & \mathrm{C} 2 \mathrm{~S}-\mathrm{C} 3 \mathrm{~S}-\mathrm{C} 4 \mathrm{~S}-\mathrm{N} 1 \mathrm{~S} & -13.7(9) \\ \mathrm{C} 3-\mathrm{C} 5-\mathrm{C} 6-\mathrm{C} 7 & -177.62(19) & \mathrm{C} 4 \mathrm{SA}-\mathrm{N} 1 \mathrm{SA}-\mathrm{C} 1 \mathrm{SA}-\mathrm{O} 1 \mathrm{SA} & -169.5(15) \\ \mathrm{C} 5-\mathrm{C} 6-\mathrm{C} 7-\mathrm{C} 8 & 0.5(3) & \mathrm{C} 5 \mathrm{SA}-\mathrm{N} 1 \mathrm{SA}-\mathrm{C} 1 \mathrm{SA}-\mathrm{O} 1 \mathrm{SA} & 10(3) \\ \mathrm{C} 6-\mathrm{C} 7-\mathrm{C} 8-\mathrm{C} 9 & 0.2(3) & \mathrm{C} 4 \mathrm{SA}-\mathrm{N} 1 \mathrm{SA}-\mathrm{C} 1 \mathrm{SA}-\mathrm{C} 2 \mathrm{SA} & 1(2) \\ \mathrm{C} 6-\mathrm{C} 7-\mathrm{C} 8-\mathrm{C} 11 & -179.7(3) & \mathrm{C} 5 \mathrm{SA}-\mathrm{N} 1 \mathrm{SA}-\mathrm{C} 1 \mathrm{SA}-\mathrm{C} 2 \mathrm{SA} & -179.9(16) \\ \mathrm{C} 7-\mathrm{C} 8-\mathrm{C} 9-\mathrm{C} 10 & -0.8(4) & \mathrm{O} 1 \mathrm{SA}-\mathrm{C} 1 \mathrm{SA}-\mathrm{C} 2 \mathrm{SA}-\mathrm{C} 3 \mathrm{SA} & 174(2) \\ \mathrm{C} 11-\mathrm{C} 8-\mathrm{C} 9-\mathrm{C} 10 & 179.1(3) & \mathrm{N} 1 \mathrm{SA}-\mathrm{C} 1 \mathrm{SA}-\mathrm{C} 2 \mathrm{SA}-\mathrm{C} 3 \mathrm{SA} & 6(2) \\ \mathrm{C} 8-\mathrm{C} 9-\mathrm{C} 10-\mathrm{C} 5 & 0.7(4) & \mathrm{C} 1 \mathrm{SA}-\mathrm{C} 2 \mathrm{SA}-\mathrm{C} 3 \mathrm{SA}-\mathrm{C} 4 \mathrm{SA} & -9(2) \\ \mathrm{C} 6-\mathrm{C} 5-\mathrm{C} 10-\mathrm{C} 9 & -0.1(3) & \mathrm{C} 1 \mathrm{SA}-\mathrm{N} 1 \mathrm{SA}-\mathrm{C} 4 \mathrm{SA}-\mathrm{C} 3 \mathrm{SA} & -5(2) \\ \mathrm{C} 3-\mathrm{C} 5-\mathrm{C} 10-\mathrm{C} 9 & 177.2(2) & \mathrm{C} 5 \mathrm{SA}-\mathrm{N} 1 \mathrm{SA}-\mathrm{C} 4 \mathrm{SA}-\mathrm{C} 3 \mathrm{SA} & 175.2(16) \\ \mathrm{N} 1-\mathrm{N} 2-\mathrm{C} 12-\mathrm{C} 17 & 124.9(2) & \mathrm{C} 2 \mathrm{SA}-\mathrm{C} 3 \mathrm{SA}-\mathrm{C} 4 \mathrm{SA}-\mathrm{N} 1 \mathrm{SA} & 8(2) \\ \end{array}$

4-[5-(4-Methylphenyl)-3-(trifluoromethyl)pyrazol-1-yl]benzenesulfonamide tetramethylurea monosolvate (4)

Crystal data

$\mathrm{C}_{17} \mathrm{H}_{14} \mathrm{~F}_{3} \mathrm{~N}_{3} \mathrm{O}_{2} \mathrm{~S} \cdot \mathrm{C}_{5} \mathrm{H}_{12} \mathrm{~N}_{2} \mathrm{O}$

$M_{r}=497.54$

Monoclinic, $P 2_{1} / c$

$a=12.4050(3) \AA$

$b=8.9351(2) \AA$

$c=22.5727(6) \AA$

$\beta=98.6702(13)^{\circ}$

$V=2473.36(11) \AA^{3}$

$Z=4$

Data collection

Bruker D8-QUEST PHOTON-100

diffractometer

Radiation source: Incoatec $\mathrm{I} \mu \mathrm{S} \mathrm{Cu}$ microsource

$\omega$ and $\varphi$-scans

Absorption correction: multi-scan

(SADABS; Bruker, 2016)

$T_{\min }=0.657, T_{\max }=0.753$

27591 measured reflections

\section{Refinement}

Refinement on $F^{2}$

Least-squares matrix: full

$R\left[F^{2}>2 \sigma\left(F^{2}\right)\right]=0.046$

$w R\left(F^{2}\right)=0.142$

$S=1.03$

4397 reflections

349 parameters

15 restraints

Primary atom site location: dual
$F(000)=1040$

$D_{\mathrm{x}}=1.336 \mathrm{Mg} \mathrm{m}^{-3}$

$\mathrm{Cu} K \alpha$ radiation, $\lambda=1.54178 \AA$

Cell parameters from 9948 reflections

$\theta=3.6-66.8^{\circ}$

$\mu=1.66 \mathrm{~mm}^{-1}$

$T=298 \mathrm{~K}$

Block, colourless

$0.16 \times 0.14 \times 0.14 \mathrm{~mm}$

4397 independent reflections

3339 reflections with $I>2 \sigma(I)$

$R_{\text {int }}=0.034$

$\theta_{\max }=66.9^{\circ}, \theta_{\min }=3.6^{\circ}$

$h=-14 \rightarrow 14$

$k=-9 \rightarrow 10$

$l=-26 \rightarrow 26$

Secondary atom site location: difference Fourier map

Hydrogen site location: mixed

$\mathrm{H}$ atoms treated by a mixture of independent and constrained refinement

$w=1 /\left[\sigma^{2}\left(F_{\mathrm{o}}^{2}\right)+(0.0714 P)^{2}+0.619 P\right]$

where $P=\left(F_{\mathrm{o}}^{2}+2 F_{\mathrm{c}}^{2}\right) / 3$

$(\Delta / \sigma)_{\max }<0.001$

$\Delta \rho_{\max }=0.42 \mathrm{e} \AA^{-3}$

$\Delta \rho_{\min }=-0.20$ e $\AA^{-3}$ 


\section{Special details}

Geometry. All esds (except the esd in the dihedral angle between two 1.s. planes) are estimated using the full covariance matrix. The cell esds are taken into account individually in the estimation of esds in distances, angles and torsion angles; correlations between esds in cell parameters are only used when they are defined by crystal symmetry. An approximate (isotropic) treatment of cell esds is used for estimating esds involving l.s. planes.

Fractional atomic coordinates and isotropic or equivalent isotropic displacement parameters $\left(\AA^{2}\right)$

\begin{tabular}{|c|c|c|c|c|c|}
\hline & $x$ & $y$ & $z$ & $U_{\text {iso }} * / U_{\text {eq }}$ & Occ. $(<1)$ \\
\hline S1 & $0.42251(5)$ & $0.73033(8)$ & $0.57691(3)$ & $0.0805(2)$ & \\
\hline $\mathrm{O} 1$ & $0.39736(16)$ & $0.6847(3)$ & $0.63369(8)$ & $0.1108(7)$ & \\
\hline $\mathrm{O} 2$ & $0.37201(15)$ & $0.8603(2)$ & $0.54817(10)$ & $0.1070(6)$ & \\
\hline N1 & $0.94939(17)$ & $0.8747(2)$ & $0.65960(9)$ & $0.0771(5)$ & \\
\hline $\mathrm{N} 2$ & $0.90320(14)$ & $0.8185(2)$ & $0.60585(8)$ & $0.0655(5)$ & \\
\hline N3 & $0.39387(16)$ & $0.5931(3)$ & $0.53183(9)$ & $0.0769(5)$ & \\
\hline $\mathrm{H} 1 \mathrm{~N}$ & $0.408(2)$ & $0.620(2)$ & $0.4968(7)$ & $0.092(8)^{*}$ & \\
\hline $\mathrm{H} 2 \mathrm{~N}$ & $0.422(2)$ & $0.5081(18)$ & $0.5463(10)$ & $0.091(9)^{*}$ & \\
\hline $\mathrm{C} 1$ & $1.0536(2)$ & $0.8897(3)$ & $0.65326(12)$ & $0.0769(6)$ & \\
\hline $\mathrm{C} 2$ & $1.07521(19)$ & $0.8457(2)$ & $0.59772(11)$ & $0.0736(6)$ & \\
\hline $\mathrm{H} 2 \mathrm{~A}$ & 1.142114 & 0.847511 & 0.583867 & $0.088^{*}$ & \\
\hline $\mathrm{C} 3$ & $0.97680(17)$ & $0.7985(2)$ & $0.56714(10)$ & $0.0623(5)$ & \\
\hline $\mathrm{C} 4$ & $1.1316(3)$ & $0.9460(4)$ & $0.70503(15)$ & $0.1027(9)$ & \\
\hline F1 & $1.1353(12)$ & $1.0922(5)$ & $0.7124(5)$ & $0.157(4)$ & $0.541(17)$ \\
\hline $\mathrm{F} 2$ & $1.1137(7)$ & $0.8917(13)$ & $0.7567(3)$ & $0.129(3)$ & $0.541(17)$ \\
\hline F3 & $1.2325(4)$ & $0.9085(16)$ & $0.7014(3)$ & $0.139(3)$ & $0.541(17)$ \\
\hline F1A & $1.0843(7)$ & $1.0587(14)$ & $0.7295(4)$ & $0.151(4)$ & $0.459(17)$ \\
\hline $\mathrm{F} 2 \mathrm{~A}$ & $1.1571(16)$ & $0.8507(12)$ & $0.7464(6)$ & $0.211(8)$ & $0.459(17)$ \\
\hline F3A & $1.2167(10)$ & $0.999(2)$ & $0.6878(6)$ & $0.195(6)$ & $0.459(17)$ \\
\hline $\mathrm{C} 5$ & $0.95104(16)$ & $0.7317(2)$ & $0.50748(10)$ & $0.0586(5)$ & \\
\hline C6 & $0.88179(16)$ & $0.6091(2)$ & $0.49569(10)$ & $0.0599(5)$ & \\
\hline H6A & 0.847648 & 0.569658 & 0.526178 & $0.072^{*}$ & \\
\hline $\mathrm{C} 7$ & $0.86323(16)$ & $0.5455(2)$ & $0.43971(10)$ & $0.0645(5)$ & \\
\hline H7A & 0.816579 & 0.463763 & 0.433008 & $0.077^{*}$ & \\
\hline $\mathrm{C} 8$ & $0.91224(18)$ & $0.6002(3)$ & $0.39311(10)$ & $0.0698(6)$ & \\
\hline C9 & $0.9805(2)$ & $0.7221(3)$ & $0.40481(11)$ & $0.0769(6)$ & \\
\hline H9A & 1.013960 & 0.761633 & 0.374068 & $0.092^{*}$ & \\
\hline $\mathrm{C} 10$ & $1.00050(18)$ & $0.7867(3)$ & $0.46071(11)$ & $0.0719(6)$ & \\
\hline H10A & 1.047615 & 0.867941 & 0.467234 & $0.086^{*}$ & \\
\hline $\mathrm{C} 11$ & $0.8907(3)$ & $0.5301(4)$ & $0.33170(12)$ & $0.0992(9)$ & \\
\hline H11A & 0.880615 & 0.607387 & 0.301832 & $0.149^{*}$ & \\
\hline H11B & 0.826048 & 0.469671 & 0.328501 & $0.149^{*}$ & \\
\hline H11C & 0.951543 & 0.468655 & 0.325674 & $0.149^{*}$ & \\
\hline $\mathrm{C} 12$ & $0.78759(17)$ & $0.7950(2)$ & $0.59725(10)$ & $0.0614(5)$ & \\
\hline $\mathrm{C} 13$ & $0.7437(2)$ & $0.7113(3)$ & $0.63911(10)$ & $0.0731(6)$ & \\
\hline H13A & 0.788827 & 0.668236 & 0.671245 & $0.088^{*}$ & \\
\hline $\mathrm{C} 14$ & $0.6324(2)$ & $0.6922(3)$ & $0.63282(11)$ & $0.0742(6)$ & \\
\hline H14A & 0.602035 & 0.635395 & 0.660624 & $0.089^{*}$ & \\
\hline $\mathrm{C} 15$ & $0.56598(18)$ & $0.7575(2)$ & $0.58518(10)$ & $0.0663(5)$ & \\
\hline
\end{tabular}




$\begin{array}{lllll}\text { C16 } & 0.61042(18) & 0.8404(3) & 0.54350(10) & 0.0677(6) \\ \text { H16A } & 0.565409 & 0.883539 & 0.511356 & 0.081^{*} \\ \text { C17 } & 0.72232(18) & 0.8594(2) & 0.54957(10) & 0.0645(5) \\ \text { H17A } & 0.752950 & 0.915223 & 0.521583 & 0.077^{*} \\ \text { O1S } & 0.47057(13) & 0.6652(2) & 0.42142(8) & 0.0869(5) \\ \text { N1S } & 0.53109(19) & 0.7639(3) & 0.34089(9) & 0.0902(7) \\ \text { N2S } & 0.34946(17) & 0.7769(3) & 0.35146(9) & 0.0835(6) \\ \text { C1S } & 0.45072(18) & 0.7314(3) & 0.37302(10) & 0.0662(5) \\ \text { C2S } & 0.6425(3) & 0.7372(5) & 0.36927(19) & 0.1527(18) \\ \text { H1S } & 0.648920 & 0.759700 & 0.411246 & 0.229^{*} \\ \text { H2S } & 0.691099 & 0.800189 & 0.351168 & 0.229^{*} \\ \text { H3S } & 0.660995 & 0.634216 & 0.364152 & 0.229^{*} \\ \text { C3S } & 0.5154(4) & 0.7437(6) & 0.27663(17) & 0.1638(19) \\ \text { H4S } & 0.444587 & 0.701588 & 0.263666 & 0.246^{*} \\ \text { H5S } & 0.570328 & 0.677299 & 0.266065 & 0.246^{*} \\ \text { H6S } & 0.520929 & 0.838723 & 0.257482 & 0.246^{*} \\ \text { C4S } & 0.2627(2) & 0.7334(4) & 0.38412(15) & 0.1158(11) \\ \text { H7S } & 0.275181 & 0.633146 & 0.398838 & 0.174^{*} \\ \text { H8S } & 0.194159 & 0.737855 & 0.357951 & 0.174^{*} \\ \text { H9S } & 0.261118 & 0.800385 & 0.417233 & 0.174^{*} \\ \text { C5S } & 0.3256(3) & 0.9007(4) & 0.31098(17) & 0.1307(13) \\ \text { H10S } & 0.389073 & 0.962898 & 0.312511 & 0.196^{*} \\ \text { H11S } & 0.266742 & 0.958313 & 0.322455 & 0.196^{*} \\ \text { H12S } & 0.305013 & 0.863733 & 0.270950 & 0.196^{*} \\ & & & & \end{array}$

Atomic displacement parameters $\left(\AA^{2}\right)$

\begin{tabular}{lllllll}
\hline & $U^{11}$ & $U^{22}$ & $U^{33}$ & $U^{12}$ & $U^{13}$ & $U^{23}$ \\
\hline S1 & $0.0617(4)$ & $0.1053(5)$ & $0.0791(4)$ & $0.0079(3)$ & $0.0257(3)$ & $0.0038(3)$ \\
O1 & $0.0878(13)$ & $0.174(2)$ & $0.0789(12)$ & $-0.0065(13)$ & $0.0414(10)$ & $-0.0020(12)$ \\
O2 & $0.0733(11)$ & $0.1056(14)$ & $0.1441(17)$ & $0.0316(10)$ & $0.0231(11)$ & $0.0122(12)$ \\
N1 & $0.0803(14)$ & $0.0787(13)$ & $0.0671(12)$ & $-0.0039(10)$ & $-0.0060(10)$ & $-0.0047(9)$ \\
N2 & $0.0632(11)$ & $0.0658(10)$ & $0.0646(11)$ & $0.0004(8)$ & $-0.0002(9)$ & $-0.0018(8)$ \\
N3 & $0.0641(12)$ & $0.0997(16)$ & $0.0694(13)$ & $-0.0055(10)$ & $0.0177(10)$ & $0.0149(11)$ \\
C1 & $0.0785(16)$ & $0.0685(14)$ & $0.0775(16)$ & $-0.0091(11)$ & $-0.0084(12)$ & $0.0016(11)$ \\
C2 & $0.0640(13)$ & $0.0687(13)$ & $0.0842(16)$ & $-0.0067(10)$ & $-0.0016(12)$ & $0.0056(12)$ \\
C3 & $0.0584(12)$ & $0.0541(11)$ & $0.0718(13)$ & $-0.0011(9)$ & $0.0011(10)$ & $0.0056(9)$ \\
C4 & $0.105(3)$ & $0.102(2)$ & $0.090(2)$ & $-0.017(2)$ & $-0.0200(18)$ & $-0.0046(19)$ \\
F1 & $0.203(9)$ & $0.081(3)$ & $0.151(6)$ & $-0.031(4)$ & $-0.085(6)$ & $-0.011(3)$ \\
F2 & $0.107(4)$ & $0.204(8)$ & $0.067(3)$ & $-0.033(4)$ & $-0.017(3)$ & $0.026(3)$ \\
F3 & $0.072(3)$ & $0.216(9)$ & $0.120(5)$ & $-0.011(4)$ & $-0.017(2)$ & $-0.032(5)$ \\
F1A & $0.161(6)$ & $0.174(8)$ & $0.105(5)$ & $-0.063(5)$ & $-0.022(4)$ & $-0.045(5)$ \\
F2A & $0.241(16)$ & $0.148(6)$ & $0.191(12)$ & $-0.029(8)$ & $-0.137(10)$ & $0.055(7)$ \\
F3A & $0.153(9)$ & $0.234(13)$ & $0.181(7)$ & $-0.127(9)$ & $-0.026(7)$ & $-0.018(9)$ \\
C5 & $0.0477(10)$ & $0.0579(11)$ & $0.0687(13)$ & $0.0040(8)$ & $0.0040(9)$ & $0.0062(9)$ \\
C6 & $0.0502(11)$ & $0.0598(11)$ & $0.0704(13)$ & $0.0029(9)$ & $0.0114(9)$ & $0.0042(10)$ \\
C7 & $0.0518(11)$ & $0.0650(12)$ & $0.0766(14)$ & $0.0043(9)$ & $0.0090(10)$ & $-0.0057(10)$ \\
C8 & $0.0587(13)$ & $0.0789(14)$ & $0.0716(14)$ & $0.0134(11)$ & $0.0096(11)$ & $-0.0012(11)$
\end{tabular}


supporting information

$\begin{array}{lllllll} & & & & & \\ \text { C9 } & 0.0662(14) & 0.0926(17) & 0.0750(16) & 0.0034(12) & 0.0201(12) & 0.0121(13) \\ \text { C10 } & 0.0594(13) & 0.0722(14) & 0.0838(16) & -0.0075(10) & 0.0099(11) & 0.0081(12) \\ \text { C11 } & 0.111(2) & 0.112(2) & 0.0752(17) & 0.0125(17) & 0.0173(15) & -0.0116(15) \\ \text { C12 } & 0.0612(12) & 0.0616(12) & 0.0603(12) & 0.0029(9) & 0.0063(10) & -0.0038(9) \\ \text { C13 } & 0.0757(15) & 0.0789(15) & 0.0624(13) & 0.0044(11) & 0.0028(11) & 0.0115(11) \\ \text { C14 } & 0.0751(15) & 0.0834(15) & 0.0661(14) & -0.0015(12) & 0.0171(11) & 0.0101(11) \\ \text { C15 } & 0.0611(12) & 0.0744(13) & 0.0651(13) & 0.0054(10) & 0.0155(10) & -0.0010(10) \\ \text { C16 } & 0.0613(13) & 0.0782(14) & 0.0637(13) & 0.0107(10) & 0.0098(10) & 0.0073(11) \\ \text { C17 } & 0.0635(13) & 0.0675(13) & 0.0629(12) & 0.0039(10) & 0.0110(10) & 0.0074(10) \\ \text { O1S } & 0.0702(10) & 0.1191(14) & 0.0737(11) & 0.0058(9) & 0.0183(8) & 0.0310(10) \\ \text { N1S } & 0.0824(14) & 0.1250(18) & 0.0674(13) & 0.0110(12) & 0.0246(11) & 0.0276(12) \\ \text { N2S } & 0.0707(13) & 0.1075(16) & 0.0687(12) & 0.0160(11) & -0.0010(10) & -0.0045(11) \\ \text { C1S } & 0.0650(13) & 0.0789(14) & 0.0540(12) & 0.0045(10) & 0.0064(10) & 0.0001(10) \\ \text { C2S } & 0.0701(19) & 0.237(5) & 0.159(3) & 0.011(2) & 0.042(2) & 0.090(3) \\ \text { C3S } & 0.201(5) & 0.215(5) & 0.088(2) & 0.045(4) & 0.061(3) & 0.020(3) \\ \text { C4S } & 0.0594(16) & 0.177(3) & 0.109(2) & 0.0090(18) & 0.0084(15) & -0.019(2) \\ \text { C5S } & 0.140(3) & 0.116(3) & 0.128(3) & 0.052(2) & -0.007(2) & 0.016(2) \\ & & & & & \end{array}$

Geometric parameters $\left(\AA,{ }^{\circ}\right)$

\begin{tabular}{llll}
\hline $\mathrm{S} 1-\mathrm{O} 1$ & $1.4236(19)$ & $\mathrm{C} 11-\mathrm{H} 11 \mathrm{~A}$ & 0.9600 \\
$\mathrm{~S} 1-\mathrm{O} 2$ & $1.428(2)$ & $\mathrm{C} 11-\mathrm{H} 11 \mathrm{~B}$ & 0.9600 \\
$\mathrm{~S} 1-\mathrm{N} 3$ & $1.599(2)$ & $\mathrm{C} 11-\mathrm{H} 11 \mathrm{C}$ & 0.9600 \\
$\mathrm{~S} 1-\mathrm{C} 15$ & $1.778(2)$ & $\mathrm{C} 12-\mathrm{C} 17$ & $1.372(3)$ \\
$\mathrm{N} 1-\mathrm{C} 1$ & $1.329(3)$ & $\mathrm{C} 12-\mathrm{C} 13$ & $1.380(3)$ \\
$\mathrm{N} 1-\mathrm{N} 2$ & $1.357(2)$ & $\mathrm{C} 13-\mathrm{C} 14$ & $1.377(3)$ \\
$\mathrm{N} 2-\mathrm{C} 3$ & $1.367(3)$ & $\mathrm{C} 13-\mathrm{H} 13 \mathrm{~A}$ & 0.9300 \\
$\mathrm{~N} 2-\mathrm{C} 12$ & $1.433(3)$ & $\mathrm{C} 14-\mathrm{C} 15$ & $1.382(3)$ \\
$\mathrm{N} 3-\mathrm{H} 1 \mathrm{~N}$ & $0.870(9)$ & $\mathrm{C} 14-\mathrm{H} 14 \mathrm{~A}$ & 0.9300 \\
$\mathrm{~N} 3-\mathrm{H} 2 \mathrm{~N}$ & $0.876(9)$ & $\mathrm{C} 15-\mathrm{C} 16$ & $1.376(3)$ \\
$\mathrm{C} 1-\mathrm{C} 2$ & $1.378(3)$ & $\mathrm{C} 16-\mathrm{C} 17$ & $1.385(3)$ \\
$\mathrm{C} 1-\mathrm{C} 4$ & $1.487(4)$ & $\mathrm{C} 16-\mathrm{H} 16 \mathrm{~A}$ & 0.9300 \\
$\mathrm{C} 2-\mathrm{C} 3$ & $1.375(3)$ & $\mathrm{C} 17-\mathrm{H} 17 \mathrm{~A}$ & 0.9300 \\
$\mathrm{C} 2-\mathrm{H} 2 \mathrm{~A}$ & 0.9300 & $\mathrm{O} 1 \mathrm{~S}-\mathrm{C} 1 \mathrm{~S}$ & $1.234(3)$ \\
$\mathrm{C} 3-\mathrm{C} 5$ & $1.464(3)$ & $\mathrm{N} 1 \mathrm{~S}-\mathrm{C} 1 \mathrm{~S}$ & $1.350(3)$ \\
$\mathrm{C} 4-\mathrm{F} 2 \mathrm{~A}$ & $1.269(7)$ & $\mathrm{N} 1 \mathrm{~S}-\mathrm{C} 3 \mathrm{~S}$ & $1.446(4)$ \\
$\mathrm{C} 4-\mathrm{F} 3 \mathrm{~A}$ & $1.271(7)$ & $\mathrm{N} 1 \mathrm{~S}-\mathrm{C} 2 \mathrm{~S}$ & $1.452(4)$ \\
$\mathrm{C} 4-\mathrm{F} 3$ & $1.310(6)$ & $\mathrm{N} 2 \mathrm{~S}-\mathrm{C} 1 \mathrm{~S}$ & $1.340(3)$ \\
$\mathrm{C} 4-\mathrm{F} 2$ & $1.312(6)$ & $\mathrm{N} 2 \mathrm{~S}-\mathrm{C} 5 \mathrm{~S}$ & $1.437(4)$ \\
$\mathrm{C} 4-\mathrm{F} 1$ & $\mathrm{~N} 2 \mathrm{~S}-\mathrm{C} 4 \mathrm{~S}$ & $1.447(4)$ \\
$\mathrm{C} 4-\mathrm{F} 1 \mathrm{~A}$ & $1.317(5)$ & $\mathrm{C} 2 \mathrm{~S}-\mathrm{H} 1 \mathrm{~S}$ & 0.9600 \\
$\mathrm{C} 5-\mathrm{C} 10$ & $1.327(7)$ & $\mathrm{C} 2 \mathrm{~S}-\mathrm{H} 2 \mathrm{~S}$ & 0.9600 \\
$\mathrm{C} 5-\mathrm{C} 6$ & $1.388(3)$ & $\mathrm{C} 2 \mathrm{~S}-\mathrm{H} 3 \mathrm{~S}$ & 0.9600 \\
$\mathrm{C} 6-\mathrm{C} 7$ & $1.393(3)$ & $\mathrm{C} 3 \mathrm{~S}-\mathrm{H} 4 \mathrm{~S}$ & 0.9600 \\
$\mathrm{C} 6-\mathrm{H} 6 \mathrm{~A}$ & $1.373(3)$ & $\mathrm{C} 3 \mathrm{~S}-\mathrm{H} 5 \mathrm{~S}$ & 0.9600 \\
$\mathrm{C} 7-\mathrm{C} 8$ & 0.9300 & $\mathrm{C} 4 \mathrm{~S}-\mathrm{H} 7 \mathrm{~S}$ & 0.9600 \\
$\mathrm{C} 7-\mathrm{H} 7 \mathrm{~S}$ & $1.381(3)$ & $\mathrm{C} 4 \mathrm{~S}-\mathrm{H} 8 \mathrm{~S}$ & 0.9600 \\
$\mathrm{C} 8-\mathrm{C} 9$ & 0.9300 & & 0.9600
\end{tabular}




\begin{tabular}{|c|c|c|c|}
\hline $\mathrm{C} 8-\mathrm{C} 11$ & $1.508(3)$ & $\mathrm{C} 4 \mathrm{~S}-\mathrm{H} 9 \mathrm{~S}$ & 0.9600 \\
\hline $\mathrm{C} 9-\mathrm{C} 10$ & $1.375(3)$ & $\mathrm{C} 5 \mathrm{~S}-\mathrm{H} 10 \mathrm{~S}$ & 0.9600 \\
\hline C9- $\mathrm{H} 9 \mathrm{~A}$ & 0.9300 & $\mathrm{C} 5 \mathrm{~S}-\mathrm{H} 11 \mathrm{~S}$ & 0.9600 \\
\hline $\mathrm{C} 10-\mathrm{H} 10 \mathrm{~A}$ & 0.9300 & $\mathrm{C} 5 \mathrm{~S}-\mathrm{H} 12 \mathrm{~S}$ & 0.9600 \\
\hline $\mathrm{O} 1-\mathrm{S} 1-\mathrm{O} 2$ & $120.07(13)$ & $\mathrm{C} 8-\mathrm{C} 11-\mathrm{H} 11 \mathrm{C}$ & 109.5 \\
\hline $\mathrm{O} 1-\mathrm{S} 1-\mathrm{N} 3$ & $107.20(13)$ & $\mathrm{H} 11 \mathrm{~A}-\mathrm{C} 11-\mathrm{H} 11 \mathrm{C}$ & 109.5 \\
\hline $\mathrm{O} 2-\mathrm{S} 1-\mathrm{N} 3$ & $107.12(13)$ & $\mathrm{H} 11 \mathrm{~B}-\mathrm{C} 11-\mathrm{H} 11 \mathrm{C}$ & 109.5 \\
\hline $\mathrm{O} 1-\mathrm{S} 1-\mathrm{C} 15$ & $107.23(12)$ & $\mathrm{C} 17-\mathrm{C} 12-\mathrm{C} 13$ & $121.2(2)$ \\
\hline $\mathrm{O} 2-\mathrm{S} 1-\mathrm{C} 15$ & $107.49(12)$ & $\mathrm{C} 17-\mathrm{C} 12-\mathrm{N} 2$ & $120.33(19)$ \\
\hline N3-S1-C15 & $107.12(10)$ & $\mathrm{C} 13-\mathrm{C} 12-\mathrm{N} 2$ & $118.46(19)$ \\
\hline $\mathrm{C} 1-\mathrm{N} 1-\mathrm{N} 2$ & $103.2(2)$ & $\mathrm{C} 14-\mathrm{C} 13-\mathrm{C} 12$ & $119.3(2)$ \\
\hline $\mathrm{N} 1-\mathrm{N} 2-\mathrm{C} 3$ & $112.76(18)$ & $\mathrm{C} 14-\mathrm{C} 13-\mathrm{H} 13 \mathrm{~A}$ & 120.3 \\
\hline $\mathrm{N} 1-\mathrm{N} 2-\mathrm{C} 12$ & $117.02(18)$ & $\mathrm{C} 12-\mathrm{C} 13-\mathrm{H} 13 \mathrm{~A}$ & 120.3 \\
\hline $\mathrm{C} 3-\mathrm{N} 2-\mathrm{C} 12$ & $130.17(18)$ & $\mathrm{C} 13-\mathrm{C} 14-\mathrm{C} 15$ & $120.0(2)$ \\
\hline $\mathrm{S} 1-\mathrm{N} 3-\mathrm{H} 1 \mathrm{~N}$ & $108.0(17)$ & $\mathrm{C} 13-\mathrm{C} 14-\mathrm{H} 14 \mathrm{~A}$ & 120.0 \\
\hline $\mathrm{S} 1-\mathrm{N} 3-\mathrm{H} 2 \mathrm{~N}$ & $113.0(18)$ & $\mathrm{C} 15-\mathrm{C} 14-\mathrm{H} 14 \mathrm{~A}$ & 120.0 \\
\hline $\mathrm{H} 1 \mathrm{~N}-\mathrm{N} 3-\mathrm{H} 2 \mathrm{~N}$ & $117.2(16)$ & $\mathrm{C} 16-\mathrm{C} 15-\mathrm{C} 14$ & $120.4(2)$ \\
\hline $\mathrm{N} 1-\mathrm{C} 1-\mathrm{C} 2$ & $113.3(2)$ & $\mathrm{C} 16-\mathrm{C} 15-\mathrm{S} 1$ & $119.96(18)$ \\
\hline $\mathrm{N} 1-\mathrm{C} 1-\mathrm{C} 4$ & $118.5(3)$ & $\mathrm{C} 14-\mathrm{C} 15-\mathrm{S} 1$ & $119.65(18)$ \\
\hline $\mathrm{C} 2-\mathrm{C} 1-\mathrm{C} 4$ & $128.2(3)$ & $\mathrm{C} 15-\mathrm{C} 16-\mathrm{C} 17$ & $119.8(2)$ \\
\hline $\mathrm{C} 3-\mathrm{C} 2-\mathrm{C} 1$ & $105.3(2)$ & $\mathrm{C} 15-\mathrm{C} 16-\mathrm{H} 16 \mathrm{~A}$ & 120.1 \\
\hline $\mathrm{C} 3-\mathrm{C} 2-\mathrm{H} 2 \mathrm{~A}$ & 127.3 & $\mathrm{C} 17-\mathrm{C} 16-\mathrm{H} 16 \mathrm{~A}$ & 120.1 \\
\hline $\mathrm{C} 1-\mathrm{C} 2-\mathrm{H} 2 \mathrm{~A}$ & 127.3 & $\mathrm{C} 12-\mathrm{C} 17-\mathrm{C} 16$ & $119.4(2)$ \\
\hline $\mathrm{N} 2-\mathrm{C} 3-\mathrm{C} 2$ & $105.5(2)$ & $\mathrm{C} 12-\mathrm{C} 17-\mathrm{H} 17 \mathrm{~A}$ & 120.3 \\
\hline $\mathrm{N} 2-\mathrm{C} 3-\mathrm{C} 5$ & $124.54(18)$ & $\mathrm{C} 16-\mathrm{C} 17-\mathrm{H} 17 \mathrm{~A}$ & 120.3 \\
\hline $\mathrm{C} 2-\mathrm{C} 3-\mathrm{C} 5$ & $129.9(2)$ & $\mathrm{C} 1 \mathrm{~S}-\mathrm{N} 1 \mathrm{~S}-\mathrm{C} 3 \mathrm{~S}$ & $120.9(3)$ \\
\hline $\mathrm{F} 2 \mathrm{~A}-\mathrm{C} 4-\mathrm{F} 3 \mathrm{~A}$ & $110.5(6)$ & $\mathrm{C} 1 \mathrm{~S}-\mathrm{N} 1 \mathrm{~S}-\mathrm{C} 2 \mathrm{~S}$ & $117.3(2)$ \\
\hline $\mathrm{F} 3-\mathrm{C} 4-\mathrm{F} 2$ & $104.6(5)$ & $\mathrm{C} 3 \mathrm{~S}-\mathrm{N} 1 \mathrm{~S}-\mathrm{C} 2 \mathrm{~S}$ & $113.4(3)$ \\
\hline $\mathrm{F} 3-\mathrm{C} 4-\mathrm{F} 1$ & $104.2(5)$ & $\mathrm{C} 1 \mathrm{~S}-\mathrm{N} 2 \mathrm{~S}-\mathrm{C} 5 \mathrm{~S}$ & $123.8(3)$ \\
\hline $\mathrm{F} 2-\mathrm{C} 4-\mathrm{F} 1$ & $105.1(6)$ & $\mathrm{C} 1 \mathrm{~S}-\mathrm{N} 2 \mathrm{~S}-\mathrm{C} 4 \mathrm{~S}$ & $117.5(2)$ \\
\hline $\mathrm{F} 2 \mathrm{~A}-\mathrm{C} 4-\mathrm{F} 1 \mathrm{~A}$ & $106.2(7)$ & $\mathrm{C} 5 \mathrm{~S}-\mathrm{N} 2 \mathrm{~S}-\mathrm{C} 4 \mathrm{~S}$ & $115.7(3)$ \\
\hline $\mathrm{F} 3 \mathrm{~A}-\mathrm{C} 4-\mathrm{F} 1 \mathrm{~A}$ & $106.5(6)$ & $\mathrm{O} 1 \mathrm{~S}-\mathrm{C} 1 \mathrm{~S}-\mathrm{N} 2 \mathrm{~S}$ & $121.2(2)$ \\
\hline $\mathrm{F} 2 \mathrm{~A}-\mathrm{C} 4-\mathrm{C} 1$ & $114.4(6)$ & $\mathrm{O} 1 \mathrm{~S}-\mathrm{C} 1 \mathrm{~S}-\mathrm{N} 1 \mathrm{~S}$ & $120.9(2)$ \\
\hline $\mathrm{F} 3 \mathrm{~A}-\mathrm{C} 4-\mathrm{C} 1$ & $111.0(6)$ & $\mathrm{N} 2 \mathrm{~S}-\mathrm{C} 1 \mathrm{~S}-\mathrm{N} 1 \mathrm{~S}$ & $117.9(2)$ \\
\hline $\mathrm{F} 3-\mathrm{C} 4-\mathrm{C} 1$ & $112.2(5)$ & $\mathrm{N} 1 \mathrm{~S}-\mathrm{C} 2 \mathrm{~S}-\mathrm{H} 1 \mathrm{~S}$ & 109.5 \\
\hline $\mathrm{F} 2-\mathrm{C} 4-\mathrm{C} 1$ & $113.4(5)$ & $\mathrm{N} 1 \mathrm{~S}-\mathrm{C} 2 \mathrm{~S}-\mathrm{H} 2 \mathrm{~S}$ & 109.5 \\
\hline $\mathrm{F} 1-\mathrm{C} 4-\mathrm{C} 1$ & $116.2(4)$ & $\mathrm{H} 1 \mathrm{~S}-\mathrm{C} 2 \mathrm{~S}-\mathrm{H} 2 \mathrm{~S}$ & 109.5 \\
\hline $\mathrm{F} 1 \mathrm{~A}-\mathrm{C} 4-\mathrm{C} 1$ & $107.9(4)$ & $\mathrm{N} 1 \mathrm{~S}-\mathrm{C} 2 \mathrm{~S}-\mathrm{H} 3 \mathrm{~S}$ & 109.5 \\
\hline $\mathrm{C} 10-\mathrm{C} 5-\mathrm{C} 6$ & $117.6(2)$ & $\mathrm{H} 1 \mathrm{~S}-\mathrm{C} 2 \mathrm{~S}-\mathrm{H} 3 \mathrm{~S}$ & 109.5 \\
\hline $\mathrm{C} 10-\mathrm{C} 5-\mathrm{C} 3$ & $119.70(19)$ & $\mathrm{H} 2 \mathrm{~S}-\mathrm{C} 2 \mathrm{~S}-\mathrm{H} 3 \mathrm{~S}$ & 109.5 \\
\hline $\mathrm{C} 6-\mathrm{C} 5-\mathrm{C} 3$ & $122.65(19)$ & $\mathrm{N} 1 \mathrm{~S}-\mathrm{C} 3 \mathrm{~S}-\mathrm{H} 4 \mathrm{~S}$ & 109.5 \\
\hline $\mathrm{C} 7-\mathrm{C} 6-\mathrm{C} 5$ & $121.0(2)$ & $\mathrm{N} 1 \mathrm{~S}-\mathrm{C} 3 \mathrm{~S}-\mathrm{H} 5 \mathrm{~S}$ & 109.5 \\
\hline $\mathrm{C} 7-\mathrm{C} 6-\mathrm{H} 6 \mathrm{~A}$ & 119.5 & $\mathrm{H} 4 \mathrm{~S}-\mathrm{C} 3 \mathrm{~S}-\mathrm{H} 5 \mathrm{~S}$ & 109.5 \\
\hline $\mathrm{C} 5-\mathrm{C} 6-\mathrm{H} 6 \mathrm{~A}$ & 119.5 & $\mathrm{~N} 1 \mathrm{~S}-\mathrm{C} 3 \mathrm{~S}-\mathrm{H} 6 \mathrm{~S}$ & 109.5 \\
\hline $\mathrm{C} 6-\mathrm{C} 7-\mathrm{C} 8$ & $121.5(2)$ & $\mathrm{H} 4 \mathrm{~S}-\mathrm{C} 3 \mathrm{~S}-\mathrm{H} 6 \mathrm{~S}$ & 109.5 \\
\hline $\mathrm{C} 6-\mathrm{C} 7-\mathrm{H} 7 \mathrm{~A}$ & 119.2 & $\mathrm{H} 5 \mathrm{~S}-\mathrm{C} 3 \mathrm{~S}-\mathrm{H} 6 \mathrm{~S}$ & 109.5 \\
\hline $\mathrm{C} 8-\mathrm{C} 7-\mathrm{H} 7 \mathrm{~A}$ & 119.2 & $\mathrm{~N} 2 \mathrm{~S}-\mathrm{C} 4 \mathrm{~S}-\mathrm{H} 7 \mathrm{~S}$ & 109.5 \\
\hline
\end{tabular}




\begin{tabular}{|c|c|c|c|}
\hline $\mathrm{C} 9-\mathrm{C} 8-\mathrm{C} 7$ & $117.5(2)$ & $\mathrm{N} 2 \mathrm{~S}-\mathrm{C} 4 \mathrm{~S}-\mathrm{H} 8 \mathrm{~S}$ & 109.5 \\
\hline $\mathrm{C} 9-\mathrm{C} 8-\mathrm{C} 11$ & $121.5(2)$ & $\mathrm{H} 7 \mathrm{~S}-\mathrm{C} 4 \mathrm{~S}-\mathrm{H} 8 \mathrm{~S}$ & 109.5 \\
\hline $\mathrm{C} 7-\mathrm{C} 8-\mathrm{C} 11$ & $121.0(2)$ & $\mathrm{N} 2 \mathrm{~S}-\mathrm{C} 4 \mathrm{~S}-\mathrm{H} 9 \mathrm{~S}$ & 109.5 \\
\hline $\mathrm{C} 10-\mathrm{C} 9-\mathrm{C} 8$ & $121.8(2)$ & $\mathrm{H} 7 \mathrm{~S}-\mathrm{C} 4 \mathrm{~S}-\mathrm{H} 9 \mathrm{~S}$ & 109.5 \\
\hline $\mathrm{C} 10-\mathrm{C} 9-\mathrm{H} 9 \mathrm{~A}$ & 119.1 & $\mathrm{H} 8 \mathrm{~S}-\mathrm{C} 4 \mathrm{~S}-\mathrm{H} 9 \mathrm{~S}$ & 109.5 \\
\hline $\mathrm{C} 8-\mathrm{C} 9-\mathrm{H} 9 \mathrm{~A}$ & 119.1 & $\mathrm{~N} 2 \mathrm{~S}-\mathrm{C} 5 \mathrm{~S}-\mathrm{H} 10 \mathrm{~S}$ & 109.5 \\
\hline $\mathrm{C} 9-\mathrm{C} 10-\mathrm{C} 5$ & $120.7(2)$ & $\mathrm{N} 2 \mathrm{~S}-\mathrm{C} 5 \mathrm{~S}-\mathrm{H} 11 \mathrm{~S}$ & 109.5 \\
\hline $\mathrm{C} 9-\mathrm{C} 10-\mathrm{H} 10 \mathrm{~A}$ & 119.7 & $\mathrm{H} 10 \mathrm{~S}-\mathrm{C} 5 \mathrm{~S}-\mathrm{H} 11 \mathrm{~S}$ & 109.5 \\
\hline $\mathrm{C} 5-\mathrm{C} 10-\mathrm{H} 10 \mathrm{~A}$ & 119.7 & $\mathrm{~N} 2 \mathrm{~S}-\mathrm{C} 5 \mathrm{~S}-\mathrm{H} 12 \mathrm{~S}$ & 109.5 \\
\hline $\mathrm{C} 8-\mathrm{C} 11-\mathrm{H} 11 \mathrm{~A}$ & 109.5 & $\mathrm{H} 10 \mathrm{~S}-\mathrm{C} 5 \mathrm{~S}-\mathrm{H} 12 \mathrm{~S}$ & 109.5 \\
\hline $\mathrm{C} 8-\mathrm{C} 11-\mathrm{H} 11 \mathrm{~B}$ & 109.5 & $\mathrm{H} 11 \mathrm{~S}-\mathrm{C} 5 \mathrm{~S}-\mathrm{H} 12 \mathrm{~S}$ & 109.5 \\
\hline $\mathrm{H} 11 \mathrm{~A}-\mathrm{C} 11-\mathrm{H} 11 \mathrm{~B}$ & 109.5 & & \\
\hline $\mathrm{C} 1-\mathrm{N} 1-\mathrm{N} 2-\mathrm{C} 3$ & $0.2(2)$ & $\mathrm{C} 7-\mathrm{C} 8-\mathrm{C} 9-\mathrm{C} 10$ & $0.6(3)$ \\
\hline $\mathrm{C} 1-\mathrm{N} 1-\mathrm{N} 2-\mathrm{C} 12$ & $-177.43(18)$ & $\mathrm{C} 11-\mathrm{C} 8-\mathrm{C} 9-\mathrm{C} 10$ & $179.9(2)$ \\
\hline $\mathrm{N} 2-\mathrm{N} 1-\mathrm{C} 1-\mathrm{C} 2$ & $0.1(3)$ & $\mathrm{C} 8-\mathrm{C} 9-\mathrm{C} 10-\mathrm{C} 5$ & $-0.8(4)$ \\
\hline $\mathrm{N} 2-\mathrm{N} 1-\mathrm{C} 1-\mathrm{C} 4$ & $-178.4(2)$ & $\mathrm{C} 6-\mathrm{C} 5-\mathrm{C} 10-\mathrm{C} 9$ & $0.5(3)$ \\
\hline $\mathrm{N} 1-\mathrm{C} 1-\mathrm{C} 2-\mathrm{C} 3$ & $-0.4(3)$ & $\mathrm{C} 3-\mathrm{C} 5-\mathrm{C} 10-\mathrm{C} 9$ & $177.6(2)$ \\
\hline $\mathrm{C} 4-\mathrm{C} 1-\mathrm{C} 2-\mathrm{C} 3$ & $177.9(3)$ & $\mathrm{N} 1-\mathrm{N} 2-\mathrm{C} 12-\mathrm{C} 17$ & $124.6(2)$ \\
\hline $\mathrm{N} 1-\mathrm{N} 2-\mathrm{C} 3-\mathrm{C} 2$ & $-0.5(2)$ & $\mathrm{C} 3-\mathrm{N} 2-\mathrm{C} 12-\mathrm{C} 17$ & $-52.6(3)$ \\
\hline $\mathrm{C} 12-\mathrm{N} 2-\mathrm{C} 3-\mathrm{C} 2$ & $176.8(2)$ & $\mathrm{N} 1-\mathrm{N} 2-\mathrm{C} 12-\mathrm{C} 13$ & $-53.0(3)$ \\
\hline $\mathrm{N} 1-\mathrm{N} 2-\mathrm{C} 3-\mathrm{C} 5$ & $176.31(18)$ & $\mathrm{C} 3-\mathrm{N} 2-\mathrm{C} 12-\mathrm{C} 13$ & $129.8(2)$ \\
\hline $\mathrm{C} 12-\mathrm{N} 2-\mathrm{C} 3-\mathrm{C} 5$ & $-6.4(3)$ & $\mathrm{C} 17-\mathrm{C} 12-\mathrm{C} 13-\mathrm{C} 14$ & $0.0(4)$ \\
\hline $\mathrm{C} 1-\mathrm{C} 2-\mathrm{C} 3-\mathrm{N} 2$ & $0.5(2)$ & $\mathrm{N} 2-\mathrm{C} 12-\mathrm{C} 13-\mathrm{C} 14$ & $177.6(2)$ \\
\hline $\mathrm{C} 1-\mathrm{C} 2-\mathrm{C} 3-\mathrm{C} 5$ & $-176.1(2)$ & $\mathrm{C} 12-\mathrm{C} 13-\mathrm{C} 14-\mathrm{C} 15$ & $-0.5(4)$ \\
\hline $\mathrm{N} 1-\mathrm{C} 1-\mathrm{C} 4-\mathrm{F} 2 \mathrm{~A}$ & $75.1(12)$ & $\mathrm{C} 13-\mathrm{C} 14-\mathrm{C} 15-\mathrm{C} 16$ & $0.7(4)$ \\
\hline $\mathrm{C} 2-\mathrm{C} 1-\mathrm{C} 4-\mathrm{F} 2 \mathrm{~A}$ & $-103.1(12)$ & $\mathrm{C} 13-\mathrm{C} 14-\mathrm{C} 15-\mathrm{S} 1$ & $179.24(19)$ \\
\hline $\mathrm{N} 1-\mathrm{C} 1-\mathrm{C} 4-\mathrm{F} 3 \mathrm{~A}$ & $-159.1(11)$ & $\mathrm{O} 1-\mathrm{S} 1-\mathrm{C} 15-\mathrm{C} 16$ & $-161.84(19)$ \\
\hline $\mathrm{C} 2-\mathrm{C} 1-\mathrm{C} 4-\mathrm{F} 3 \mathrm{~A}$ & $22.7(11)$ & $\mathrm{O} 2-\mathrm{S} 1-\mathrm{C} 15-\mathrm{C} 16$ & $-31.5(2)$ \\
\hline $\mathrm{N} 1-\mathrm{C} 1-\mathrm{C} 4-\mathrm{F} 3$ & $158.1(7)$ & $\mathrm{N} 3-\mathrm{S} 1-\mathrm{C} 15-\mathrm{C} 16$ & $83.4(2)$ \\
\hline $\mathrm{C} 2-\mathrm{C} 1-\mathrm{C} 4-\mathrm{F} 3$ & $-20.2(8)$ & $\mathrm{O} 1-\mathrm{S} 1-\mathrm{C} 15-\mathrm{C} 14$ & $19.6(2)$ \\
\hline $\mathrm{N} 1-\mathrm{C} 1-\mathrm{C} 4-\mathrm{F} 2$ & $39.8(7)$ & $\mathrm{O} 2-\mathrm{S} 1-\mathrm{C} 15-\mathrm{C} 14$ & $150.0(2)$ \\
\hline $\mathrm{C} 2-\mathrm{C} 1-\mathrm{C} 4-\mathrm{F} 2$ & $-138.4(6)$ & $\mathrm{N} 3-\mathrm{S} 1-\mathrm{C} 15-\mathrm{C} 14$ & $-95.2(2)$ \\
\hline $\mathrm{N} 1-\mathrm{C} 1-\mathrm{C} 4-\mathrm{F} 1$ & $-82.2(10)$ & $\mathrm{C} 14-\mathrm{C} 15-\mathrm{C} 16-\mathrm{C} 17$ & $-0.4(3)$ \\
\hline $\mathrm{C} 2-\mathrm{C} 1-\mathrm{C} 4-\mathrm{F} 1$ & $99.6(10)$ & $\mathrm{S} 1-\mathrm{C} 15-\mathrm{C} 16-\mathrm{C} 17$ & $-179.00(17)$ \\
\hline $\mathrm{N} 1-\mathrm{C} 1-\mathrm{C} 4-\mathrm{F} 1 \mathrm{~A}$ & $-42.8(8)$ & $\mathrm{C} 13-\mathrm{C} 12-\mathrm{C} 17-\mathrm{C} 16$ & $0.2(3)$ \\
\hline $\mathrm{C} 2-\mathrm{C} 1-\mathrm{C} 4-\mathrm{F} 1 \mathrm{~A}$ & $139.0(8)$ & $\mathrm{N} 2-\mathrm{C} 12-\mathrm{C} 17-\mathrm{C} 16$ & $-177.30(19)$ \\
\hline $\mathrm{N} 2-\mathrm{C} 3-\mathrm{C} 5-\mathrm{C} 10$ & $143.2(2)$ & $\mathrm{C} 15-\mathrm{C} 16-\mathrm{C} 17-\mathrm{C} 12$ & $0.0(3)$ \\
\hline $\mathrm{C} 2-\mathrm{C} 3-\mathrm{C} 5-\mathrm{C} 10$ & $-40.8(3)$ & $\mathrm{C} 5 \mathrm{~S}-\mathrm{N} 2 \mathrm{~S}-\mathrm{C} 1 \mathrm{~S}-\mathrm{O} 1 \mathrm{~S}$ & $153.9(3)$ \\
\hline $\mathrm{N} 2-\mathrm{C} 3-\mathrm{C} 5-\mathrm{C} 6$ & $-39.8(3)$ & $\mathrm{C} 4 \mathrm{~S}-\mathrm{N} 2 \mathrm{~S}-\mathrm{C} 1 \mathrm{~S}-\mathrm{O} 1 \mathrm{~S}$ & $-5.7(4)$ \\
\hline $\mathrm{C} 2-\mathrm{C} 3-\mathrm{C} 5-\mathrm{C} 6$ & $136.2(2)$ & $\mathrm{C} 5 \mathrm{~S}-\mathrm{N} 2 \mathrm{~S}-\mathrm{C} 1 \mathrm{~S}-\mathrm{N} 1 \mathrm{~S}$ & $-24.3(4)$ \\
\hline $\mathrm{C} 10-\mathrm{C} 5-\mathrm{C} 6-\mathrm{C} 7$ & $-0.2(3)$ & $\mathrm{C} 4 \mathrm{~S}-\mathrm{N} 2 \mathrm{~S}-\mathrm{C} 1 \mathrm{~S}-\mathrm{N} 1 \mathrm{~S}$ & $176.1(2)$ \\
\hline $\mathrm{C} 3-\mathrm{C} 5-\mathrm{C} 6-\mathrm{C} 7$ & $-177.20(19)$ & $\mathrm{C} 3 \mathrm{~S}-\mathrm{N} 1 \mathrm{~S}-\mathrm{C} 1 \mathrm{~S}-\mathrm{O} 1 \mathrm{~S}$ & $137.6(3)$ \\
\hline $\mathrm{C} 5-\mathrm{C} 6-\mathrm{C} 7-\mathrm{C} 8$ & $0.1(3)$ & $\mathrm{C} 2 \mathrm{~S}-\mathrm{N} 1 \mathrm{~S}-\mathrm{C} 1 \mathrm{~S}-\mathrm{O} 1 \mathrm{~S}$ & $-8.5(4)$ \\
\hline $\mathrm{C} 6-\mathrm{C} 7-\mathrm{C} 8-\mathrm{C} 9$ & $-0.3(3)$ & $\mathrm{C} 3 \mathrm{~S}-\mathrm{N} 1 \mathrm{~S}-\mathrm{C} 1 \mathrm{~S}-\mathrm{N} 2 \mathrm{~S}$ & $-44.2(4)$ \\
\hline $\mathrm{C} 6-\mathrm{C} 7-\mathrm{C} 8-\mathrm{C} 11$ & $-179.6(2)$ & $\mathrm{C} 2 \mathrm{~S}-\mathrm{N} 1 \mathrm{~S}-\mathrm{C} 1 \mathrm{~S}-\mathrm{N} 2 \mathrm{~S}$ & $169.7(3)$ \\
\hline
\end{tabular}


Hydrogen-bond geometry $\left(\AA,{ }^{\circ}\right)$

\begin{tabular}{lllll}
\hline$D-\mathrm{H} \cdots A$ & $D-\mathrm{H}$ & $\mathrm{H} \cdots A$ & $D \cdots A$ & $D-\mathrm{H} \cdots A$ \\
\hline $\mathrm{N} 3-\mathrm{H} 1 N \cdots \mathrm{O} 1 S$ & $0.87(1)$ & $2.01(1)$ & $2.872(3)$ & $168(2)$ \\
$\mathrm{N} 3-\mathrm{H} 2 N \cdots \mathrm{O} 1 S^{\mathrm{i}}$ & $0.88(1)$ & $2.10(1)$ & $2.955(3)$ & $164(2)$ \\
\hline
\end{tabular}

Symmetry code: (i) $-x+1,-y+1,-z+1$.

4-[5-(4-Methylphenyl)-3-(trifluoromethyl)pyrazol-1-yl] benzenesulfonamide 1,3-

dimethyltetrahydropyrimidin-2(1H)-one monosolvate (5)

Crystal data

$\mathrm{C}_{17} \mathrm{H}_{14} \mathrm{~F}_{3} \mathrm{~N}_{3} \mathrm{O}_{2} \mathrm{~S} \cdot \mathrm{C}_{6} \mathrm{H}_{12} \mathrm{~N}_{2} \mathrm{O}$

$M_{r}=509.55$

Monoclinic, $P 2{ }_{1} / c$

$a=12.4495(17) \AA$

$b=8.7822(13) \AA$

$c=22.656(3) \AA$

$\beta=97.861(5)^{\circ}$

$V=2453.9(6) \AA^{3}$

$Z=4$

$F(000)=1064$

$D_{\mathrm{x}}=1.379 \mathrm{Mg} \mathrm{m}^{-3}$

$\mathrm{Cu} K \alpha$ radiation, $\lambda=1.54178 \AA$

Cell parameters from 9925 reflections

$\theta=3.6-66.6^{\circ}$

$\mu=1.68 \mathrm{~mm}^{-1}$

$T=298 \mathrm{~K}$

Block, colourless

$0.20 \times 0.20 \times 0.18 \mathrm{~mm}$

\section{Data collection}

Bruker D8-QUEST PHOTON-100

diffractometer

Radiation source: Incoatec $\mathrm{I} \mu \mathrm{S} \mathrm{Cu}$ microsource

8 independent reflections

3733 reflections with $I>2 \sigma(I)$

$\omega$ and $\varphi$-scans

$R_{\text {int }}=0.031$

Absorption correction: multi-scan

$\theta_{\max }=66.9^{\circ}, \theta_{\min }=3.6^{\circ}$

$h=-14 \rightarrow 14$

(SADABS; Bruker, 2016)

$k=-10 \rightarrow 10$

$T_{\min }=0.526, T_{\max }=0.753$

$l=-26 \rightarrow 26$

25201 measured reflections

\section{Refinement}

Refinement on $F^{2}$

Least-squares matrix: full

$R\left[F^{2}>2 \sigma\left(F^{2}\right)\right]=0.040$

$w R\left(F^{2}\right)=0.118$

$S=1.02$

4318 reflections

364 parameters

29 restraints

Primary atom site location: dual
Secondary atom site location: difference Fourier map

Hydrogen site location: mixed

$\mathrm{H}$ atoms treated by a mixture of independent and constrained refinement

$w=1 /\left[\sigma^{2}\left(F_{0}^{2}\right)+(0.0598 P)^{2}+0.786 P\right]$

where $P=\left(F_{\mathrm{o}}^{2}+2 F_{\mathrm{c}}{ }^{2}\right) / 3$

$(\Delta / \sigma)_{\max }=0.001$

$\Delta \rho_{\max }=0.25$ e $\AA^{-3}$

$\Delta \rho_{\min }=-0.21$ e $\AA^{-3}$

Special details

Geometry. All esds (except the esd in the dihedral angle between two 1.s. planes) are estimated using the full covariance matrix. The cell esds are taken into account individually in the estimation of esds in distances, angles and torsion angles; correlations between esds in cell parameters are only used when they are defined by crystal symmetry. An approximate (isotropic) treatment of cell esds is used for estimating esds involving l.s. planes.

Fractional atomic coordinates and isotropic or equivalent isotropic displacement parameters $\left(\AA^{2}\right)$

\begin{tabular}{llllll}
\hline & $x$ & $y$ & $z$ & $U_{\text {iso }} * U_{\text {eq }}$ & Occ. $(<1)$ \\
\hline S1 & $0.41794(4)$ & $0.72483(6)$ & $0.57289(2)$ & $0.06125(17)$ &
\end{tabular}




\begin{tabular}{|c|c|c|c|c|c|}
\hline O1 & $0.38898(13)$ & $0.6787(2)$ & $0.62889(7)$ & $0.0869(5)$ & \\
\hline $\mathrm{O} 2$ & $0.37005(12)$ & $0.85770(18)$ & $0.54431(8)$ & $0.0818(5)$ & \\
\hline $\mathrm{N} 1$ & $0.94062(13)$ & $0.86685(19)$ & $0.66122(7)$ & $0.0622(4)$ & \\
\hline $\mathrm{N} 2$ & $0.89648(12)$ & $0.81202(17)$ & $0.60722(7)$ & $0.0542(4)$ & \\
\hline N3 & $0.39091(13)$ & $0.5856(2)$ & $0.52759(8)$ & $0.0620(4)$ & \\
\hline $\mathrm{H} 1 \mathrm{~N}$ & $0.4063(19)$ & $0.611(2)$ & $0.4926(6)$ & $0.083(7)^{*}$ & \\
\hline $\mathrm{H} 2 \mathrm{~N}$ & $0.4186(19)$ & $0.5013(18)$ & $0.5434(9)$ & $0.087(8)^{*}$ & \\
\hline $\mathrm{C} 1$ & $1.04450(16)$ & $0.8842(2)$ & $0.65569(9)$ & $0.0611(5)$ & \\
\hline $\mathrm{C} 2$ & $1.06875(15)$ & $0.8419(2)$ & $0.59995(9)$ & $0.0595(5)$ & \\
\hline $\mathrm{H} 2 \mathrm{~A}$ & 1.136063 & 0.844931 & 0.586550 & $0.071 *$ & \\
\hline $\mathrm{C} 3$ & $0.97180(14)$ & $0.79438(19)$ & $0.56875(8)$ & $0.0508(4)$ & \\
\hline $\mathrm{C} 4$ & $1.1196(2)$ & $0.9403(3)$ & $0.70779(11)$ & $0.0805(7)$ & \\
\hline $\mathrm{F} 1$ & $1.1215(13)$ & $1.0863(6)$ & $0.7165(5)$ & $0.146(4)$ & $0.537(19)$ \\
\hline $\mathrm{F} 2$ & $1.1088(6)$ & $0.8746(13)$ & $0.7582(3)$ & $0.106(2)$ & 0.537 (19) \\
\hline F3 & $1.2216(4)$ & $0.9050(16)$ & $0.7002(4)$ & $0.123(3)$ & $0.537(19)$ \\
\hline F1A & $1.0755(7)$ & $1.0643(9)$ & $0.7306(4)$ & $0.112(2)$ & $0.463(19)$ \\
\hline $\mathrm{F} 2 \mathrm{~A}$ & $1.1290(12)$ & $0.8452(12)$ & $0.7514(5)$ & $0.140(4)$ & $0.463(19)$ \\
\hline F3A & $1.2113(8)$ & $0.981(2)$ & $0.6962(5)$ & $0.162(5)$ & $0.463(19)$ \\
\hline $\mathrm{C} 5$ & $0.94815(13)$ & $0.7296(2)$ & $0.50877(8)$ & $0.0490(4)$ & \\
\hline $\mathrm{C} 6$ & $0.87851(13)$ & $0.6065(2)$ & $0.49599(8)$ & $0.0506(4)$ & \\
\hline H6A & 0.843189 & 0.565111 & 0.525859 & $0.061 *$ & \\
\hline $\mathrm{C} 7$ & $0.86134(14)$ & $0.5453(2)$ & $0.43937(8)$ & $0.0539(4)$ & \\
\hline H7A & 0.814361 & 0.463082 & 0.431819 & $0.065^{*}$ & \\
\hline $\mathrm{C} 8$ & $0.91219(15)$ & $0.6029(2)$ & $0.39355(8)$ & $0.0579(5)$ & \\
\hline $\mathrm{C} 9$ & $0.98180(17)$ & $0.7245(2)$ & $0.40657(9)$ & $0.0642(5)$ & \\
\hline H9A & 1.017106 & 0.765364 & 0.376597 & $0.077^{*}$ & \\
\hline $\mathrm{C} 10$ & $1.00027(16)$ & $0.7868(2)$ & $0.46305(9)$ & $0.0597(5)$ & \\
\hline $\mathrm{H} 10 \mathrm{~A}$ & 1.048129 & 0.868048 & 0.470546 & $0.072 *$ & \\
\hline $\mathrm{C} 11$ & $0.8913(2)$ & $0.5355(3)$ & $0.33191(10)$ & $0.0823(7)$ & \\
\hline H11A & 0.841042 & 0.452615 & 0.331654 & $0.123^{*}$ & \\
\hline H11B & 0.861432 & 0.612155 & 0.304201 & $0.123^{*}$ & \\
\hline $\mathrm{H} 11 \mathrm{C}$ & 0.958260 & 0.498922 & 0.320603 & $0.123 *$ & \\
\hline $\mathrm{C} 12$ & $0.78175(14)$ & $0.7888(2)$ & $0.59761(8)$ & $0.0505(4)$ & \\
\hline $\mathrm{C} 13$ & $0.73524(16)$ & $0.6991(2)$ & $0.63745(8)$ & $0.0598(5)$ & \\
\hline H13A & 0.778399 & 0.652147 & 0.669010 & $0.072 *$ & \\
\hline $\mathrm{C} 14$ & $0.62451(16)$ & $0.6800(2)$ & $0.62994(9)$ & $0.0611(5)$ & \\
\hline $\mathrm{H} 14 \mathrm{~A}$ & 0.592406 & 0.619688 & 0.656376 & $0.073 *$ & \\
\hline $\mathrm{C} 15$ & $0.56091(15)$ & $0.7510(2)$ & $0.58288(8)$ & $0.0523(4)$ & \\
\hline $\mathrm{C} 16$ & $0.60771(14)$ & $0.8393(2)$ & $0.54282(8)$ & $0.0534(4)$ & \\
\hline H16A & 0.564691 & 0.885626 & 0.511055 & $0.064 *$ & \\
\hline $\mathrm{C} 17$ & $0.71903(14)$ & $0.8583(2)$ & $0.55034(8)$ & $0.0526(4)$ & \\
\hline H17A & 0.751369 & 0.917624 & 0.523677 & $0.063 *$ & \\
\hline O1S & $0.47338(11)$ & $0.66985(18)$ & $0.41995(6)$ & $0.0705(4)$ & \\
\hline N1S & $0.54014(13)$ & $0.7997(2)$ & $0.34753(8)$ & $0.0687(5)$ & \\
\hline $\mathrm{N} 2 \mathrm{~S}$ & $0.35615(13)$ & $0.7975(2)$ & $0.35315(7)$ & $0.0677(5)$ & \\
\hline $\mathrm{C} 1 \mathrm{~S}$ & $0.45703(15)$ & $0.7530(2)$ & $0.37519(8)$ & $0.0549(4)$ & \\
\hline $\mathrm{C} 2 \mathrm{~S}$ & $0.5267(2)$ & $0.8980(4)$ & $0.29596(12)$ & $0.1010(9)$ & $0.584(16)$ \\
\hline $\mathrm{H} 1 \mathrm{~S}$ & 0.574357 & 0.864441 & 0.268074 & $0.121 *$ & $0.584(16)$ \\
\hline
\end{tabular}




\begin{tabular}{|c|c|c|c|c|c|}
\hline $\mathrm{H} 2 \mathrm{~S}$ & 0.547167 & 1.001104 & 0.308134 & $0.121 *$ & $0.584(16)$ \\
\hline C3S & $0.4159(5)$ & $0.8973(14)$ & 0.2668 & $0.091(2)$ & $0.584(16)$ \\
\hline $\mathrm{H} 3 \mathrm{~S}$ & 0.406119 & 0.984954 & 0.240594 & $0.109 *$ & $0.584(16)$ \\
\hline $\mathrm{H} 4 \mathrm{~S}$ & 0.405910 & 0.807169 & 0.241903 & $0.109^{*}$ & $0.584(16)$ \\
\hline $\mathrm{C} 4 \mathrm{~S}$ & $0.3331(2)$ & $0.8996(4)$ & $0.30334(14)$ & $0.1001(9)$ & $0.584(16)$ \\
\hline $\mathrm{H} 5 \mathrm{~S}$ & 0.265038 & 0.870624 & 0.279950 & $0.120 *$ & $0.584(16)$ \\
\hline H6S & 0.325220 & 1.002247 & 0.317936 & $0.120 *$ & $0.584(16)$ \\
\hline $\mathrm{C} 2 \mathrm{SA}$ & $0.5267(2)$ & $0.8980(4)$ & $0.29596(12)$ & $0.1010(9)$ & $0.416(16)$ \\
\hline $\mathrm{H} 7 \mathrm{~S}$ & 0.583895 & 0.973801 & 0.301486 & $0.121^{*}$ & $0.416(16)$ \\
\hline H8S & 0.538350 & 0.837141 & 0.261690 & $0.121^{*}$ & $0.416(16)$ \\
\hline C3SA & $0.4282(8)$ & $0.9749(14)$ & $0.2818(5)$ & $0.085(3)$ & $0.416(16)$ \\
\hline H9S & 0.413220 & 0.985276 & 0.238792 & $0.102^{*}$ & $0.416(16)$ \\
\hline H10S & 0.435769 & 1.076536 & 0.298529 & $0.102 *$ & $0.416(16)$ \\
\hline C4SA & $0.3331(2)$ & $0.8996(4)$ & $0.30334(14)$ & $0.1001(9)$ & $0.416(16)$ \\
\hline $\mathrm{H} 11 \mathrm{~S}$ & 0.284975 & 0.978559 & 0.314179 & $0.120 *$ & $0.416(16)$ \\
\hline $\mathrm{H} 12 \mathrm{~S}$ & 0.293977 & 0.843185 & 0.270375 & $0.120 *$ & $0.416(16)$ \\
\hline $\mathrm{C} 5 \mathrm{~S}$ & 0.65001 (19) & $0.7594(4)$ & $0.37258(13)$ & $0.0941(8)$ & \\
\hline H13S & 0.700024 & 0.800549 & 0.348052 & $0.141 *$ & \\
\hline $\mathrm{H} 14 \mathrm{~S}$ & 0.665658 & 0.800556 & 0.412086 & $0.141 *$ & \\
\hline H15S & 0.657044 & 0.650610 & 0.374190 & $0.141 *$ & \\
\hline C6S & $0.26640(17)$ & $0.7537(3)$ & $0.38401(12)$ & $0.0850(7)$ & \\
\hline H16S & 0.200077 & 0.793538 & 0.363098 & $0.128 *$ & \\
\hline H17S & 0.262107 & 0.644691 & 0.385612 & $0.128 *$ & \\
\hline H18S & 0.277552 & 0.793959 & 0.423776 & $0.128 *$ & \\
\hline
\end{tabular}

Atomic displacement parameters $\left(\AA^{2}\right)$

\begin{tabular}{lllllll}
\hline & $U^{11}$ & $U^{22}$ & $U^{33}$ & $U^{12}$ & $U^{13}$ & $U^{23}$ \\
\hline S1 & $0.0469(3)$ & $0.0736(3)$ & $0.0663(3)$ & $0.0024(2)$ & $0.0187(2)$ & $0.0018(2)$ \\
O1 & $0.0710(9)$ & $0.1265(14)$ & $0.0697(9)$ & $-0.0083(9)$ & $0.0335(7)$ & $-0.0009(9)$ \\
O2 & $0.0571(8)$ & $0.0745(10)$ & $0.1146(12)$ & $0.0166(7)$ & $0.0140(8)$ & $0.0083(9)$ \\
N1 & $0.0612(10)$ & $0.0664(10)$ & $0.0561(9)$ & $-0.0045(8)$ & $-0.0021(7)$ & $-0.0045(8)$ \\
N2 & $0.0474(8)$ & $0.0569(9)$ & $0.0562(8)$ & $-0.0003(7)$ & $-0.0001(7)$ & $-0.0036(7)$ \\
N3 & $0.0529(9)$ & $0.0713(11)$ & $0.0636(10)$ & $-0.0069(8)$ & $0.0145(8)$ & $0.0069(9)$ \\
C1 & $0.0593(11)$ & $0.0561(11)$ & $0.0640(11)$ & $-0.0075(9)$ & $-0.0054(9)$ & $0.0017(9)$ \\
C2 & $0.0486(10)$ & $0.0561(11)$ & $0.0717(12)$ & $-0.0060(8)$ & $0.0007(9)$ & $0.0029(9)$ \\
C3 & $0.0467(9)$ & $0.0441(9)$ & $0.0604(10)$ & $0.0005(7)$ & $0.0030(8)$ & $0.0040(8)$ \\
C4 & $0.0760(16)$ & $0.0854(17)$ & $0.0740(15)$ & $-0.0148(14)$ & $-0.0119(12)$ & $-0.0004(13)$ \\
F1 & $0.196(9)$ & $0.068(2)$ & $0.142(6)$ & $-0.021(4)$ & $-0.092(6)$ & $-0.013(3)$ \\
F2 & $0.082(3)$ & $0.170(7)$ & $0.061(2)$ & $-0.031(3)$ & $-0.009(2)$ & $0.013(3)$ \\
F3 & $0.057(3)$ & $0.182(7)$ & $0.122(4)$ & $-0.022(3)$ & $-0.020(2)$ & $-0.041(4)$ \\
F1A & $0.129(5)$ & $0.109(4)$ & $0.092(3)$ & $-0.037(3)$ & $-0.013(3)$ & $-0.034(3)$ \\
F2A & $0.171(9)$ & $0.108(4)$ & $0.115(7)$ & $0.005(4)$ & $-0.070(5)$ & $0.025(4)$ \\
F3A & $0.120(7)$ & $0.245(13)$ & $0.124(5)$ & $-0.124(8)$ & $0.024(5)$ & $-0.056(7)$ \\
C5 & $0.0396(8)$ & $0.0480(9)$ & $0.0588(10)$ & $0.0041(7)$ & $0.0045(7)$ & $0.0030(8)$ \\
C6 & $0.0426(9)$ & $0.0489(9)$ & $0.0611(10)$ & $0.0006(7)$ & $0.0106(7)$ & $0.0009(8)$ \\
C7 & $0.0417(9)$ & $0.0531(10)$ & $0.0666(11)$ & $0.0024(8)$ & $0.0061(8)$ & $-0.0053(8)$ \\
C8 & $0.0500(10)$ & $0.0625(11)$ & $0.0611(11)$ & $0.0122(9)$ & $0.0074(8)$ & $-0.0015(9)$ \\
& & & & &
\end{tabular}


supporting information

\begin{tabular}{lllllll} 
C9 & $0.0592(11)$ & $0.0728(13)$ & $0.0635(12)$ & $0.0017(10)$ & $0.0187(9)$ & $0.0100(10)$ \\
C10 & $0.0515(10)$ & $0.0564(11)$ & $0.0712(12)$ & $-0.0069(8)$ & $0.0088(9)$ & $0.0048(9)$ \\
C11 & $0.0911(16)$ & $0.0916(17)$ & $0.0652(13)$ & $0.0070(13)$ & $0.0144(11)$ & $-0.0116(12)$ \\
C12 & $0.0474(9)$ & $0.0500(9)$ & $0.0540(10)$ & $0.0011(7)$ & $0.0062(7)$ & $-0.0047(8)$ \\
C13 & $0.0597(11)$ & $0.0641(12)$ & $0.0540(10)$ & $0.0018(9)$ & $0.0021(8)$ & $0.0093(9)$ \\
C14 & $0.0606(11)$ & $0.0673(12)$ & $0.0564(10)$ & $-0.0048(9)$ & $0.0119(9)$ & $0.0082(9)$ \\
C15 & $0.0489(10)$ & $0.0545(10)$ & $0.0547(10)$ & $0.0024(8)$ & $0.0113(8)$ & $-0.0018(8)$ \\
C16 & $0.0468(9)$ & $0.0559(10)$ & $0.0574(10)$ & $0.0050(8)$ & $0.0066(8)$ & $0.0041(8)$ \\
C17 & $0.0504(10)$ & $0.0516(10)$ & $0.0565(10)$ & $0.0012(8)$ & $0.0099(8)$ & $0.0062(8)$ \\
O1S & $0.0572(8)$ & $0.0912(10)$ & $0.0638(8)$ & $0.0037(7)$ & $0.0115(6)$ & $0.0201(8)$ \\
N1S & $0.0546(9)$ & $0.0859(12)$ & $0.0663(10)$ & $0.0040(8)$ & $0.0103(8)$ & $0.0207(9)$ \\
N2S & $0.0514(9)$ & $0.0873(12)$ & $0.0622(10)$ & $0.0056(8)$ & $-0.0001(7)$ & $0.0004(9)$ \\
C1S & $0.0515(10)$ & $0.0621(11)$ & $0.0503(10)$ & $0.0026(8)$ & $0.0047(8)$ & $-0.0013(8)$ \\
C2S & $0.0911(18)$ & $0.124(2)$ & $0.0878(17)$ & $-0.0044(17)$ & $0.0131(14)$ & $0.0457(17)$ \\
C3S & $0.113(4)$ & $0.102(5)$ & $0.056(3)$ & $0.016(4)$ & $0.001(2)$ & $0.014(3)$ \\
C4S & $0.0854(18)$ & $0.101(2)$ & $0.105(2)$ & $0.0151(15)$ & $-0.0189(15)$ & $0.0244(16)$ \\
C2SA & $0.0911(18)$ & $0.124(2)$ & $0.0878(17)$ & $-0.0044(17)$ & $0.0131(14)$ & $0.0457(17)$ \\
C3SA & $0.106(5)$ & $0.073(5)$ & $0.073(5)$ & $0.008(4)$ & $0.007(4)$ & $0.017(4)$ \\
C4SA & $0.0854(18)$ & $0.101(2)$ & $0.105(2)$ & $0.0151(15)$ & $-0.0189(15)$ & $0.0244(16)$ \\
C5S & $0.0536(12)$ & $0.132(2)$ & $0.0985(18)$ & $0.0083(13)$ & $0.0174(12)$ & $0.0368(17)$ \\
C6S & $0.0485(12)$ & $0.1091(19)$ & $0.0972(17)$ & $0.0005(12)$ & $0.0088(11)$ & $-0.0092(15)$ \\
& & & & & & \\
\hline
\end{tabular}

Geometric parameters $\left(\AA,{ }^{\circ}\right)$

\begin{tabular}{llll}
\hline $\mathrm{S} 1-\mathrm{O} 1$ & $1.4246(15)$ & $\mathrm{C} 13-\mathrm{H} 13 \mathrm{~A}$ & 0.9300 \\
$\mathrm{~S} 1-\mathrm{O} 2$ & $1.4250(16)$ & $\mathrm{C} 14-\mathrm{C} 15$ & $1.386(3)$ \\
$\mathrm{S} 1-\mathrm{N} 3$ & $1.6020(19)$ & $\mathrm{C} 14-\mathrm{H} 14 \mathrm{~A}$ & 0.9300 \\
$\mathrm{~S} 1-\mathrm{C} 15$ & $1.7783(19)$ & $\mathrm{C} 15-\mathrm{C} 16$ & $1.382(3)$ \\
$\mathrm{N} 1-\mathrm{C} 1$ & $1.325(3)$ & $\mathrm{C} 16-\mathrm{C} 17$ & $1.383(3)$ \\
$\mathrm{N} 1-\mathrm{N} 2$ & $1.359(2)$ & $\mathrm{C} 16-\mathrm{H} 16 \mathrm{~A}$ & 0.9300 \\
$\mathrm{~N} 2-\mathrm{C} 3$ & $1.373(2)$ & $\mathrm{C} 17-\mathrm{H} 17 \mathrm{~A}$ & 0.9300 \\
$\mathrm{~N} 2-\mathrm{C} 12$ & $1.430(2)$ & $\mathrm{O} 1 \mathrm{~S}-\mathrm{C} 1 \mathrm{~S}$ & $1.244(2)$ \\
$\mathrm{N} 3-\mathrm{H} 1 \mathrm{~N}$ & $0.869(9)$ & $\mathrm{N} 1 \mathrm{~S}-\mathrm{C} 1 \mathrm{~S}$ & $1.345(2)$ \\
$\mathrm{N} 3-\mathrm{H} 2 \mathrm{~N}$ & $0.873(9)$ & $\mathrm{N} 1 \mathrm{~S}-\mathrm{C} 2 \mathrm{SA}$ & $1.444(3)$ \\
$\mathrm{C} 1-\mathrm{C} 2$ & $1.389(3)$ & $\mathrm{N} 1 \mathrm{~S}-\mathrm{C} 2 \mathrm{~S}$ & $1.444(3)$ \\
$\mathrm{C} 1-\mathrm{C} 4$ & $1.486(3)$ & $\mathrm{N} 1 \mathrm{~S}-\mathrm{C} 5 \mathrm{~S}$ & $1.451(3)$ \\
$\mathrm{C} 2-\mathrm{C} 3$ & $1.377(3)$ & $\mathrm{N} 2 \mathrm{~S}-\mathrm{C} 1 \mathrm{~S}$ & $1.344(2)$ \\
$\mathrm{C} 2-\mathrm{H} 2 \mathrm{~A}$ & 0.9300 & $\mathrm{~N} 2 \mathrm{~S}-\mathrm{C} 4 \mathrm{SA}$ & $1.439(3)$ \\
$\mathrm{C} 3-\mathrm{C} 5$ & $1.466(3)$ & $\mathrm{N} 2 \mathrm{~S}-\mathrm{C} 4 \mathrm{~S}$ & $1.439(3)$ \\
$\mathrm{C} 4-\mathrm{F} 3 \mathrm{~S}$ & $1.259(6)$ & $\mathrm{C} 2 \mathrm{~S}-\mathrm{C} 6 \mathrm{~S}$ & $1.449(3)$ \\
$\mathrm{C} 4-\mathrm{F} 2 \mathrm{~S}$ & $1.286(7)$ & $\mathrm{C} 2 \mathrm{~S}-\mathrm{H} 1 \mathrm{~S}$ & $1.446(6)$ \\
$\mathrm{C} 4-\mathrm{F} 1$ & $1.297(5)$ & $\mathrm{C} 2 \mathrm{~S}-\mathrm{H} 2 \mathrm{~S}$ & 0.9700 \\
$\mathrm{C} 4-\mathrm{F} 2$ & $1.302(6)$ & $\mathrm{C} 3 \mathrm{~S}-\mathrm{C} 4 \mathrm{~S}$ & 0.9700 \\
$\mathrm{C} 4-\mathrm{F} 3$ & $1.341(6)$ & $\mathrm{C} 3 \mathrm{~S}-\mathrm{H} 3 \mathrm{~S}$ & $1.407(6)$ \\
$\mathrm{C} 4-\mathrm{F} 1 \mathrm{~A}$ & $1.353(7)$ & $\mathrm{C} 4 \mathrm{~S}-\mathrm{H} 4 \mathrm{H} 5 \mathrm{~S}$ & 0.9700 \\
$\mathrm{C} 5-\mathrm{C} 10$ & $1.389(3)$ & $\mathrm{C} 4 \mathrm{~S}-\mathrm{H} 6 \mathrm{~S}$ & 0.9700 \\
$\mathrm{C} 5-\mathrm{C} 6$ & $1.391(2)$ & & 0.9700 \\
$\mathrm{C} 6-\mathrm{C} 7$ & $1.380(3)$ & & 0.9700 \\
& &
\end{tabular}




\begin{tabular}{|c|c|c|c|}
\hline C6-H6A & 0.9300 & $\mathrm{C} 2 \mathrm{SA}-\mathrm{C} 3 \mathrm{SA}$ & $1.398(9)$ \\
\hline $\mathrm{C} 7-\mathrm{C} 8$ & $1.384(3)$ & $\mathrm{C} 2 \mathrm{SA}-\mathrm{H} 7 \mathrm{~S}$ & 0.9700 \\
\hline C7-H7A & 0.9300 & $\mathrm{C} 2 \mathrm{SA}-\mathrm{H} 8 \mathrm{~S}$ & 0.9700 \\
\hline $\mathrm{C} 8-\mathrm{C} 9$ & $1.381(3)$ & $\mathrm{C} 3 \mathrm{SA}-\mathrm{C} 4 \mathrm{SA}$ & $1.495(10)$ \\
\hline $\mathrm{C} 8-\mathrm{C} 11$ & $1.506(3)$ & $\mathrm{C} 3 \mathrm{SA}-\mathrm{H} 9 \mathrm{~S}$ & 0.9700 \\
\hline $\mathrm{C} 9-\mathrm{C} 10$ & $1.382(3)$ & $\mathrm{C} 3 \mathrm{SA}-\mathrm{H} 10 \mathrm{~S}$ & 0.9700 \\
\hline C9- $\mathrm{H} 9 \mathrm{~A}$ & 0.9300 & $\mathrm{C} 4 \mathrm{SA}-\mathrm{H} 11 \mathrm{~S}$ & 0.9700 \\
\hline $\mathrm{C} 10-\mathrm{H} 10 \mathrm{~A}$ & 0.9300 & $\mathrm{C} 4 \mathrm{SA}-\mathrm{H} 12 \mathrm{~S}$ & 0.9700 \\
\hline $\mathrm{C} 11-\mathrm{H} 11 \mathrm{~A}$ & 0.9600 & $\mathrm{C} 5 \mathrm{~S}-\mathrm{H} 13 \mathrm{~S}$ & 0.9600 \\
\hline C11-H11B & 0.9600 & $\mathrm{C} 5 \mathrm{~S}-\mathrm{H} 14 \mathrm{~S}$ & 0.9600 \\
\hline $\mathrm{C} 11-\mathrm{H} 11 \mathrm{C}$ & 0.9600 & $\mathrm{C} 5 \mathrm{~S}-\mathrm{H} 15 \mathrm{~S}$ & 0.9600 \\
\hline $\mathrm{C} 12-\mathrm{C} 17$ & $1.379(3)$ & $\mathrm{C} 6 \mathrm{~S}-\mathrm{H} 16 \mathrm{~S}$ & 0.9600 \\
\hline $\mathrm{C} 12-\mathrm{C} 13$ & $1.383(3)$ & $\mathrm{C} 6 \mathrm{~S}-\mathrm{H} 17 \mathrm{~S}$ & 0.9600 \\
\hline $\mathrm{C} 13-\mathrm{C} 14$ & $1.376(3)$ & $\mathrm{C} 6 \mathrm{~S}-\mathrm{H} 18 \mathrm{~S}$ & 0.9600 \\
\hline $\mathrm{O} 1-\mathrm{S} 1-\mathrm{O} 2$ & $119.78(11)$ & $\mathrm{C} 14-\mathrm{C} 15-\mathrm{S} 1$ & $119.76(14)$ \\
\hline $\mathrm{O} 1-\mathrm{S} 1-\mathrm{N} 3$ & $107.27(10)$ & $\mathrm{C} 15-\mathrm{C} 16-\mathrm{C} 17$ & $119.49(17)$ \\
\hline $\mathrm{O} 2-\mathrm{S} 1-\mathrm{N} 3$ & $107.20(10)$ & $\mathrm{C} 15-\mathrm{C} 16-\mathrm{H} 16 \mathrm{~A}$ & 120.3 \\
\hline $\mathrm{O} 1-\mathrm{S} 1-\mathrm{C} 15$ & $107.11(9)$ & $\mathrm{C} 17-\mathrm{C} 16-\mathrm{H} 16 \mathrm{~A}$ & 120.3 \\
\hline $\mathrm{O} 2-\mathrm{S} 1-\mathrm{C} 15$ & $107.54(9)$ & $\mathrm{C} 12-\mathrm{C} 17-\mathrm{C} 16$ & $119.59(17)$ \\
\hline N3-S1-C15 & $107.40(8)$ & $\mathrm{C} 12-\mathrm{C} 17-\mathrm{H} 17 \mathrm{~A}$ & 120.2 \\
\hline $\mathrm{C} 1-\mathrm{N} 1-\mathrm{N} 2$ & $103.60(15)$ & $\mathrm{C} 16-\mathrm{C} 17-\mathrm{H} 17 \mathrm{~A}$ & 120.2 \\
\hline $\mathrm{N} 1-\mathrm{N} 2-\mathrm{C} 3$ & $112.59(15)$ & $\mathrm{C} 1 \mathrm{~S}-\mathrm{N} 1 \mathrm{~S}-\mathrm{C} 2 \mathrm{SA}$ & $123.21(19)$ \\
\hline $\mathrm{N} 1-\mathrm{N} 2-\mathrm{C} 12$ & $117.39(15)$ & $\mathrm{C} 1 \mathrm{~S}-\mathrm{N} 1 \mathrm{~S}-\mathrm{C} 2 \mathrm{~S}$ & $123.21(19)$ \\
\hline $\mathrm{C} 3-\mathrm{N} 2-\mathrm{C} 12$ & $129.94(15)$ & $\mathrm{C} 1 \mathrm{~S}-\mathrm{N} 1 \mathrm{~S}-\mathrm{C} 5 \mathrm{~S}$ & $119.20(17)$ \\
\hline $\mathrm{S} 1-\mathrm{N} 3-\mathrm{H} 1 \mathrm{~N}$ & $109.8(16)$ & $\mathrm{C} 2 \mathrm{SA}-\mathrm{N} 1 \mathrm{~S}-\mathrm{C} 5 \mathrm{~S}$ & $117.44(19)$ \\
\hline $\mathrm{S} 1-\mathrm{N} 3-\mathrm{H} 2 \mathrm{~N}$ & $110.4(17)$ & $\mathrm{C} 2 \mathrm{~S}-\mathrm{N} 1 \mathrm{~S}-\mathrm{C} 5 \mathrm{~S}$ & $117.44(19)$ \\
\hline $\mathrm{H} 1 \mathrm{~N}-\mathrm{N} 3-\mathrm{H} 2 \mathrm{~N}$ & $117.6(15)$ & $\mathrm{C} 1 \mathrm{~S}-\mathrm{N} 2 \mathrm{~S}-\mathrm{C} 4 \mathrm{SA}$ & $123.2(2)$ \\
\hline $\mathrm{N} 1-\mathrm{C} 1-\mathrm{C} 2$ & $113.06(16)$ & $\mathrm{C} 1 \mathrm{~S}-\mathrm{N} 2 \mathrm{~S}-\mathrm{C} 4 \mathrm{~S}$ & $123.2(2)$ \\
\hline $\mathrm{N} 1-\mathrm{C} 1-\mathrm{C} 4$ & $118.6(2)$ & $\mathrm{C} 1 \mathrm{~S}-\mathrm{N} 2 \mathrm{~S}-\mathrm{C} 6 \mathrm{~S}$ & $119.13(18)$ \\
\hline $\mathrm{C} 2-\mathrm{C} 1-\mathrm{C} 4$ & $128.3(2)$ & $\mathrm{C} 4 \mathrm{SA}-\mathrm{N} 2 \mathrm{~S}-\mathrm{C} 6 \mathrm{~S}$ & $117.4(2)$ \\
\hline $\mathrm{C} 3-\mathrm{C} 2-\mathrm{C} 1$ & $105.28(17)$ & $\mathrm{C} 4 \mathrm{~S}-\mathrm{N} 2 \mathrm{~S}-\mathrm{C} 6 \mathrm{~S}$ & $117.4(2)$ \\
\hline $\mathrm{C} 3-\mathrm{C} 2-\mathrm{H} 2 \mathrm{~A}$ & 127.4 & $\mathrm{O} 1 \mathrm{~S}-\mathrm{C} 1 \mathrm{~S}-\mathrm{N} 2 \mathrm{~S}$ & $120.91(18)$ \\
\hline $\mathrm{C} 1-\mathrm{C} 2-\mathrm{H} 2 \mathrm{~A}$ & 127.4 & $\mathrm{O} 1 \mathrm{~S}-\mathrm{C} 1 \mathrm{~S}-\mathrm{N} 1 \mathrm{~S}$ & $120.55(17)$ \\
\hline $\mathrm{N} 2-\mathrm{C} 3-\mathrm{C} 2$ & $105.47(16)$ & $\mathrm{N} 2 \mathrm{~S}-\mathrm{C} 1 \mathrm{~S}-\mathrm{N} 1 \mathrm{~S}$ & $118.54(17)$ \\
\hline $\mathrm{N} 2-\mathrm{C} 3-\mathrm{C} 5$ & $124.35(15)$ & $\mathrm{N} 1 \mathrm{~S}-\mathrm{C} 2 \mathrm{~S}-\mathrm{C} 3 \mathrm{~S}$ & $111.4(4)$ \\
\hline $\mathrm{C} 2-\mathrm{C} 3-\mathrm{C} 5$ & $130.11(17)$ & $\mathrm{N} 1 \mathrm{~S}-\mathrm{C} 2 \mathrm{~S}-\mathrm{H} 1 \mathrm{~S}$ & 109.4 \\
\hline $\mathrm{F} 3 \mathrm{~A}-\mathrm{C} 4-\mathrm{F} 2 \mathrm{~A}$ & $110.8(7)$ & $\mathrm{C} 3 \mathrm{~S}-\mathrm{C} 2 \mathrm{~S}-\mathrm{H} 1 \mathrm{~S}$ & 109.4 \\
\hline $\mathrm{F} 1-\mathrm{C} 4-\mathrm{F} 2$ & $107.8(6)$ & $\mathrm{N} 1 \mathrm{~S}-\mathrm{C} 2 \mathrm{~S}-\mathrm{H} 2 \mathrm{~S}$ & 109.4 \\
\hline $\mathrm{F} 1-\mathrm{C} 4-\mathrm{F} 3$ & $104.4(5)$ & $\mathrm{C} 3 \mathrm{~S}-\mathrm{C} 2 \mathrm{~S}-\mathrm{H} 2 \mathrm{~S}$ & 109.4 \\
\hline $\mathrm{F} 2-\mathrm{C} 4-\mathrm{F} 3$ & $102.9(5)$ & $\mathrm{H} 1 \mathrm{~S}-\mathrm{C} 2 \mathrm{~S}-\mathrm{H} 2 \mathrm{~S}$ & 108.0 \\
\hline $\mathrm{F} 3 \mathrm{~A}-\mathrm{C} 4-\mathrm{F} 1 \mathrm{~A}$ & $106.0(6)$ & $\mathrm{C} 4 \mathrm{~S}-\mathrm{C} 3 \mathrm{~S}-\mathrm{C} 2 \mathrm{~S}$ & $117.5(5)$ \\
\hline $\mathrm{F} 2 \mathrm{~A}-\mathrm{C} 4-\mathrm{F} 1 \mathrm{~A}$ & $103.2(6)$ & $\mathrm{C} 4 \mathrm{~S}-\mathrm{C} 3 \mathrm{~S}-\mathrm{H} 3 \mathrm{~S}$ & 107.9 \\
\hline $\mathrm{F} 3 \mathrm{~A}-\mathrm{C} 4-\mathrm{C} 1$ & $114.8(5)$ & $\mathrm{C} 2 \mathrm{~S}-\mathrm{C} 3 \mathrm{~S}-\mathrm{H} 3 \mathrm{~S}$ & 107.9 \\
\hline $\mathrm{F} 2 \mathrm{~A}-\mathrm{C} 4-\mathrm{C} 1$ & $112.1(6)$ & $\mathrm{C} 4 \mathrm{~S}-\mathrm{C} 3 \mathrm{~S}-\mathrm{H} 4 \mathrm{~S}$ & 107.9 \\
\hline $\mathrm{F} 1-\mathrm{C} 4-\mathrm{C} 1$ & $116.4(4)$ & $\mathrm{C} 2 \mathrm{~S}-\mathrm{C} 3 \mathrm{~S}-\mathrm{H} 4 \mathrm{~S}$ & 107.9 \\
\hline $\mathrm{F} 2-\mathrm{C} 4-\mathrm{C} 1$ & $114.9(4)$ & $\mathrm{H} 3 \mathrm{~S}-\mathrm{C} 3 \mathrm{~S}-\mathrm{H} 4 \mathrm{~S}$ & 107.2 \\
\hline $\mathrm{F} 3-\mathrm{C} 4-\mathrm{C} 1$ & $109.0(4)$ & $\mathrm{C} 3 \mathrm{~S}-\mathrm{C} 4 \mathrm{~S}-\mathrm{N} 2 \mathrm{~S}$ & $111.6(4)$ \\
\hline
\end{tabular}




\begin{tabular}{|c|c|}
\hline $\mathrm{F} 1 \mathrm{~A}-\mathrm{C} 4-\mathrm{C} 1$ & $109.0(4)$ \\
\hline $\mathrm{C} 10-\mathrm{C} 5-\mathrm{C} 6$ & $117.86(17)$ \\
\hline $\mathrm{C} 10-\mathrm{C} 5-\mathrm{C} 3$ & $119.72(16)$ \\
\hline $\mathrm{C} 6-\mathrm{C} 5-\mathrm{C} 3$ & $122.35(16)$ \\
\hline $\mathrm{C} 7-\mathrm{C} 6-\mathrm{C} 5$ & $120.60(17)$ \\
\hline $\mathrm{C} 7-\mathrm{C} 6-\mathrm{H} 6 \mathrm{~A}$ & 119.7 \\
\hline $\mathrm{C} 5-\mathrm{C} 6-\mathrm{H} 6 \mathrm{~A}$ & 119.7 \\
\hline $\mathrm{C} 6-\mathrm{C} 7-\mathrm{C} 8$ & $121.74(17)$ \\
\hline $\mathrm{C} 6-\mathrm{C} 7-\mathrm{H} 7 \mathrm{~A}$ & 119.1 \\
\hline $\mathrm{C} 8-\mathrm{C} 7-\mathrm{H} 7 \mathrm{~A}$ & 119.1 \\
\hline $\mathrm{C} 9-\mathrm{C} 8-\mathrm{C} 7$ & $117.43(18)$ \\
\hline $\mathrm{C} 9-\mathrm{C} 8-\mathrm{C} 11$ & $121.7(2)$ \\
\hline $\mathrm{C} 7-\mathrm{C} 8-\mathrm{C} 11$ & $120.9(2)$ \\
\hline $\mathrm{C} 8-\mathrm{C} 9-\mathrm{C} 10$ & $121.61(18)$ \\
\hline $\mathrm{C} 8-\mathrm{C} 9-\mathrm{H} 9 \mathrm{~A}$ & 119.2 \\
\hline $\mathrm{C} 10-\mathrm{C} 9-\mathrm{H} 9 \mathrm{~A}$ & 119.2 \\
\hline $\mathrm{C} 9-\mathrm{C} 10-\mathrm{C} 5$ & $120.76(18)$ \\
\hline $\mathrm{C} 9-\mathrm{C} 10-\mathrm{H} 10 \mathrm{~A}$ & 119.6 \\
\hline $\mathrm{C} 5-\mathrm{C} 10-\mathrm{H} 10 \mathrm{~A}$ & 119.6 \\
\hline $\mathrm{C} 8-\mathrm{C} 11-\mathrm{H} 11 \mathrm{~A}$ & 109.5 \\
\hline $\mathrm{C} 8-\mathrm{C} 11-\mathrm{H} 11 \mathrm{~B}$ & 109.5 \\
\hline $\mathrm{H} 11 \mathrm{~A}-\mathrm{C} 11-\mathrm{H} 11 \mathrm{~B}$ & 109.5 \\
\hline $\mathrm{C} 8-\mathrm{C} 11-\mathrm{H} 11 \mathrm{C}$ & 109.5 \\
\hline $\mathrm{H} 11 \mathrm{~A}-\mathrm{C} 11-\mathrm{H} 11 \mathrm{C}$ & 109.5 \\
\hline $\mathrm{H} 11 \mathrm{~B}-\mathrm{C} 11-\mathrm{H} 11 \mathrm{C}$ & 109.5 \\
\hline $\mathrm{C} 17-\mathrm{C} 12-\mathrm{C} 13$ & $121.06(17)$ \\
\hline $\mathrm{C} 17-\mathrm{C} 12-\mathrm{N} 2$ & $120.19(16)$ \\
\hline $\mathrm{C} 13-\mathrm{C} 12-\mathrm{N} 2$ & $118.72(16)$ \\
\hline $\mathrm{C} 14-\mathrm{C} 13-\mathrm{C} 12$ & $119.36(17)$ \\
\hline $\mathrm{C} 14-\mathrm{C} 13-\mathrm{H} 13 \mathrm{~A}$ & 120.3 \\
\hline $\mathrm{C} 12-\mathrm{C} 13-\mathrm{H} 13 \mathrm{~A}$ & 120.3 \\
\hline $\mathrm{C} 13-\mathrm{C} 14-\mathrm{C} 15$ & $119.85(18)$ \\
\hline $\mathrm{C} 13-\mathrm{C} 14-\mathrm{H} 14 \mathrm{~A}$ & 120.1 \\
\hline $\mathrm{C} 15-\mathrm{C} 14-\mathrm{H} 14 \mathrm{~A}$ & 120.1 \\
\hline $\mathrm{C} 16-\mathrm{C} 15-\mathrm{C} 14$ & $120.65(17)$ \\
\hline $\mathrm{C} 16-\mathrm{C} 15-\mathrm{S} 1$ & $119.58(14)$ \\
\hline $\mathrm{C} 1-\mathrm{N} 1-\mathrm{N} 2-\mathrm{C} 3$ & $0.4(2)$ \\
\hline $\mathrm{C} 1-\mathrm{N} 1-\mathrm{N} 2-\mathrm{C} 12$ & $-176.62(16)$ \\
\hline $\mathrm{N} 2-\mathrm{N} 1-\mathrm{C} 1-\mathrm{C} 2$ & $-0.2(2)$ \\
\hline $\mathrm{N} 2-\mathrm{N} 1-\mathrm{C} 1-\mathrm{C} 4$ & $-178.68(18)$ \\
\hline $\mathrm{N} 1-\mathrm{C} 1-\mathrm{C} 2-\mathrm{C} 3$ & $-0.1(2)$ \\
\hline $\mathrm{C} 4-\mathrm{C} 1-\mathrm{C} 2-\mathrm{C} 3$ & $178.2(2)$ \\
\hline $\mathrm{N} 1-\mathrm{N} 2-\mathrm{C} 3-\mathrm{C} 2$ & $-0.5(2)$ \\
\hline $\mathrm{C} 12-\mathrm{N} 2-\mathrm{C} 3-\mathrm{C} 2$ & $176.06(17)$ \\
\hline $\mathrm{N} 1-\mathrm{N} 2-\mathrm{C} 3-\mathrm{C} 5$ & $176.51(16)$ \\
\hline $\mathrm{C} 12-\mathrm{N} 2-\mathrm{C} 3-\mathrm{C} 5$ & $-6.9(3)$ \\
\hline $\mathrm{C} 1-\mathrm{C} 2-\mathrm{C} 3-\mathrm{N} 2$ & $0.4(2)$ \\
\hline
\end{tabular}

$\mathrm{C} 3 \mathrm{~S}-\mathrm{C} 4 \mathrm{~S}-\mathrm{H} 5 \mathrm{~S} \quad 109.3$

$\mathrm{N} 2 \mathrm{~S}-\mathrm{C} 4 \mathrm{~S}-\mathrm{H} 5 \mathrm{~S} \quad 109.3$

C3S-C4S-H6S 109.3

N2S-C4S-H6S $\quad 109.3$

H5S-C4S-H6S 108.0

C3SA-C2SA-N1S 118.4 (4)

C3SA-C2SA-H7S $\quad 107.7$

$\mathrm{N} 1 \mathrm{~S}-\mathrm{C} 2 \mathrm{SA}-\mathrm{H} 7 \mathrm{~S} \quad 107.7$

C3SA-C2SA-H8S $\quad 107.7$

N1S-C2SA-H8S 107.7

H7S-C2SA-H8S 107.1

C2SA-C3SA-C4SA 114.8 (6)

C2SA-C3SA-H9S $\quad 108.6$

C4SA-C3SA-H9S $\quad 108.6$

C2SA-C3SA-H10S $\quad 108.6$

C4SA-C3SA-H10S $\quad 108.6$

H9S-C3SA-H10S 107.5

N2S-C4SA-C3SA 116.8 (4)

N2S-C4SA-H11S $\quad 108.1$

C3SA-C4SA-H11S $\quad 108.1$

N2S-C4SA-H12S 108.1

C3SA-C4SA-H12S $\quad 108.1$

H11S-C4SA-H12S $\quad 107.3$

N1S-C5S-H13S $\quad 109.5$

N1S-C5S-H14S $\quad 109.5$

H13S-C5S-H14S 109.5

$\mathrm{N} 1 \mathrm{~S}-\mathrm{C} 5 \mathrm{~S}-\mathrm{H} 15 \mathrm{~S} \quad 109.5$

H13S-C5S-H15S 109.5

H14S-C5S-H15S 109.5

$\mathrm{N} 2 \mathrm{~S}-\mathrm{C} 6 \mathrm{~S}-\mathrm{H} 16 \mathrm{~S} \quad 109.5$

$\mathrm{N} 2 \mathrm{~S}-\mathrm{C} 6 \mathrm{~S}-\mathrm{H} 17 \mathrm{~S} \quad 109.5$

H16S-C6S-H17S $\quad 109.5$

$\mathrm{N} 2 \mathrm{~S}-\mathrm{C} 6 \mathrm{~S}-\mathrm{H} 18 \mathrm{~S} \quad 109.5$

H16S-C6S-H18S $\quad 109.5$

H17S-C6S-H18S $\quad 109.5$

$\begin{array}{ll}\mathrm{C} 3-\mathrm{N} 2-\mathrm{C} 12-\mathrm{C} 13 & 129.1(2) \\ \mathrm{C} 17-\mathrm{C} 12-\mathrm{C} 13-\mathrm{C} 14 & -0.5(3) \\ \mathrm{N} 2-\mathrm{C} 12-\mathrm{C} 13-\mathrm{C} 14 & 177.77(17) \\ \mathrm{C} 12-\mathrm{C} 13-\mathrm{C} 14-\mathrm{C} 15 & -0.2(3) \\ \mathrm{C} 13-\mathrm{C} 14-\mathrm{C} 15-\mathrm{C} 16 & 0.9(3) \\ \mathrm{C} 13-\mathrm{C} 14-\mathrm{C} 15-\mathrm{S} 1 & 179.45(15) \\ \mathrm{O} 1-\mathrm{S} 1-\mathrm{C} 15-\mathrm{C} 16 & -159.85(16) \\ \mathrm{O} 2-\mathrm{S} 1-\mathrm{C} 15-\mathrm{C} 16 & -29.88(18) \\ \mathrm{N} 3-\mathrm{S} 1-\mathrm{C} 15-\mathrm{C} 16 & 85.20(16) \\ \mathrm{O} 1-\mathrm{S} 1-\mathrm{C} 15-\mathrm{C} 14 & 21.56(19) \\ \mathrm{O} 2-\mathrm{S} 1-\mathrm{C} 15-\mathrm{C} 14 & 151.52(16)\end{array}$




$\begin{array}{ll}\mathrm{C} 1-\mathrm{C} 2-\mathrm{C} 3-\mathrm{C} 5 & -176.41(18) \\ \mathrm{N} 1-\mathrm{C} 1-\mathrm{C} 4-\mathrm{F} 3 \mathrm{~A} & -167.1(10) \\ \mathrm{C} 2-\mathrm{C} 1-\mathrm{C} 4-\mathrm{F} 3 \mathrm{~A} & 14.7(10) \\ \mathrm{N} 1-\mathrm{C} 1-\mathrm{C} 4-\mathrm{F} 2 \mathrm{~A} & 65.3(8) \\ \mathrm{C} 2-\mathrm{C} 1-\mathrm{C} 4-\mathrm{F} 2 \mathrm{~A} & -112.9(8) \\ \mathrm{N} 1-\mathrm{C} 1-\mathrm{C} 4-\mathrm{F} 1 & -81.5(10) \\ \mathrm{C} 2-\mathrm{C} 1-\mathrm{C} 4-\mathrm{F} 1 & 100.3(10) \\ \mathrm{N} 1-\mathrm{C} 1-\mathrm{C} 4-\mathrm{F} 2 & 45.9(6) \\ \mathrm{C} 2-\mathrm{C} 1-\mathrm{C} 4-\mathrm{F} 2 & -132.4(6) \\ \mathrm{N} 1-\mathrm{C} 1-\mathrm{C} 4-\mathrm{F} 3 & 160.7(6) \\ \mathrm{C} 2-\mathrm{C} 1-\mathrm{C} 4-\mathrm{F} 3 & -17.5(7) \\ \mathrm{N} 1-\mathrm{C} 1-\mathrm{C} 4-\mathrm{F} 1 \mathrm{~A} & -48.3(6) \\ \mathrm{C} 2-\mathrm{C} 1-\mathrm{C} 4-\mathrm{F} 1 \mathrm{~A} & 133.4(5) \\ \mathrm{N} 2-\mathrm{C} 3-\mathrm{C} 5-\mathrm{C} 10 & 144.19(18) \\ \mathrm{C} 2-\mathrm{C} 3-\mathrm{C} 5-\mathrm{C} 10 & -39.6(3) \\ \mathrm{N} 2-\mathrm{C} 3-\mathrm{C} 5-\mathrm{C} 6 & -39.0(3) \\ \mathrm{C} 2-\mathrm{C} 3-\mathrm{C} 5-\mathrm{C} 6 & 137.3(2) \\ \mathrm{C} 10-\mathrm{C} 5-\mathrm{C} 6-\mathrm{C} 7 & -0.7(3) \\ \mathrm{C} 3-\mathrm{C} 5-\mathrm{C} 6-\mathrm{C} 7 & -177.66(16) \\ \mathrm{C} 5-\mathrm{C} 6-\mathrm{C} 7-\mathrm{C} 8 & 0.1(3) \\ \mathrm{C} 6-\mathrm{C} 7-\mathrm{C} 8-\mathrm{C} 9 & 0.3(3) \\ \mathrm{C} 6-\mathrm{C} 7-\mathrm{C} 8-\mathrm{C} 11 & -179.29(18) \\ \mathrm{C} 7-\mathrm{C} 8-\mathrm{C} 9-\mathrm{C} 10 & -0.1(3) \\ \mathrm{C} 11-\mathrm{C} 8-\mathrm{C} 9-\mathrm{C} 10 & 179.5(2) \\ \mathrm{C} 8-\mathrm{C} 9-\mathrm{C} 10-\mathrm{C} 5 & -0.6(3) \\ \mathrm{C} 6-\mathrm{C} 5-\mathrm{C} 10-\mathrm{C} 9 & 1.0(3) \\ \mathrm{C} 3-\mathrm{C} 5-\mathrm{C} 10-\mathrm{C} 9 & 178.00(17) \\ \mathrm{N} 1-\mathrm{N} 2-\mathrm{C} 12-\mathrm{C} 17 & 123.84(19) \\ \mathrm{C} 3-\mathrm{N} 2-\mathrm{C} 12-\mathrm{C} 17 & -52.6(3) \\ \mathrm{N} 1-\mathrm{N} 2-\mathrm{C} 12-\mathrm{C} 13 & -54.4(2) \\ & \end{array}$

$\begin{array}{ll}\mathrm{N} 3-\mathrm{S} 1-\mathrm{C} 15-\mathrm{C} 14 & -93.40(17) \\ \mathrm{C} 14-\mathrm{C} 15-\mathrm{C} 16-\mathrm{C} 17 & -0.8(3) \\ \mathrm{S} 1-\mathrm{C} 15-\mathrm{C} 16-\mathrm{C} 17 & -179.37(14) \\ \mathrm{C} 13-\mathrm{C} 12-\mathrm{C} 17-\mathrm{C} 16 & 0.6(3) \\ \mathrm{N} 2-\mathrm{C} 12-\mathrm{C} 17-\mathrm{C} 16 & -177.67(16) \\ \mathrm{C} 15-\mathrm{C} 16-\mathrm{C} 17-\mathrm{C} 12 & 0.1(3) \\ \mathrm{C} 4 \mathrm{SA}-\mathrm{N} 2 \mathrm{~S}-\mathrm{C} 1 \mathrm{~S}-\mathrm{O} 1 \mathrm{~S} & 176.8(2) \\ \mathrm{C} 4 \mathrm{~S}-\mathrm{N} 2 \mathrm{~S}-\mathrm{C} 1 \mathrm{~S}-\mathrm{O} 1 \mathrm{~S} & 176.8(2) \\ \mathrm{C} 6 \mathrm{~S}-\mathrm{N} 2 \mathrm{~S}-\mathrm{C} 1 \mathrm{~S}-\mathrm{O} 1 \mathrm{~S} & 3.2(3) \\ \mathrm{C} 4 \mathrm{SA}-\mathrm{N} 2 \mathrm{~S}-\mathrm{C} 1 \mathrm{~S}-\mathrm{N} 1 \mathrm{~S} & -3.6(3) \\ \mathrm{C} 4 \mathrm{~S}-\mathrm{N} 2 \mathrm{~S}-\mathrm{C} 1 \mathrm{~S}-\mathrm{N} 1 \mathrm{~S} & -3.6(3) \\ \mathrm{C} 6 \mathrm{~S}-\mathrm{N} 2 \mathrm{~S}-\mathrm{C} 1 \mathrm{~S}-\mathrm{N} 1 \mathrm{~S} & -177.3(2) \\ \mathrm{C} 2 \mathrm{SA}-\mathrm{N} 1 \mathrm{~S}-\mathrm{C} 1 \mathrm{~S}-\mathrm{O} 1 \mathrm{~S} & -179.0(2) \\ \mathrm{C} 2 \mathrm{~S}-\mathrm{N} 1 \mathrm{~S}-\mathrm{C} 1 \mathrm{~S}-\mathrm{O} 1 \mathrm{~S} & -179.0(2) \\ \mathrm{C} 5 \mathrm{~S}-\mathrm{N} 1 \mathrm{~S}-\mathrm{C} 1 \mathrm{~S}-\mathrm{O} 1 \mathrm{~S} & -3.7(3) \\ \mathrm{C} 2 \mathrm{SA}-\mathrm{N} 1 \mathrm{~S}-\mathrm{C} 1 \mathrm{~S}-\mathrm{N} 2 \mathrm{~S} & 1.4(3) \\ \mathrm{C} 2 \mathrm{~S}-\mathrm{N} 1 \mathrm{~S}-\mathrm{C} 1 \mathrm{~S}-\mathrm{N} 2 \mathrm{~S} & 1.4(3) \\ \mathrm{C} 5 \mathrm{~S}-\mathrm{N} 1 \mathrm{~S}-\mathrm{C} 1 \mathrm{~S}-\mathrm{N} 2 \mathrm{~S} & 176.7(2) \\ \mathrm{C} 1 \mathrm{~S}-\mathrm{N} 1 \mathrm{~S}-\mathrm{C} 2 \mathrm{~S}-\mathrm{C} 3 \mathrm{~S} & -19.3(6) \\ \mathrm{C} 5 \mathrm{~S}-\mathrm{N} 1 \mathrm{~S}-\mathrm{C} 2 \mathrm{~S}-\mathrm{C} 3 \mathrm{~S} & 165.2(5) \\ \text { N1S-C2S-C3S-C4S } & 41.2(11) \\ \text { C2S-C3S-C4S-N2S } & -43.3(11) \\ \text { C1S-N2S-C4S-C3S } & 24.1(6) \\ \text { C6S-N2S-C4S-C3S } & -162.1(6) \\ \text { C1S-N1S-C2SA-C3SA } & 14.3(8) \\ \text { C5S-N1S-C2SA-C3SA } & -161.1(8) \\ \text { N1S-C2SA-C3SA-C4SA } & -25.6(13) \\ \text { C1S-N2S-C4SA-C3SA } & -8.9(8) \\ \text { C6S-N2S-C4SA-C3SA } & 164.9(7) \\ \text { C2SA-C3SA-C4SA-N2S } & 23.2(13) \\ & \end{array}$

Hydrogen-bond geometry $\left(A,{ }^{\circ}\right)$

\begin{tabular}{lllll}
\hline$D-\mathrm{H} \cdots A$ & $D-\mathrm{H}$ & $\mathrm{H} \cdots A$ & $D \cdots A$ & $D-\mathrm{H} \cdots A$ \\
\hline $\mathrm{N} 3-\mathrm{H} 1 N \cdots \mathrm{O} 1 S$ & $0.87(1)$ & $2.01(1)$ & $2.870(2)$ & $168(2)$ \\
$\mathrm{N} 3-\mathrm{H} 2 N \cdots \mathrm{O} 1 S^{\mathrm{i}}$ & $0.87(1)$ & $2.11(1)$ & $2.958(2)$ & $164(2)$ \\
\hline
\end{tabular}

Symmetry code: (i) $-x+1,-y+1,-z+1$.

4-[5-(4-Methylphenyl)-3-(trifluoromethyl)pyrazol-1-yl] benzenesulfonamide dimethyl sulfoxide monosolvate (6)

Crystal data

$\mathrm{C}_{17} \mathrm{H}_{14} \mathrm{~F}_{3} \mathrm{~N}_{3} \mathrm{O}_{2} \mathrm{~S} \cdot \mathrm{C}_{2} \mathrm{H}_{6} \mathrm{OS}$

$M_{r}=459.50$

Monoclinic, $P 2_{1} / c$

$a=11.9884(3) \AA$

$b=9.0230(3) \AA$

$c=20.8537(6) \AA$

$\beta=100.3908(9)^{\circ}$

$$
\begin{aligned}
& V=2218.78(11) \AA^{3} \\
& Z=4 \\
& F(000)=952 \\
& D_{\mathrm{x}}=1.376 \mathrm{Mg} \mathrm{m}^{-3}
\end{aligned}
$$

$\mathrm{Cu} K \alpha$ radiation, $\lambda=1.54178 \AA$

Cell parameters from 9824 reflections $\theta=4.0-66.8^{\circ}$ 
$\mu=2.63 \mathrm{~mm}^{-1}$

$T=298 \mathrm{~K}$

Data collection

Bruker D8-QUEST PHOTON-100 diffractometer

Radiation source: Incoatec $\mathrm{I} \mu \mathrm{S} \mathrm{Cu}$ microsource $\omega$ and $\varphi$-scans

Absorption correction: multi-scan

(SADABS; Bruker, 2016)

$T_{\min }=0.476, T_{\max }=0.753$

22464 measured reflections

Refinement

Refinement on $F^{2}$

Least-squares matrix: full

$R\left[F^{2}>2 \sigma\left(F^{2}\right)\right]=0.044$

$w R\left(F^{2}\right)=0.126$

$S=1.04$

3916 reflections

318 parameters

15 restraints

Primary atom site location: dual
Block, colourless

$0.14 \times 0.12 \times 0.12 \mathrm{~mm}$

3916 independent reflections

3520 reflections with $I>2 \sigma(I)$

$R_{\text {int }}=0.042$

$\theta_{\text {max }}=66.8^{\circ}, \theta_{\text {min }}=3.8^{\circ}$

$h=-14 \rightarrow 14$

$k=-10 \rightarrow 10$

$l=-24 \rightarrow 24$

Secondary atom site location: difference Fourier map

Hydrogen site location: mixed

$\mathrm{H}$ atoms treated by a mixture of independent and constrained refinement

$w=1 /\left[\sigma^{2}\left(F_{\mathrm{o}}^{2}\right)+(0.065 P)^{2}+0.7974 P\right]$ where $P=\left(F_{\mathrm{o}}^{2}+2 F_{\mathrm{c}}^{2}\right) / 3$

$(\Delta / \sigma)_{\max }=0.002$

$\Delta \rho_{\max }=0.29 \mathrm{e}^{-3}$

$\Delta \rho_{\min }=-0.28$ e $\AA^{-3}$

\section{Special details}

Geometry. All esds (except the esd in the dihedral angle between two 1.s. planes) are estimated using the full covariance matrix. The cell esds are taken into account individually in the estimation of esds in distances, angles and torsion angles; correlations between esds in cell parameters are only used when they are defined by crystal symmetry. An approximate (isotropic) treatment of cell esds is used for estimating esds involving l.s. planes.

Fractional atomic coordinates and isotropic or equivalent isotropic displacement parameters $\left(\AA^{2}\right)$

\begin{tabular}{llllll}
\hline & $x$ & $y$ & $z$ & $U_{\text {iso }}^{*} / U_{\text {eq }}$ & Occ. $(<1)$ \\
\hline S1 & $0.44222(4)$ & $0.73094(6)$ & $0.58322(3)$ & $0.05825(19)$ & \\
O1 & $0.43028(13)$ & $0.6822(2)$ & $0.64670(8)$ & $0.0748(5)$ & \\
O2 & $0.38751(13)$ & $0.8632(2)$ & $0.55698(10)$ & $0.0865(6)$ & \\
$\mathrm{N} 1$ & $0.99161(13)$ & $0.8825(2)$ & $0.64689(8)$ & $0.0614(4)$ & \\
$\mathrm{N} 2$ & $0.93516(12)$ & $0.8229(2)$ & $0.59031(8)$ & $0.0520(4)$ & \\
$\mathrm{N} 3$ & $0.39656(15)$ & $0.5997(3)$ & $0.53365(10)$ & $0.0695(5)$ & \\
$\mathrm{H} 1 \mathrm{~N}$ & $0.403(2)$ & $0.621(3)$ & $0.4937(7)$ & $0.086(8)^{*}$ & \\
$\mathrm{H} 2 \mathrm{~N}$ & $0.426(2)$ & $0.5160(18)$ & $0.5493(10)$ & $0.080(8)^{*}$ & \\
$\mathrm{C} 1$ & $1.09613(16)$ & $0.8974(3)$ & $0.63600(11)$ & $0.0616(5)$ & \\
$\mathrm{C} 2$ & $1.10903(15)$ & $0.8487(2)$ & $0.57482(11)$ & $0.0584(5)$ & \\
$\mathrm{H} 2 \mathrm{~A}$ & 1.175041 & 0.849422 & 0.557261 & $0.070^{*}$ & \\
$\mathrm{C} 3$ & $1.00368(14)$ & $0.7992(2)$ & $0.54545(9)$ & $0.0501(4)$ & \\
$\mathrm{C} 4$ & $1.1841(2)$ & $0.9581(3)$ & $0.68942(14)$ & $0.0872(8)$ & \\
F1 & $1.2791(4)$ & $0.8978(10)$ & $0.6941(3)$ & $0.103(3)$ & $0.435(10)$ \\
F2 & $1.2022(10)$ & $1.0988(6)$ & $0.6870(8)$ & $0.217(8)$ & $0.435(10)$ \\
F3 & $1.1604(5)$ & $0.9368(15)$ & $0.7500(3)$ & $0.128(5)$ & $0.435(10)$ \\
F1A & $1.2289(11)$ & $0.8568(7)$ & $0.7273(6)$ & $0.244(8)$ & $0.565(10)$ \\
F2A & $1.2654(7)$ & $1.0176(14)$ & $0.6642(4)$ & $0.198(5)$ & $0.565(10)$
\end{tabular}




\begin{tabular}{|c|c|c|c|c|c|}
\hline F3A & $1.1478(4)$ & $1.0607(9)$ & $0.7190(3)$ & $0.122(3)$ & $0.565(10)$ \\
\hline $\mathrm{C} 5$ & $0.96935(15)$ & $0.7264(2)$ & $0.48219(9)$ & $0.0504(4)$ & \\
\hline C6 & $0.88711(16)$ & $0.6159(2)$ & $0.47095(10)$ & $0.0550(5)$ & \\
\hline H6A & 0.849346 & 0.587856 & 0.504251 & $0.066^{*}$ & \\
\hline $\mathrm{C} 7$ & $0.86100(17)$ & $0.5475(2)$ & $0.41090(11)$ & $0.0618(5)$ & \\
\hline $\mathrm{H} 7 \mathrm{~A}$ & 0.804298 & 0.475879 & 0.404152 & $0.074 *$ & \\
\hline $\mathrm{C} 8$ & $0.9169(2)$ & $0.5827(3)$ & $0.36076(11)$ & $0.0689(6)$ & \\
\hline C9 & $1.0000(2)$ & $0.6903(3)$ & $0.37240(12)$ & $0.0794(7)$ & \\
\hline H9A & 1.039563 & 0.715210 & 0.339438 & $0.095^{*}$ & \\
\hline $\mathrm{C} 10$ & $1.0259(2)$ & $0.7618(3)$ & $0.43164(11)$ & $0.0686(6)$ & \\
\hline $\mathrm{H} 10 \mathrm{~A}$ & 1.081833 & 0.834444 & 0.437849 & $0.082^{*}$ & \\
\hline $\mathrm{C} 11$ & $0.8873(3)$ & $0.5063(4)$ & $0.29538(13)$ & $0.1020(9)$ & \\
\hline H11A & 0.827940 & 0.435486 & 0.296642 & $0.153^{*}$ & \\
\hline H11B & 0.861991 & 0.578628 & 0.262165 & $0.153^{*}$ & \\
\hline $\mathrm{H} 11 \mathrm{C}$ & 0.953037 & 0.456362 & 0.285822 & $0.153^{*}$ & \\
\hline $\mathrm{C} 12$ & $0.81593(14)$ & $0.7978(2)$ & $0.58644(9)$ & $0.0479(4)$ & \\
\hline $\mathrm{C} 13$ & $0.66715(16)$ & $0.6961(3)$ & $0.63440(10)$ & $0.0607(5)$ & \\
\hline $\mathrm{H} 13 \mathrm{~A}$ & 0.642776 & 0.642659 & 0.667416 & $0.073^{*}$ & \\
\hline $\mathrm{C} 14$ & $0.78154(16)$ & $0.7171(3)$ & $0.63526(10)$ & $0.0618(5)$ & \\
\hline H14A & 0.834895 & 0.676792 & 0.668636 & $0.074 *$ & \\
\hline C15 & $0.58944(14)$ & $0.7553(2)$ & $0.58406(9)$ & $0.0482(4)$ & \\
\hline $\mathrm{C} 16$ & $0.62439(15)$ & $0.8371(2)$ & $0.53513(9)$ & $0.0518(5)$ & \\
\hline H16A & 0.571201 & 0.877375 & 0.501672 & $0.062^{*}$ & \\
\hline $\mathrm{C} 17$ & $0.73877(15)$ & $0.8585(2)$ & $0.53635(9)$ & $0.0519(5)$ & \\
\hline H17A & 0.763427 & 0.913147 & 0.503771 & $0.062^{*}$ & \\
\hline O1S & $0.47134(17)$ & $0.6580(2)$ & $0.41112(8)$ & $0.0915(6)$ & \\
\hline S1S & $0.43698(8)$ & $0.68487(10)$ & $0.34114(4)$ & $0.0876(4)$ & $0.807(3)$ \\
\hline $\mathrm{C} 1 \mathrm{~S}$ & $0.5584(5)$ & $0.6975(8)$ & $0.3078(2)$ & $0.198(3)$ & $0.807(3)$ \\
\hline H1S1 & 0.538075 & 0.715194 & 0.261751 & $0.298^{*}$ & $0.807(3)$ \\
\hline H1S2 & 0.604363 & 0.777903 & 0.327870 & $0.298^{*}$ & $0.807(3)$ \\
\hline $\mathrm{H} 1 \mathrm{~S} 3$ & 0.600290 & 0.606580 & 0.315160 & $0.298^{*}$ & $0.807(3)$ \\
\hline $\mathrm{C} 2 \mathrm{~S}$ & $0.3926(7)$ & $0.8682(6)$ & $0.3303(3)$ & $0.235(4)$ & 0.807 (3) \\
\hline $\mathrm{H} 2 \mathrm{~S} 1$ & 0.369592 & 0.888148 & 0.284653 & $0.352^{*}$ & $0.807(3)$ \\
\hline $\mathrm{H} 2 \mathrm{~S} 2$ & 0.329712 & 0.884567 & 0.352215 & $0.352^{*}$ & $0.807(3)$ \\
\hline $\mathrm{H} 2 \mathrm{~S} 3$ & 0.453870 & 0.932914 & 0.348210 & $0.352^{*}$ & $0.807(3)$ \\
\hline S1SA & $0.5073(7)$ & $0.7789(6)$ & 0.3699 (2) & $0.153(3)$ & 0.193 \\
\hline C1SA & $0.5584(5)$ & $0.6975(8)$ & $0.3078(2)$ & $0.198(3)$ & $0.193(3)$ \\
\hline H1S4 & 0.582101 & 0.772639 & 0.280502 & $0.298^{*}$ & $0.193(3)$ \\
\hline H1S5 & 0.621958 & 0.635925 & 0.325183 & $0.298^{*}$ & $0.193(3)$ \\
\hline H1S6 & 0.500104 & 0.638050 & 0.282502 & $0.298^{*}$ & $0.193(3)$ \\
\hline $\mathrm{C} 2 \mathrm{SA}$ & $0.3926(7)$ & $0.8682(6)$ & $0.3303(3)$ & $0.235(4)$ & $0.193(3)$ \\
\hline $\mathrm{H} 2 \mathrm{~S} 4$ & 0.415844 & 0.945446 & 0.303989 & $0.352^{*}$ & $0.193(3)$ \\
\hline $\mathrm{H} 2 \mathrm{~S} 5$ & 0.344523 & 0.799568 & 0.302991 & $0.352^{*}$ & $0.193(3)$ \\
\hline H2S6 & 0.351645 & 0.910595 & 0.361412 & $0.352^{*}$ & $0.193(3)$ \\
\hline
\end{tabular}


Atomic displacement parameters $\left(\AA^{2}\right)$

\begin{tabular}{|c|c|c|c|c|c|c|}
\hline & $U^{11}$ & $U^{22}$ & $U^{33}$ & $U^{12}$ & $U^{13}$ & $U^{23}$ \\
\hline $\mathrm{S} 1$ & $0.0349(3)$ & 0.0718 & $0.0718(3)$ & $0.0014(2)$ & $0.0198(2)$ & $0.0103(2)$ \\
\hline $\mathrm{O} 1$ & $0.0589(9)$ & $0.1013(12)$ & $0.0720(10)$ & $-0.0075(8)$ & $0.0328(7)$ & $0.0038(9)$ \\
\hline $\mathrm{O} 2$ & $0.0450(8)$ & $0.0863(12)$ & $0.1343(15)$ & $0.0178(8)$ & $0.0321(9)$ & $0.0303(11)$ \\
\hline N1 & $0.0415(8)$ & $0.0819(12)$ & $0.0585(9)$ & $-0.0047(8)$ & $0.0026(7)$ & $-0.0073(9)$ \\
\hline N2 & $0.0328(7)$ & $0.0692(10)$ & $0.0534(9)$ & $-0.0038(7)$ & $0.0056(6)$ & $0.0012(7)$ \\
\hline N3 & $0.0484(10)$ & $0.0910(15)$ & $0.0675(12)$ & $-0.0139(10)$ & $0.0058(8)$ & $0.0101(11)$ \\
\hline $\mathrm{C} 1$ & $0.0364(9)$ & $0.0714(13)$ & $0.0740(13)$ & $-0.0043(9)$ & $0.0018(9)$ & $-0.0072(10)$ \\
\hline $\mathrm{C} 2$ & $0.0331(9)$ & $0.0646(12)$ & $0.0778(13)$ & $-0.0026(8)$ & $0.0111(8)$ & $-0.0024(10)$ \\
\hline $\mathrm{C} 3$ & $0.0348(9)$ & $0.0554(11)$ & $0.0603(11)$ & $-0.0001(8)$ & $0.0090(8)$ & $0.0042(8)$ \\
\hline $\mathrm{C} 4$ & $0.0521(14)$ & $0.105(2)$ & 0.0974 (19) & $-0.0078(14)$ & $-0.0043(13)$ & $-0.0276(18)$ \\
\hline $\mathrm{F} 1$ & $0.040(2)$ & $0.168(7)$ & $0.091(3)$ & $0.032(3)$ & $-0.0195(19)$ & -0.049 (4) \\
\hline $\mathrm{F} 2$ & $0.174(11)$ & $0.084(4)$ & $0.322(16)$ & $-0.047(6)$ & $-0.146(10)$ & $0.020(6)$ \\
\hline F3 & $0.078(3)$ & 0.247 (12) & $0.060(3)$ & $-0.047(5)$ & $0.013(3)$ & $-0.044(5)$ \\
\hline F1A & $0.220(11)$ & $0.175(6)$ & $0.252(12)$ & $-0.035(7)$ & $-0.183(10)$ & $0.043(7)$ \\
\hline F2A & $0.104(4)$ & $0.299(12)$ & $0.207(7)$ & $-0.129(6)$ & $0.070(5)$ & $-0.158(8)$ \\
\hline F3A & $0.093(3)$ & $0.172(7)$ & $0.088(3)$ & 0.009 (3) & $-0.016(2)$ & $-0.072(4)$ \\
\hline $\mathrm{C} 5$ & $0.0370(9)$ & $0.0566(11)$ & $0.0585(11)$ & $0.0029(8)$ & $0.0107(8)$ & $0.0044(8)$ \\
\hline C6 & $0.0441(10)$ & $0.0598(11)$ & $0.0622(11)$ & $-0.0024(9)$ & $0.0126(8)$ & $0.0030(9)$ \\
\hline $\mathrm{C} 7$ & $0.0505(11)$ & $0.0622(12)$ & 0.0717 (13) & $-0.0031(9)$ & $0.0084(9)$ & $-0.0029(10)$ \\
\hline $\mathrm{C} 8$ & $0.0681(13)$ & $0.0755(15)$ & $0.0628(12)$ & $0.0020(11)$ & $0.0108(10)$ & $-0.0029(11)$ \\
\hline $\mathrm{C} 9$ & $0.0817(16)$ & 0.0945 (18) & $0.0695(14)$ & $-0.0117(14)$ & $0.0335(12)$ & $-0.0008(13)$ \\
\hline $\mathrm{C} 10$ & 0.0602 (13) & 0.0785 (15) & $0.0713(14)$ & $-0.0154(11)$ & $0.0231(11)$ & $-0.0008(11)$ \\
\hline $\mathrm{C} 11$ & $0.118(2)$ & $0.115(2)$ & $0.0732(16)$ & $-0.010(2)$ & $0.0190(15)$ & $-0.0184(16)$ \\
\hline $\mathrm{C} 12$ & $0.0321(8)$ & $0.0598(11)$ & $0.0517(10)$ & $-0.0025(8)$ & $0.0077(7)$ & $0.0012(8)$ \\
\hline $\mathrm{C} 13$ & $0.0450(10)$ & $0.0801(14)$ & $0.0584(11)$ & $-0.0032(10)$ & $0.0134(8)$ & $0.0204(10)$ \\
\hline C14 & $0.0413(10)$ & $0.0853(15)$ & $0.0570(11)$ & $0.0001(10)$ & $0.0040(8)$ & $0.0206(10)$ \\
\hline C15 & $0.0343(9)$ & $0.0565(10)$ & $0.0553(10)$ & $-0.0003(8)$ & $0.0125(7)$ & $0.0025(8)$ \\
\hline C16 & $0.0359(9)$ & $0.0637(12)$ & $0.0556(10)$ & $0.0034(8)$ & $0.0072(7)$ & $0.0121(9)$ \\
\hline C17 & $0.0401(9)$ & $0.0631(12)$ & $0.0537(10)$ & $-0.0017(8)$ & $0.0119(8)$ & $0.0124(9)$ \\
\hline O1S & 0.1077 (14) & $0.1002(14)$ & $0.0645(10)$ & $-0.0028(11)$ & $0.0097(9)$ & $0.0122(9)$ \\
\hline S1S & $0.1060(7)$ & $0.0805(6)$ & $0.0628(5)$ & $-0.0200(5)$ & $-0.0212(4)$ & $0.0063(4)$ \\
\hline C1S & $0.224(6)$ & $0.257(7)$ & $0.130(4)$ & $-0.029(5)$ & $0.072(4)$ & $0.069(4)$ \\
\hline $\mathrm{C} 2 \mathrm{~S}$ & $0.390(10)$ & $0.126(4)$ & $0.150(4)$ & $0.094(5)$ & $-0.056(5)$ & $0.011(3)$ \\
\hline S1SA & $0.249(8)$ & $0.106(3)$ & $0.074(3)$ & $-0.082(4)$ & $-0.046(3)$ & $0.016(2)$ \\
\hline C1SA & $0.224(6)$ & $0.257(7)$ & $0.130(4)$ & $-0.029(5)$ & $0.072(4)$ & $0.069(4)$ \\
\hline C2SA & $0.390(10)$ & $0.126(4)$ & $0.150(4)$ & $0.094(5)$ & $-0.056(5)$ & $0.011(3)$ \\
\hline
\end{tabular}

Geometric parameters $\left(\AA,{ }^{\circ}\right)$

\begin{tabular}{lllr}
\hline $\mathrm{S} 1-\mathrm{O} 2$ & $1.4227(17)$ & $\mathrm{C} 11-\mathrm{H} 11 \mathrm{~A}$ & 0.9600 \\
$\mathrm{~S} 1-\mathrm{O} 1$ & $1.4265(16)$ & $\mathrm{C} 11-\mathrm{H} 11 \mathrm{~B}$ & 0.9600 \\
$\mathrm{~S} 1-\mathrm{N} 3$ & $1.602(2)$ & $\mathrm{C} 11-\mathrm{H} 11 \mathrm{C}$ & 0.9600 \\
$\mathrm{~S} 1-\mathrm{C} 15$ & $1.7755(17)$ & $\mathrm{C} 12-\mathrm{C} 14$ & $1.375(3)$ \\
$\mathrm{N} 1-\mathrm{C} 1$ & $\mathrm{C} 12-\mathrm{C} 17$ & $1.378(3)$ \\
$\mathrm{N} 1-\mathrm{N} 2$ & $1.320(3)$ & $\mathrm{C} 13-\mathrm{C} 15$ & $1.379(3)$
\end{tabular}




\begin{tabular}{|c|c|c|c|}
\hline $\mathrm{N} 2-\mathrm{C} 3$ & $1.369(2)$ & $\mathrm{C} 13-\mathrm{C} 14$ & $1.381(3)$ \\
\hline $\mathrm{N} 2-\mathrm{C} 12$ & $1.435(2)$ & $\mathrm{C} 13-\mathrm{H} 13 \mathrm{~A}$ & 0.9300 \\
\hline $\mathrm{N} 3-\mathrm{H} 1 \mathrm{~N}$ & $0.873(9)$ & $\mathrm{C} 14-\mathrm{H} 14 \mathrm{~A}$ & 0.9300 \\
\hline $\mathrm{N} 3-\mathrm{H} 2 \mathrm{~N}$ & $0.870(9)$ & $\mathrm{C} 15-\mathrm{C} 16$ & $1.384(3)$ \\
\hline $\mathrm{C} 1-\mathrm{C} 2$ & $1.384(3)$ & $\mathrm{C} 16-\mathrm{C} 17$ & $1.380(2)$ \\
\hline $\mathrm{C} 1-\mathrm{C} 4$ & $1.493(3)$ & $\mathrm{C} 16-\mathrm{H} 16 \mathrm{~A}$ & 0.9300 \\
\hline $\mathrm{C} 2-\mathrm{C} 3$ & $1.375(3)$ & C17-H17A & 0.9300 \\
\hline $\mathrm{C} 2-\mathrm{H} 2 \mathrm{~A}$ & 0.9300 & $\mathrm{O} 1 \mathrm{~S}-\mathrm{S} 1 \mathrm{~S}$ & $1.4634(18)$ \\
\hline $\mathrm{C} 3-\mathrm{C} 5$ & $1.464(3)$ & O1S-S1SA & $1.499(5)$ \\
\hline $\mathrm{C} 4-\mathrm{F} 3 \mathrm{~A}$ & $1.234(4)$ & $\mathrm{S} 1 \mathrm{~S}-\mathrm{C} 1 \mathrm{~S}$ & $1.727(5)$ \\
\hline $\mathrm{C} 4-\mathrm{F} 1$ & $1.250(5)$ & $\mathrm{S} 1 \mathrm{~S}-\mathrm{C} 2 \mathrm{~S}$ & $1.740(5)$ \\
\hline $\mathrm{C} 4-\mathrm{F} 1 \mathrm{~A}$ & $1.263(5)$ & $\mathrm{C} 1 \mathrm{~S}-\mathrm{H} 1 \mathrm{~S} 1$ & 0.9600 \\
\hline $\mathrm{C} 4-\mathrm{F} 2$ & $1.291(5)$ & $\mathrm{C} 1 \mathrm{~S}-\mathrm{H} 1 \mathrm{~S} 2$ & 0.9600 \\
\hline $\mathrm{C} 4-\mathrm{F} 2 \mathrm{~A}$ & $1.303(6)$ & $\mathrm{C} 1 \mathrm{~S}-\mathrm{H} 1 \mathrm{~S} 3$ & 0.9600 \\
\hline $\mathrm{C} 4-\mathrm{F} 3$ & $1.358(6)$ & $\mathrm{C} 2 \mathrm{~S}-\mathrm{H} 2 \mathrm{~S} 1$ & 0.9600 \\
\hline $\mathrm{C} 5-\mathrm{C} 10$ & $1.389(3)$ & $\mathrm{C} 2 \mathrm{~S}-\mathrm{H} 2 \mathrm{~S} 2$ & 0.9600 \\
\hline $\mathrm{C} 5-\mathrm{C} 6$ & $1.392(3)$ & $\mathrm{C} 2 \mathrm{~S}-\mathrm{H} 2 \mathrm{~S} 3$ & 0.9600 \\
\hline $\mathrm{C} 6-\mathrm{C} 7$ & $1.380(3)$ & $\mathrm{S} 1 \mathrm{SA}-\mathrm{C} 2 \mathrm{SA}$ & $1.676(9)$ \\
\hline C6-H6A & 0.9300 & $\mathrm{~S} 1 \mathrm{SA}-\mathrm{C} 1 \mathrm{SA}$ & $1.697(9)$ \\
\hline $\mathrm{C} 7-\mathrm{C} 8$ & $1.376(3)$ & $\mathrm{C} 1 \mathrm{SA}-\mathrm{H} 1 \mathrm{~S} 4$ & 0.9600 \\
\hline C7-H7A & 0.9300 & $\mathrm{C} 1 \mathrm{SA}-\mathrm{H} 1 \mathrm{~S} 5$ & 0.9600 \\
\hline $\mathrm{C} 8-\mathrm{C} 9$ & $1.382(4)$ & $\mathrm{C} 1 \mathrm{SA}-\mathrm{H} 1 \mathrm{~S} 6$ & 0.9600 \\
\hline $\mathrm{C} 8-\mathrm{C} 11$ & $1.512(3)$ & $\mathrm{C} 2 \mathrm{SA}-\mathrm{H} 2 \mathrm{~S} 4$ & 0.9600 \\
\hline $\mathrm{C} 9-\mathrm{C} 10$ & $1.378(3)$ & $\mathrm{C} 2 \mathrm{SA}-\mathrm{H} 2 \mathrm{~S} 5$ & 0.9600 \\
\hline C9-H9A & 0.9300 & $\mathrm{C} 2 \mathrm{SA}-\mathrm{H} 2 \mathrm{~S} 6$ & 0.9600 \\
\hline $\mathrm{C} 10-\mathrm{H} 10 \mathrm{~A}$ & 0.9300 & & \\
\hline $\mathrm{O} 2-\mathrm{S} 1-\mathrm{O} 1$ & $119.93(11)$ & $\mathrm{C} 8-\mathrm{C} 11-\mathrm{H} 11 \mathrm{~B}$ & 109.5 \\
\hline $\mathrm{O} 2-\mathrm{S} 1-\mathrm{N} 3$ & $106.97(12)$ & $\mathrm{H} 11 \mathrm{~A}-\mathrm{C} 11-\mathrm{H} 11 \mathrm{~B}$ & 109.5 \\
\hline $\mathrm{O} 1-\mathrm{S} 1-\mathrm{N} 3$ & $106.92(11)$ & $\mathrm{C} 8-\mathrm{C} 11-\mathrm{H} 11 \mathrm{C}$ & 109.5 \\
\hline $\mathrm{O} 2-\mathrm{S} 1-\mathrm{C} 15$ & $106.76(9)$ & $\mathrm{H} 11 \mathrm{~A}-\mathrm{C} 11-\mathrm{H} 11 \mathrm{C}$ & 109.5 \\
\hline $\mathrm{O} 1-\mathrm{S} 1-\mathrm{C} 15$ & $107.15(9)$ & $\mathrm{H} 11 \mathrm{~B}-\mathrm{C} 11-\mathrm{H} 11 \mathrm{C}$ & 109.5 \\
\hline $\mathrm{N} 3-\mathrm{S} 1-\mathrm{C} 15$ & $108.74(9)$ & $\mathrm{C} 14-\mathrm{C} 12-\mathrm{C} 17$ & $121.48(16)$ \\
\hline $\mathrm{C} 1-\mathrm{N} 1-\mathrm{N} 2$ & $103.30(16)$ & $\mathrm{C} 14-\mathrm{C} 12-\mathrm{N} 2$ & $118.09(16)$ \\
\hline $\mathrm{N} 1-\mathrm{N} 2-\mathrm{C} 3$ & $112.88(14)$ & $\mathrm{C} 17-\mathrm{C} 12-\mathrm{N} 2$ & $120.38(16)$ \\
\hline $\mathrm{N} 1-\mathrm{N} 2-\mathrm{C} 12$ & $116.47(15)$ & $\mathrm{C} 15-\mathrm{C} 13-\mathrm{C} 14$ & $119.33(18)$ \\
\hline $\mathrm{C} 3-\mathrm{N} 2-\mathrm{C} 12$ & $130.64(16)$ & $\mathrm{C} 15-\mathrm{C} 13-\mathrm{H} 13 \mathrm{~A}$ & 120.3 \\
\hline $\mathrm{S} 1-\mathrm{N} 3-\mathrm{H} 1 \mathrm{~N}$ & $111.8(18)$ & $\mathrm{C} 14-\mathrm{C} 13-\mathrm{H} 13 \mathrm{~A}$ & 120.3 \\
\hline $\mathrm{S} 1-\mathrm{N} 3-\mathrm{H} 2 \mathrm{~N}$ & $109.5(17)$ & $\mathrm{C} 12-\mathrm{C} 14-\mathrm{C} 13$ & $119.51(18)$ \\
\hline $\mathrm{H} 1 \mathrm{~N}-\mathrm{N} 3-\mathrm{H} 2 \mathrm{~N}$ & $116.7(16)$ & $\mathrm{C} 12-\mathrm{C} 14-\mathrm{H} 14 \mathrm{~A}$ & 120.2 \\
\hline $\mathrm{N} 1-\mathrm{C} 1-\mathrm{C} 2$ & $113.18(17)$ & $\mathrm{C} 13-\mathrm{C} 14-\mathrm{H} 14 \mathrm{~A}$ & 120.2 \\
\hline $\mathrm{N} 1-\mathrm{C} 1-\mathrm{C} 4$ & $118.3(2)$ & $\mathrm{C} 13-\mathrm{C} 15-\mathrm{C} 16$ & $120.99(16)$ \\
\hline $\mathrm{C} 2-\mathrm{C} 1-\mathrm{C} 4$ & $128.51(19)$ & $\mathrm{C} 13-\mathrm{C} 15-\mathrm{S} 1$ & $119.56(14)$ \\
\hline $\mathrm{C} 3-\mathrm{C} 2-\mathrm{C} 1$ & $105.51(17)$ & $\mathrm{C} 16-\mathrm{C} 15-\mathrm{S} 1$ & $119.43(14)$ \\
\hline $\mathrm{C} 3-\mathrm{C} 2-\mathrm{H} 2 \mathrm{~A}$ & 127.2 & $\mathrm{C} 17-\mathrm{C} 16-\mathrm{C} 15$ & $119.51(17)$ \\
\hline $\mathrm{C} 1-\mathrm{C} 2-\mathrm{H} 2 \mathrm{~A}$ & 127.2 & $\mathrm{C} 17-\mathrm{C} 16-\mathrm{H} 16 \mathrm{~A}$ & 120.2 \\
\hline $\mathrm{N} 2-\mathrm{C} 3-\mathrm{C} 2$ & $105.12(17)$ & $\mathrm{C} 15-\mathrm{C} 16-\mathrm{H} 16 \mathrm{~A}$ & 120.2 \\
\hline $\mathrm{N} 2-\mathrm{C} 3-\mathrm{C} 5$ & $125.46(16)$ & $\mathrm{C} 12-\mathrm{C} 17-\mathrm{C} 16$ & $119.18(17)$ \\
\hline
\end{tabular}




\begin{tabular}{|c|c|}
\hline $\mathrm{C} 2-\mathrm{C} 3-\mathrm{C} 5$ & $129.29(18)$ \\
\hline $\mathrm{F} 3 \mathrm{~A}-\mathrm{C} 4-\mathrm{F} 1 \mathrm{~A}$ & $112.3(6)$ \\
\hline $\mathrm{F} 1-\mathrm{C} 4-\mathrm{F} 2$ & $105.9(5)$ \\
\hline $\mathrm{F} 3 \mathrm{~A}-\mathrm{C} 4-\mathrm{F} 2 \mathrm{~A}$ & $104.4(4)$ \\
\hline $\mathrm{F} 1 \mathrm{~A}-\mathrm{C} 4-\mathrm{F} 2 \mathrm{~A}$ & $106.6(5)$ \\
\hline $\mathrm{F} 1-\mathrm{C} 4-\mathrm{F} 3$ & $101.9(5)$ \\
\hline $\mathrm{F} 2-\mathrm{C} 4-\mathrm{F} 3$ & $103.9(6)$ \\
\hline $\mathrm{F} 3 \mathrm{~A}-\mathrm{C} 4-\mathrm{C} 1$ & $112.5(3)$ \\
\hline $\mathrm{F} 1-\mathrm{C} 4-\mathrm{C} 1$ & $114.2(3)$ \\
\hline $\mathrm{F} 1 \mathrm{~A}-\mathrm{C} 4-\mathrm{C} 1$ & $111.4(3)$ \\
\hline $\mathrm{F} 2-\mathrm{C} 4-\mathrm{C} 1$ & $115.7(4)$ \\
\hline $\mathrm{F} 2 \mathrm{~A}-\mathrm{C} 4-\mathrm{C} 1$ & $109.2(4)$ \\
\hline $\mathrm{F} 3-\mathrm{C} 4-\mathrm{C} 1$ & $113.8(3)$ \\
\hline $\mathrm{C} 10-\mathrm{C} 5-\mathrm{C} 6$ & $117.66(19)$ \\
\hline $\mathrm{C} 10-\mathrm{C} 5-\mathrm{C} 3$ & $118.96(18)$ \\
\hline $\mathrm{C} 6-\mathrm{C} 5-\mathrm{C} 3$ & $123.25(17)$ \\
\hline $\mathrm{C} 7-\mathrm{C} 6-\mathrm{C} 5$ & $120.73(19)$ \\
\hline $\mathrm{C} 7-\mathrm{C} 6-\mathrm{H} 6 \mathrm{~A}$ & 119.6 \\
\hline $\mathrm{C} 5-\mathrm{C} 6-\mathrm{H} 6 \mathrm{~A}$ & 119.6 \\
\hline $\mathrm{C} 6-\mathrm{C} 7-\mathrm{C} 8$ & $121.6(2)$ \\
\hline $\mathrm{C} 6-\mathrm{C} 7-\mathrm{H} 7 \mathrm{~A}$ & 119.2 \\
\hline $\mathrm{C} 8-\mathrm{C} 7-\mathrm{H} 7 \mathrm{~A}$ & 119.2 \\
\hline $\mathrm{C} 9-\mathrm{C} 8-\mathrm{C} 7$ & $117.6(2)$ \\
\hline $\mathrm{C} 9-\mathrm{C} 8-\mathrm{C} 11$ & $121.6(2)$ \\
\hline $\mathrm{C} 7-\mathrm{C} 8-\mathrm{C} 11$ & $120.8(2)$ \\
\hline $\mathrm{C} 10-\mathrm{C} 9-\mathrm{C} 8$ & $121.7(2)$ \\
\hline $\mathrm{C} 10-\mathrm{C} 9-\mathrm{H} 9 \mathrm{~A}$ & 119.2 \\
\hline $\mathrm{C} 8-\mathrm{C} 9-\mathrm{H} 9 \mathrm{~A}$ & 119.2 \\
\hline $\mathrm{C} 9-\mathrm{C} 10-\mathrm{C} 5$ & $120.7(2)$ \\
\hline $\mathrm{C} 9-\mathrm{C} 10-\mathrm{H} 10 \mathrm{~A}$ & 119.6 \\
\hline $\mathrm{C} 5-\mathrm{C} 10-\mathrm{H} 10 \mathrm{~A}$ & 119.6 \\
\hline $\mathrm{C} 8-\mathrm{C} 11-\mathrm{H} 11 \mathrm{~A}$ & 109.5 \\
\hline $\mathrm{C} 1-\mathrm{N} 1-\mathrm{N} 2-\mathrm{C} 3$ & $0.9(2)$ \\
\hline $\mathrm{C} 1-\mathrm{N} 1-\mathrm{N} 2-\mathrm{C} 12$ & $-178.05(18)$ \\
\hline $\mathrm{N} 2-\mathrm{N} 1-\mathrm{C} 1-\mathrm{C} 2$ & $-0.4(3)$ \\
\hline $\mathrm{N} 2-\mathrm{N} 1-\mathrm{C} 1-\mathrm{C} 4$ & $-178.7(2)$ \\
\hline $\mathrm{N} 1-\mathrm{C} 1-\mathrm{C} 2-\mathrm{C} 3$ & $-0.2(3)$ \\
\hline $\mathrm{C} 4-\mathrm{C} 1-\mathrm{C} 2-\mathrm{C} 3$ & $177.9(2)$ \\
\hline $\mathrm{N} 1-\mathrm{N} 2-\mathrm{C} 3-\mathrm{C} 2$ & $-1.0(2)$ \\
\hline $\mathrm{C} 12-\mathrm{N} 2-\mathrm{C} 3-\mathrm{C} 2$ & $177.7(2)$ \\
\hline $\mathrm{N} 1-\mathrm{N} 2-\mathrm{C} 3-\mathrm{C} 5$ & $175.17(18)$ \\
\hline $\mathrm{C} 12-\mathrm{N} 2-\mathrm{C} 3-\mathrm{C} 5$ & $-6.1(3)$ \\
\hline $\mathrm{C} 1-\mathrm{C} 2-\mathrm{C} 3-\mathrm{N} 2$ & $0.7(2)$ \\
\hline $\mathrm{C} 1-\mathrm{C} 2-\mathrm{C} 3-\mathrm{C} 5$ & $-175.3(2)$ \\
\hline $\mathrm{N} 1-\mathrm{C} 1-\mathrm{C} 4-\mathrm{F} 3 \mathrm{~A}$ & $-40.1(6)$ \\
\hline $\mathrm{C} 2-\mathrm{C} 1-\mathrm{C} 4-\mathrm{F} 3 \mathrm{~A}$ & $141.8(6)$ \\
\hline $\mathrm{N1}$ & \\
\hline
\end{tabular}

$\begin{array}{ll}\text { C12-C17-H17A } & 120.4 \\ \text { C16-C17-H17A } & 120.4 \\ \text { O1S-S1S-C1S } & 107.9(2) \\ \text { O1S-S1S-C2S } & 108.1(2) \\ \text { C1S-S1S-C2S } & 98.4(4) \\ \text { S1S-C1S-H1S1 } & 109.5 \\ \text { S1S-C1S-H1S2 } & 109.5 \\ \text { H1S1-C1S-H1S2 } & 109.5 \\ \text { S1S-C1S-H1S3 } & 109.5 \\ \text { H1S1-C1S-H1S3 } & 109.5 \\ \text { H1S2-C1S-H1S3 } & 109.5 \\ \text { S1S-C2S-H2S1 } & 109.5 \\ \text { S1S-C2S-H2S2 } & 109.5 \\ \text { H2S1-C2S-H2S2 } & 109.5 \\ \text { S1S-C2S-H2S3 } & 109.5 \\ \text { H2S1-C2S-H2S3 } & 109.5 \\ \text { H2S2-C2S-H2S3 } & 109.5 \\ \text { O1S-S1SA-C2SA } & 109.7(5) \\ \text { O1S-S1SA-C1SA } & 107.7(4) \\ \text { C2SA-S1SA-C1SA } & 102.1(4) \\ \text { S1SA-C1SA-H1S4 } & 109.5 \\ \text { S1SA-C1SA-H1S5 } & 109.5 \\ \text { H1S4-C1SA-H1S5 } & 109.5 \\ \text { S1SA-C1SA-H1S6 } & 109.5 \\ \text { H1S4-C1SA-H1S6 } & 109.5 \\ \text { H1S5-C1SA-H1S6 } & 109.5 \\ \text { S1SA-C2SA-H2S4 } & 109.5 \\ \text { S1SA-C2SA-H2S5 } & 109.5 \\ \text { H2S4-C2SA-H2S5 } & 109.5 \\ \text { S1SA-C2SA-H2S6 } & 109.5 \\ \text { H2S4-C2SA-H2S6 } & 109.5 \\ \text { H2S5-C2SA-H2S6 } & 109.5 \\ \text { C3-C5-C6-C7 } & \\ \text { C5-C6-C7-C8 } & -177.68(18) \\ \text { C6-C7-C8-C9 } & 17.8(3) \\ \text { C6-C7-C8-C11 } & -0.5(3) \\ \text { C7-C8-C9-C10 } & 179.9(2) \\ \text { C11-C8-C9-C10 } & -0.7(4) \\ \text { C8-C9-C10-C5 } & 178.9(3) \\ \text { C6-C5-C10-C9 } & 0.6(4) \\ \text { C3-C5-C10-C9 } & 0.7(3) \\ \text { N1-N2-C12-C14 } & 176.7(2) \\ \text { C3-N2-C12-C14 } & -53.1(3) \\ \text { N1-N2-C12-C17 } & 128.2(2) \\ \text { C3-N2-C12-C17 } & 124.4(2) \\ \text { C17-C12-C14-C13 } & -54.3(3) \\ \text { N2-C12-C14-C13 } & \\ & \end{array}$




\begin{tabular}{llll}
$\mathrm{C} 2-\mathrm{C} 1-\mathrm{C} 4-\mathrm{F} 1$ & $-36.6(7)$ & $\mathrm{C} 15-\mathrm{C} 13-\mathrm{C} 14-\mathrm{C} 12$ & $0.7(4)$ \\
$\mathrm{N} 1-\mathrm{C} 1-\mathrm{C} 4-\mathrm{F} 1 \mathrm{~A}$ & $86.9(10)$ & $\mathrm{C} 14-\mathrm{C} 13-\mathrm{C} 15-\mathrm{C} 16$ & $-1.0(3)$ \\
$\mathrm{C} 2-\mathrm{C} 1-\mathrm{C} 4-\mathrm{F} 1 \mathrm{~A}$ & $-91.1(10)$ & $\mathrm{C} 14-\mathrm{C} 13-\mathrm{C} 15-\mathrm{S} 1$ & $-179.17(18)$ \\
$\mathrm{N} 1-\mathrm{C} 1-\mathrm{C} 4-\mathrm{F} 2$ & $-95.3(10)$ & $\mathrm{O} 2-\mathrm{S} 1-\mathrm{C} 15-\mathrm{C} 13$ & $144.31(19)$ \\
$\mathrm{C} 2-\mathrm{C} 1-\mathrm{C} 4-\mathrm{F} 2$ & $86.7(10)$ & $\mathrm{O} 1-\mathrm{S} 1-\mathrm{C} 15-\mathrm{C} 13$ & $14.6(2)$ \\
$\mathrm{N} 1-\mathrm{C} 1-\mathrm{C} 4-\mathrm{F} 2 \mathrm{~A}$ & $-155.6(7)$ & $\mathrm{N} 3-\mathrm{S} 1-\mathrm{C} 15-\mathrm{C} 13$ & $-100.59(19)$ \\
$\mathrm{C} 2-\mathrm{C} 1-\mathrm{C} 4-\mathrm{F} 2 \mathrm{~A}$ & $26.4(8)$ & $\mathrm{O} 2-\mathrm{S} 1-\mathrm{C} 15-\mathrm{C} 16$ & $-33.9(2)$ \\
$\mathrm{N} 1-\mathrm{C} 1-\mathrm{C} 4-\mathrm{F} 3$ & $25.0(7)$ & $\mathrm{O} 1-\mathrm{S} 1-\mathrm{C} 15-\mathrm{C} 16$ & $-163.53(17)$ \\
$\mathrm{C} 2-\mathrm{C} 1-\mathrm{C} 4-\mathrm{F} 3$ & $-153.1(7)$ & $\mathrm{N} 3-\mathrm{S} 1-\mathrm{C} 15-\mathrm{C} 16$ & $81.23(19)$ \\
$\mathrm{N} 2-\mathrm{C} 3-\mathrm{C} 5-\mathrm{C} 10$ & $153.3(2)$ & $\mathrm{C} 13-\mathrm{C} 15-\mathrm{C} 16-\mathrm{C} 17$ & $0.7(3)$ \\
$\mathrm{C} 2-\mathrm{C} 3-\mathrm{C} 5-\mathrm{C} 10$ & $-31.4(3)$ & $\mathrm{S} 1-\mathrm{C} 15-\mathrm{C} 16-\mathrm{C} 17$ & $178.82(16)$ \\
$\mathrm{N} 2-\mathrm{C} 3-\mathrm{C} 5-\mathrm{C} 6$ & $-30.8(3)$ & $\mathrm{C} 14-\mathrm{C} 12-\mathrm{C} 17-\mathrm{C} 16$ & $-0.2(3)$ \\
$\mathrm{C} 2-\mathrm{C} 3-\mathrm{C} 5-\mathrm{C} 6$ & $144.4(2)$ & $\mathrm{N} 2-\mathrm{C} 12-\mathrm{C} 17-\mathrm{C} 16$ & $-177.63(18)$ \\
$\mathrm{C} 10-\mathrm{C} 5-\mathrm{C} 6-\mathrm{C} 7$ & $-1.8(3)$ & $\mathrm{C} 15-\mathrm{C} 16-\mathrm{C} 17-\mathrm{C} 12$ & $0.0(3)$ \\
\hline
\end{tabular}

Hydrogen-bond geometry $\left(A,{ }^{\circ}\right)$

\begin{tabular}{lllll}
\hline$D-\mathrm{H} \cdots A$ & $D-\mathrm{H}$ & $\mathrm{H} \cdots A$ & $D \cdots A$ & $D-\mathrm{H} \cdots A$ \\
\hline $\mathrm{N} 3-\mathrm{H} 1 N \cdots \mathrm{O} 1 S$ & $0.87(1)$ & $2.06(1)$ & $2.904(3)$ & $162(2)$ \\
$\mathrm{N} 3-\mathrm{H} 2 N \cdots \mathrm{O} 1 S^{\mathrm{i}}$ & $0.87(1)$ & $2.07(1)$ & $2.930(3)$ & $167(2)$ \\
\hline
\end{tabular}

Symmetry code: (i) $-x+1,-y+1,-z+1$. 